\title{
Modular Typechecking for Hierarchically Extensible Datatypes and Functions
}

\author{
TODD MILLSTEIN \\ University of California, Los Angeles \\ and \\ COLIN BLECKNER and CRAIG CHAMBERS \\ University of Washington
}

One promising approach for adding object-oriented (OO) facilities to functional languages like ML is to generalize the existing datatype and function constructs to be hierarchical and extensible, so that datatype variants simulate classes and function cases simulate methods. This approach allows existing datatypes to be easily extended with both new operations and new variants, resolving a longstanding conflict between the functional and OO styles. However, previous designs based on this approach have been forced to give up modular typechecking, requiring whole-program checks to ensure type safety. We describe Extensible ML (EML), an ML-like language that supports hierarchical, extensible datatypes and functions while preserving purely modular typechecking. To achieve this result, EmL's type system imposes a few requirements on datatype and function extensibility, but EML is still able to express both traditional functional and OO idioms. We have formalized a core version of EML and proven the associated type system sound, and we have developed a prototype interpreter for the language.

Categories and Subject Descriptors: D.3.1 [Programming Languages]: Formal Definitions and Theory-Semantics; syntax; D.3.3 [Programming Languages]: Language Constructs and Features-Classes and objects, data types and structures; procedures, functions, and subroutines

General Terms: Design, Languages, Theory

Additional Key Words and Phrases: Extensible datatypes, extensible functions, modular typechecking

An earlier version of this article appears in the Proceedings of the ACM SIGPLAN International Conference on Functional Programming (ICFP'02), 37:9.

This work was performed while the first author was at the University of Washington. This work was supported in part by NSF grant CCR-9970986, NSF Young Investigator Award CCR-9457767, gifts from Sun Microsystems and IBM, and a Wilma Bradley graduate fellowship.

Authors' addresses: T. Millstein, Computer Science Department, 4531D Boelter Hall, University of California, Los Angeles, Los Angeles, CA 90095-1596; email: todd@cs.ucla.edu; C. Bleckner and C. Chambers, Department of Computer Science and Engineering, University of Washington, Box 352350, Seattle, WA 98195-2350; email: \{colin,chambers\}@cs.washington.edu.

Permission to make digital or hard copies of part or all of this work for personal or classroom use is granted without fee provided that copies are not made or distributed for profit or direct commercial advantage and that copies show this notice on the first page or initial screen of a display along with the full citation. Copyrights for components of this work owned by others than ACM must be honored. Abstracting with credit is permitted. To copy otherwise, to republish, to post on servers, to redistribute to lists, or to use any component of this work in other works requires prior specific permission and/or a fee. Permissions may be requested from Publications Dept., ACM, Inc., 1515 Broadway, New York, NY 10036 USA, fax: +1 (212) 869-0481, or permissions@acm.org. (C) 2004 ACM 0164-0925/04/0900-0836 $\$ 5.00$

ACM Transactions on Programming Languages and Systems, Vol. 26, No. 5, September 2004, Pages 836-889. 


\section{INTRODUCTION}

Many researchers have noted a difference in the extensibility benefits offered by the functional and object-oriented (OO) styles [Reynolds 1978; Cook 1991; Odersky and Wadler 1997; Krishnamurthi et al. 1998; Findler and Flatt 1998; Garrigue 2000; Zenger and Odersky 2001]. Functional languages like ML allow new operations to be easily added to existing datatypes (by adding new fun declarations), without requiring access to existing code. However, new data variants cannot be added without a potentially whole-program modification (since existing functions must be modified in place to handle the new variants). On the other hand, traditional $\mathrm{OO}$ approaches allow new data variants to be easily added to existing class hierarchies (by declaring subclasses with overriding methods), without modifying existing code. However, adding new operations to existing classes requires access to the source code for those classes (since methods cannot be added to existing classes without modifying them in place).

There have been several recent research efforts to integrate the benefits of the functional and OO styles in the context of ML. OCaml [Rémy and Vouillon 1998] adds OO features including class and method definitions to ML. The OO constructs essentially form their own sublanguage that is largely separate from the existing ML datatype and fun constructs. Adding a set of new constructs has the advantage that existing language constructs are minimally affected by the extension, retaining their traditional semantics and typing properties. Further, the augmented language addresses the expressiveness differences of the functional and $\mathrm{OO}$ styles in a very simple way, by providing both options. However, such simplicity comes at a cost to programmers, who are forced to choose up front whether to represent an abstraction with datatypes or with classes. As described above, this decision impacts the kind of extensibility allowable for the abstraction. It may be difficult to determine $a$ priori which kind of extensibility will be required, and it is difficult to change the decision after the fact. Further, it is not possible for the abstraction to enjoy both kinds of extensibility at once.

An alternative approach is to generalize existing ML constructs to support the OO style. OML [Reppy and Riecke 1996], for example, introduces an objtype construct for modeling class hierarchies. This construct can be seen as a generalization of ML datatypes to be hierarchical and extensible. Therefore, programmers need not decide between datatypes and classes up front; both are embodied in the objtype construct. However, OML still maintains a distinction between methods and functions, which have different benefits. New methods may not be added to existing objtypes without modifying existing code, while ordinary ML functions may be. Methods dynamically dispatch on their associated objtype, while functions support ML-style pattern matching.

$\mathrm{ML}_{\leq}$[Bourdoncle and Merz 1997] integrates the OO style further with existing $\mathrm{ML}$ constructs. Like $\mathrm{OML}, \mathrm{ML}_{\leq}$generalizes $\mathrm{ML}$ datatypes to be hierarchical and extensible. Further, methods are simulated via function cases that use OO-style dynamic dispatch semantics. In this approach, programmers need not choose between two forms of extensibility; a single language mechanism 
supports the easy addition of both new operations and new variants to existing datatypes.

However, there are important ways in which $\mathrm{ML}_{\leq}$is not well integrated with existing $\mathrm{ML}$ language features. First, $\mathrm{ML}_{\leq}$does not support $\mathrm{ML}$-style pattern matching. Patterns are essentially restricted to be top-level datatype constructor tests, which are the analogue of dynamic dispatch tests in OO languages. Other common ML-style patterns and patterns on subcomponents cannot be programmed. Second, extensible datatypes are of limited utility without extensible functions, which allow existing functions to be updated with new cases as new data variants are declared. However, $\mathrm{ML}_{\leq}$does not support extensible functions: all function cases are provided when a function is declared. The authors sketch a source-level language that supports extensible functions. Unfortunately, this critical generalization of their work causes a loss of modular reasoning: static typechecking of a program cannot be completed until link time, when all modules are available. Therefore, important software engineering benefits are lost, including early detection of errors, libraries that are guaranteed to be typesafe in any context satisfying their interface requirements, independent development of typesafe modules by separate teams of programmers, and incremental modification (and subsequent incremental retypechecking) of code.

The checks that must be delayed to link time in $\mathrm{ML}_{\leq}$constitute implementation-side typechecking (ITC) [Chambers and Leavens 1995], which ensures that each function in the program is exhaustively and unambiguously implemented. Implementation-side typechecking contrasts with client-side typechecking of functions, which checks that each function application in the program is type-correct. Client-side typechecking in $\mathrm{ML}_{\leq}$is standard and can be performed modularly.

In traditional functional languages, ITC checks each function for match nonexhaustive and match redundant errors. Each function can be checked modularly, since a function declaration includes all of its cases, and datatypes are not extensible. In traditional OO languages, ITC checks that each class declares or inherits a most-specific method for each supported operation. Each class can be checked modularly, since a class declaration includes all of its (noninherited) methods and new operations cannot be added to existing classes.

The implicit restrictions in the traditional functional and $\mathrm{OO}$ settings that allow for modular ITC do not hold in the presence of extensible datatypes and functions. Unlike traditional functional languages, no module is guaranteed to have access to all of a function's cases. Unlike traditional OO languages, no module is guaranteed to have access to all of a datatype variant's associated functions and function cases. Therefore, $\mathrm{ML}_{\leq}$is forced to perform ITC globally, when the whole program is available.

In this work, we describe an ML-like language called Extensible $\mathrm{ML}^{1}$ (EML). EML introduces a class construct, which is a form of hierarchical, extensible datatype in the spirit of the constructs in $\mathrm{OML}$ and $\mathrm{ML}_{\leq}$. As in $\mathrm{ML}_{\leq}$, methods

${ }^{1}$ Not to be confused with Extended ML [Kahrs et al. 1997]. 
are simulated by function cases. In addition:

- EmL generalizes the $\mathrm{OO}$ dispatching semantics in $\mathrm{ML}_{\leq}$to allow arbitrary MLstyle patterns. This generalization provides idioms that are not expressible by either traditional functional or traditional OO languages.

- EmL supports extensible functions while preserving purely modular typechecking: each module can be typechecked given only the interfaces of the modules it statically depends upon (in a sense described later), with no wholeprogram checks required. To make per-module implementation-side typechecking sound without necessitating link-time checks, EML's type system imposes certain requirements via the notion of a function's owner position, which serves to coordinate otherwise independent extensions to the function. The owner position is inspired by the properties of a method's receiver that achieve modular typechecking in traditional OO languages. Despite the imposed requirements, EmL's classes and functions are still able to simultaneously express traditional functional and $\mathrm{OO}$ extensibility idioms. The requirements are adapted from our earlier work on Dubious [Millstein and Chambers 1999, 2002], a calculus designed to explore modular typechecking for OO languages based on multimethods.

The rest of the paper is organized as follows. Section 2 describes EmL by example. Section 3 discusses the challenges for performing modular implementationside typechecking in EML and presents our solution to these challenges. Section 4 defines Mini-EmL, a core language for EmL used to formalize our modular type system. Section 5 describes challenges for the interaction of EML's constructs with an ML-style module system, including signature ascription and functors. Section 6 discusses related work, and section 7 concludes. The appendix contains the type soundness proof for Mini-EmL.

\section{EML BY EXAMPLE}

Figure 1 shows an EML implementation of integer sets. Classes, functions, and function cases are declared in ML-style structs. In our discussion we assume that structs contain only EmL's new declarations. This assumption is lifted in Section 5, which describes the interaction of EmL's features with an ML-style module system.

\subsection{Classes}

The Set class in Figure 1 is the top of the integer set hierarchy. The ListSet class inherits from Set, implementing sets via lists. The CListSet class inherits from ListSet, additionally keeping track of the number of elements in the set. A program's subclass relation is the reflexive, transitive closure of the declared extends relation. The Set class is declared abstract, so it may not be instantiated, while its subclasses ListSet and CListSet are concrete.

Each class declares a record type of its instance variables, using the of clause. Superclass instance variables are inherited: the representation type of a class $C$ is the representation type (recursively) of its direct superclass (if any) 


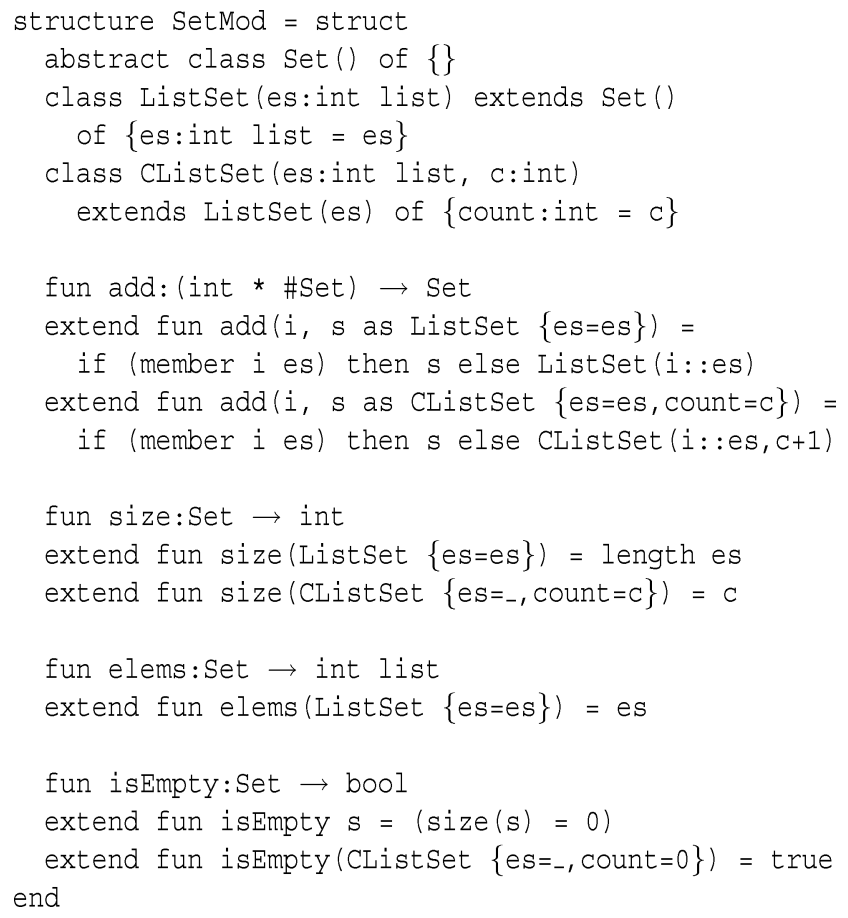

Fig. 1. A hierarchy of integer sets in EmL.

concatenated with the type in the of clause in $C$ 's declaration. For example, the representation type of CListSet is \{es:int list, count:int\}, since ListSet's representation type is $\{e s:$ int list\}.

Each class declaration also implicitly declares a constructor, similar to constructor declarations in OCaml [Rémy and Vouillon 1998] and XMOC [Fisher and Reppy 2000], a core language for Moby [Fisher and Reppy 1999]. For example, the CListSet constructor expects arguments es of type int list and $c$ of type int, initializes inherited instance variables via the call ListSet(es) to the superclass constructor, and initializes the new count instance variable to c. In general, the arguments to the superclass constructor call and the instance-variable initializers may be arbitrary expressions. It would be straightforward to allow a class to have multiple constructors by introducing a separate constructor declaration, similar to "makers" in Moby.

Classes are as expressive as ordinary ML-style datatypes. An ML datatype of the form

datatype $\mathrm{DT}=\mathrm{C}_{1}$ of $\left\{\mathrm{L}_{11}: \mathrm{T}_{11}, \ldots, \mathrm{L}_{1 m}: \mathrm{T}_{1 m}\right\}|\cdots| \mathrm{C}_{r}$ of $\left\{\mathrm{L}_{r 1}: \mathrm{T}_{r 1}, \ldots, \mathrm{L}_{r n}: \mathrm{T}_{r n}\right\}$

is encoded in EmL by the following class declarations:

abstract class DT of \{\}

class $\mathrm{C}_{1}\left(\mathrm{I}_{11}: \mathrm{T}_{11}, \ldots, \mathrm{I}_{1 m}: \mathrm{T}_{1 m}\right)$ extends $\mathrm{DT}()$ of $\left\{\mathrm{L}_{11}: \mathrm{T}_{11}=\mathrm{I}_{11}, \ldots, \mathrm{L}_{1 m}: \mathrm{T}_{1 m}=\mathrm{I}_{1 m}\right\}$

ACM Transactions on Programming Languages and Systems, Vol. 26, No. 5, September 2004. 
..

class $\mathrm{C}_{r}\left(\mathrm{I}_{r 1}: \mathrm{T}_{r 1}, \ldots, \mathrm{I}_{r n}: \mathrm{T}_{r n}\right)$ extends $\mathrm{DT}()$ of $\left\{\mathrm{L}_{r 1}: \mathrm{T}_{r 1}=\mathrm{I}_{r 1}, \ldots, \mathrm{L}_{r n}: \mathrm{T}_{r n}=\mathrm{I}_{r n}\right\}$

For example, the declarations of the Set and ListSet classes in Figure 1 could have been written equivalently as follows:

datatype Set $=$ ListSet of $\{$ es:int list $\}$

Classes additionally generalize ML-style datatypes to be extensible, whereby new variants can be written in modules other than the one declaring the datatype, and hierarchical, whereby variants can have their own "subvariants." The CListSet subclass of ListSet in Figure 1 illustrates class hierarchies; datatype extensibility is described in Section 2.4 below. In addition to being extensible and hierarchical, classes are also full-fledged types while ML variants are not. For example, classes can appear in a function's argument or return type.

A concrete class is instantiated by invoking its constructor. For example, the result of ListSet $([5,3])$ is an instance of ListSet representing the set $\{5,3\}$. Like values of ML datatypes, class instances have no special object identity or mutable state; refs can be used in a class's representation type for this purpose.

Classes support only single inheritance. Single inheritance of classes is compatible with the ML style, in which each data variant conceptually singly inherits from the corresponding datatype, as shown in the above encoding of datatypes into classes. However, EML can support multiple interface inheritance, like Java [Arnold et al. 2000; Gosling et al. 2000]. Interfaces in EML have a syntax similar to classes, but they do not have instance variables and do not declare a constructor. For example, the abstract Set class in Figure 1 could instead be declared to be an interface as follows:

interface Set

Classes may be declared to implement interfaces. Given the above declaration, the declaration of ListSet would be modified to look as follows:

class ListSet(es:int list) implements Set of $\{$ es:int list $=$ elems $\}$

Like abstract classes, interfaces may not be instantiated. Unlike (abstract or concrete) classes, an interface can inherit from multiple interfaces via its extends clause, and a class can inherit from multiple interfaces via its implements clause. As described below, interfaces must obey additional constraints in exchange for this extra expressiveness.

\subsection{Functions and Function Cases}

To make functions extensible, we break an ML-style function declaration into two pieces. The fun declaration introduces a function and specifies its type. The add function in Figure 1, for example, is declared to accept one integer and one instance of Set or a subclass, and to return an instance of Set or a subclass. The \# in the function's argument type signifies that the second argument to add is in the owner position. Every EmL function declaration must specify exactly one owner position, and the type at that position must be a class or interface. 
The owner position can be arbitrarily nested within a function's argument type. As a syntactic sugar, if a function's entire argument type is simply a class or interface, then that sole argument position is assumed to be the owner. This sugar is used in the declarations of the other functions in Figure 1. A function and its cases must satisfy several requirements with respect to its owner position, to ensure that the function can be modularly checked for exhaustiveness and unambiguity. These requirements are discussed in Section 3. The owner position has no dynamic effect.

The extend fun declaration adds a case to an existing function. The declaration specifies the name of the function being extended, a pattern guard, and the new case's body. There are two size function cases in Figure 1, handling ListSets and CListSets, respectively. In a traditional OO language, these cases would be declared as size methods in the ListSet and CListSet class declarations.

The extend fun declaration updates the set of cases associated with the specified function, instead of creating a new function containing the extra case. The chosen semantics is necessary for extensible functions to faithfully model OO-style methods. For example, suppose a new structure HashSetMod creates a Set subclass HashSet, which represents sets using hash tables, along with an overriding size function case for HashSet. (Such a structure is described in Section 2.4 below.) The semantics of extend fun allows HashSet's size case to be invoked from the existing size application in the first isEmpty case in Figure 1, even though HashSet and its size case are not known in SetMod. Similarly, clients of the set hierarchy need not be aware of all set subclasses and need not be modified as new Set subclasses are introduced.

A regular ML-style function consisting of $n$ function cases is encoded in EML as a fun declaration followed by $n$ extend fun declarations. EmL functions can be passed to and returned from other functions, like lambdas and ML-style functions. However, a function's extensibility is second-class: new cases may only be added to statically known functions.

Patterns in EML allow the expression of both OO-style dynamic dispatch and functional-style patterns. OO dispatch is encoded by a class pattern. For example, the second size case is only applicable dynamically if the argument is an instance of CListSet or a subclass. Class patterns contain a representation pattern, which supports dispatch on instance variables recursively. While the second size case's representation pattern matches the representation of any instance of CListSet (or a subclass), the second isEmpty case requires the argument's count instance variable to have the value zero. As in ML, a pattern may bind identifiers for use in its case's body. For example, the first add case binds the identifiers i, s, and es.

An OO-style "best-match" policy decides which function case to invoke; their order does not matter. Given an application of function $f$ with argument value $v$, first the applicable cases of $f$ for $v$ are retrieved. These are the cases that have a pattern that $v$ matches. Of the applicable cases, the unique case that is more specific than all other applicable cases is invoked. Intuitively, case $c_{1}$ is more specific than case $c_{2}$ if the set of values matching $c_{1}$ 's pattern is a subset of the set of values matching $c_{2}$ 's pattern. We call the invoked case the 
most-specific applicable case. If a function application has no applicable cases, a match nonexhaustive error occurs. If a function application has at least one applicable case but no most-specific one, a match ambiguous error occurs.

For example, consider the invocation isEmpty(CListSet $([], 0))$. Both isEmpty cases in Figure 1 are applicable to the argument value, and the second case is invoked because it is the more-specific one. The best-match semantics contrasts with the traditional "first-match" semantics of function cases in ML. The first-match semantics does not generalize naturally to handle extensible datatypes and functions, where typically the more-specific function cases are written after the less-specific ones, as new data variants are defined.

Implementation-side typechecking of a function ensures that match nonexhaustive and match ambiguous errors cannot occur at run time. Conceptually this checking entails ensuring that each type-correct argument to the function has a most-specific applicable case to invoke. Each module's typechecks include ITC for functions whose exhaustiveness and unambiguity may be affected by the module. These are functions declared in the module, functions with cases declared in the module, and functions that can accept instances of classes declared in the module. For example, ITC of SetMod in Figure 1 checks the four functions declared there. Consider checking the size function for exhaustiveness and unambiguity. Any ListSet instance will invoke the first size case, and any CListSet instance will invoke the second size case. The Set class need not have a most-specific applicable case, because set is declared abstract. Therefore, ITC for size succeeds. On the other hand, if the first size case were missing, a match nonexhaustive error would be statically signaled. Alternatively, if another size case with pattern ListSet \{es=es\} were declared, a match ambiguous error would be statically signaled.

Unlike (both concrete and abstract) classes, interfaces may not appear in patterns. This restriction is the EmL analogue of Java's restriction that an interface have no concrete methods. Both restrictions remove the potential for dynamic-dispatch ambiguities caused by multiple inheritance. Because of EmL's restriction, interfaces do not impact ITC any differently from abstract classes. Therefore we ignore interfaces in the remainder of the paper.

\subsection{Adding New Functions}

As with ML datatypes, but unlike traditional classes, EmL supports the easy addition of new functions to an existing class hierarchy. For example, Figure 2 adds a function for computing the union of two Sets, without modifying any code in the SetMod module. ${ }^{2}$ Two union function cases are provided. The first case is applicable to any pair of Sets. The second union case provides a more efficient implementation for two ListSets. ITC of UnionMod checks union for exhaustiveness and unambiguity. Any pair consisting of ListSet and/or CListSet instances will invoke the second union case, so the function's check succeeds.

${ }^{2}$ Technically, all references to Set, ListSet, add, and elems in UnionMod should instead be to SetMod.Set, SetMod.ListSet, SetMod.add, and SetMod.elems. For readability, we omit the full path names in examples when clear from context. 


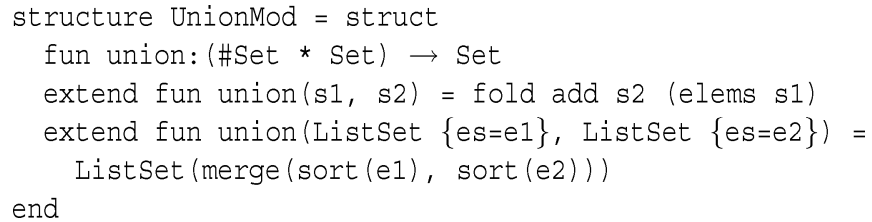

Fig. 2. Adding new functions in EML.

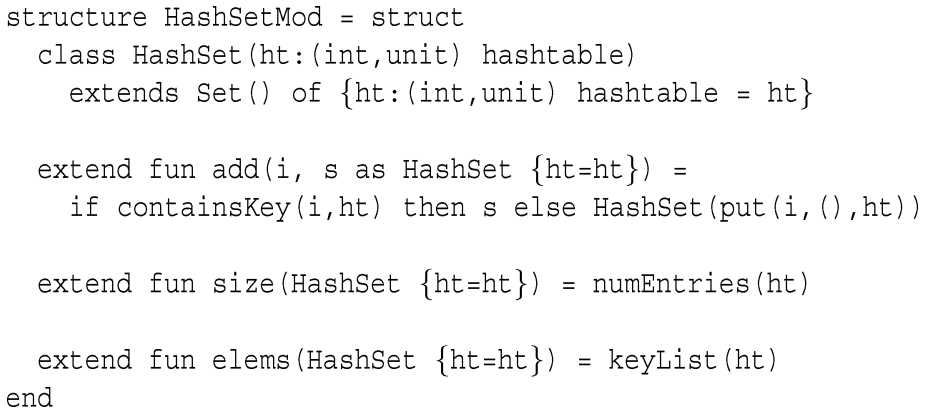

Fig. 3. Adding new data variants in EmL.

\subsection{Adding New Data Variants}

Unlike ML datatypes, classes in EmL also support the easy addition of new data variants to existing hierarchies, without modifying existing code. An example is shown in Figure 3, which provides a new implementation HashSet of sets using an existing implementation (not shown) of hash tables. Implementations of add, size, and elems are provided for the new kind of set, while the first isEmpty case in Figure 1 is inherited. In a traditional OO language, HashSetMod corresponds to the declaration of a new subclass of Set with some overriding methods. ITC of HashSetMod rechecks the four functions declared in Figure 1 to ensure that they handle HashSet instances properly. For example, if the new size case were not declared, a match nonexhaustive error for size would be signaled statically.

UnionMod and HashSetMod from Figures 2 and 3 illustrate EML's support for both functional and $\mathrm{OO}$ forms of extensibility in a single class hierarchy. The original Set abstraction is flexibly reused by clients, who augment the abstraction with client-specific functionality and also add a specialized implementation (subclass) of the abstraction, all without modifying existing code. UnionMod and HashSetMod are completely independent: either, both, or neither module could be linked into the final program. In this way, different versions of the Set abstraction may be used in different programs, depending on the needs of each application.

If both UnionMod and HashSetMod are present in a program, then HashSet implicitly supports the union operation and inherits any applicable cases. This expressiveness is at the heart of the problem of modular ITC. Because the two modules are independent, neither is "aware" of the other during its static 


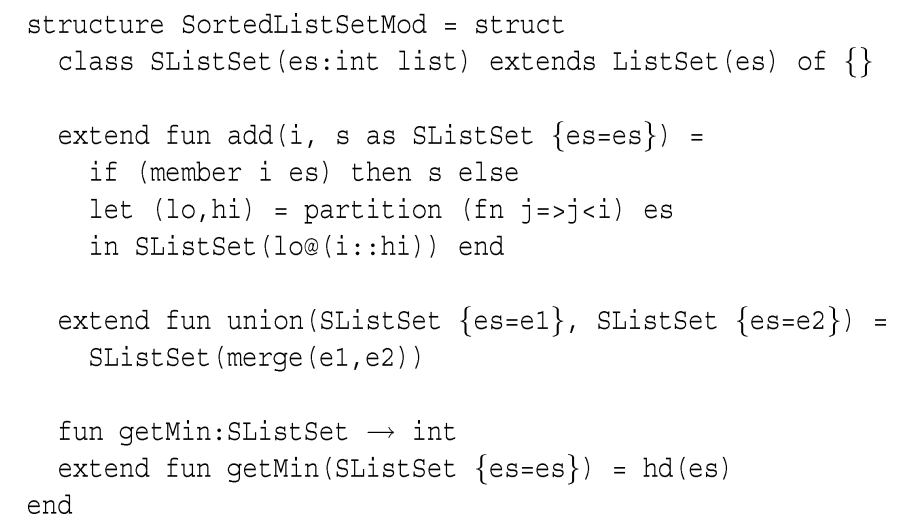

Fig. 4. Class hierarchies in EML.

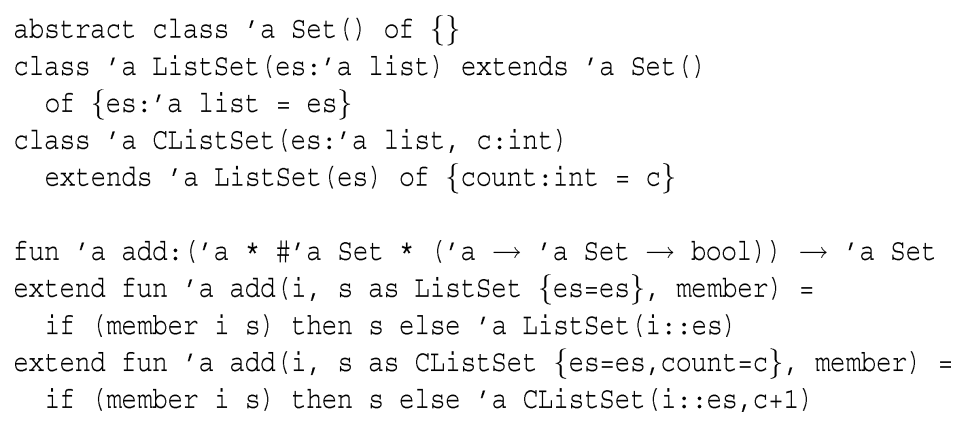

Fig. 5. Polymorphic sets in EmL.

typechecks. Therefore, neither module's ITC ensures that union is exhaustively and unambiguously implemented for HashSets. In this example, union happens to have a case that handles HashSets (by handling any pair of sets). Without extra requirements, however, things do not always work out so well, as we show in Section 3.

Another example of data-variant extensibility is illustrated in Figure 4. A new subclass of ListSet is created, representing an implementation of sets via sorted lists. Overriding cases of add and union are provided, as well as a new operation for accessing the minimum element of a set implemented as a sorted list. ITC of SortedListSetMod checks the four functions declared in Figure 1 as well as the new getMin function to ensure exhaustiveness and unambiguity for SListSets.

\subsection{Parametric Polymorphism}

EML supports a polymorphic type system. Class, function, and function case declarations optionally bind type variables. References to a polymorphic class or function specify a particular type instantiation. As an example, Figure 5 shows some of the declarations for a polymorphic version of the sets in Figure 1. Each class in the set hierarchy is now parameterized by the element type, as is the add function. As a convenience, each function case is also explicitly parameterized, 
allowing its function's type variables to be renamed for use in the case's body. References to classes in a case's pattern do not contain type parameters. The unique typesafe instantiation for such classes is determined from the declared argument type (for example, the reference to CListSet in the second add case's pattern is implicitly'a CListSet).

EML's polymorphic type system is deliberately simple. First, subclasses must have the same type variables as their superclasses. This requirement is consistent with polymorphism in ML, where data variants have the same type variables as their associated datatype. Also, type parameters are invariant; for example, $\mathrm{T}_{1}$ ListSet is a subtype of $\mathrm{T}_{2}$ Set if and only if $\mathrm{T}_{1}=\mathrm{T}_{2}$. Finally, there is no support for bounded polymorphism [Cardelli and Wegner 1985]. We have chosen to make the polymorphic type system simple because polymorphism is orthogonal to the problems of modular ITC that we address in this work. Those problems arise from the fact that some related classes, functions, and function cases are not modularly "aware" of one another; the problems are neither reduced nor exacerbated by polymorphic types. Therefore, EmL's polymorphic type system could be generalized in standard ways without affecting modular typechecking. For example, EmL could adopt $\mathrm{ML}_{\leq}$'s subtype-constrained polymorphic type system for extensible datatypes and functions [Bourdoncle and Merz 1997].

EmL is also explicitly typed. This contrasts with ML's polymorphic type system, which supports type inference. Unfortunately, supporting both subtyping and polymorphic type inference is known to be difficult [Fuh and Mishra 1990; Hoang and Mitchell 1995; Nordlander 1999]. It would be useful to explore forms of local type inference [Pierce and Turner 2000] to ease the type-annotation burden somewhat. Recent work of Bonniot Bonniot [2002] has presented a simplified account of $\mathrm{ML}_{\leq}$'s type system and shown how to incorporate a form of local type inference, so this could be a promising foundation for augmenting EML.

\section{MODULAR IMPLEMENTATION-SIDE TYPECHECKING}

This section focuses on the problem of modular ITC for EML. First we define our notion of modular typechecking. Next we illustrate the ways in which straightforward modular ITC is unsound. Finally we describe the requirements we impose to achieve modular type safety.

\subsection{Modular Typechecking}

We say that a language's typechecking scheme is modular if it has two properties. First, each module $m$ can be typechecked given only the signatures ${ }^{3}$ of other modules (without requiring access to the associated implementations). Second, $m$ can be typechecked given only those signatures that $m$ statically depends upon. Module $m$ statically depends upon signature $s$ if either of the

\footnotetext{
${ }^{3}$ We employ the ML terminology signature, rather than the more generic interface, to avoid confusion with the notion of interface in languages like Java and EML.
} 
following conditions holds:

- Module $m$ refers to a name that is bound in $s$.

- Module $m$ statically depends upon module signature $s^{\prime}$, and $s^{\prime}$ refers to a name that is bound in $s$.

Traditional functional languages can support modular typechecking. For example, each structure in ML could be typechecked given only its statically depended-upon signatures. A structure's signature is either an explicitly ascribed one or else the structure's principal signature. Similarly, each class in a standard OO language can be typechecked given only the statically dependedupon class signatures. Informally, the signature of a class consists of its list of superclasses and superinterfaces, the types of its visible fields, and the headers, but not bodies, of its visible methods. Signatures for modular typechecking in the context of Java have been formally defined by others [Drossopoulou et al. 1999; Ancona et al. 2002].

A modular typechecking scheme for EmL must typecheck each structure given only the signatures it statically depends upon. An EML signature contains a sequence of class, function, and function-case specifications. The syntax of each specification is identical to that of its associated declaration, but with all expressions removed. In particular, each class specification does not include the arguments to the superclass constructor or the instance-variable initializer expressions, and each extend fun specification does not include the case's body.

For now, EmL does not support explicit signature ascription; this ability is discussed in Section 5. Instead, each structure is implicitly ascribed to its principal signature. This signature has the same name as its associated structure and contains a specification for each declaration in the structure. Further, each specification is identical to its associated declaration, but with all expressions removed. For example, Figure 6 shows the principal signature of SetMod from Figure 1.

Classes, functions, and cases that are declared in a module $m$ or specified in a signature upon which $m$ statically depends are said to be available during the typechecking of $m$. All other classes, functions, and cases are unavailable and may not be considered during the typechecking of $m$. Our definition of modular typechecking validates the intuition that union of Figure 2 and HashSet of Figure 3 are not "aware" of one another. Neither UnionMod nor HashSetMod statically depends upon the other's signature. Therefore, HashSet is unavailable during modular typechecks on UnionMod and union is unavailable during modular typechecks on HashSetMod, so neither module's typechecks ensure that union properly handles HashSets.

One undesirable feature of an EML signature is the fact that it reveals information about a function's cases. Ideally, clients of an EML structure could be safely typechecked given only type information about the classes and functions declared in a module, without requiring any knowledge of individual extend fun declarations. Such a revised EmL signature would adhere to Cardelli's notion of modular typechecking, which requires each structure to be typechecked given only the types of its free variables [Cardelli 1997]. However, 


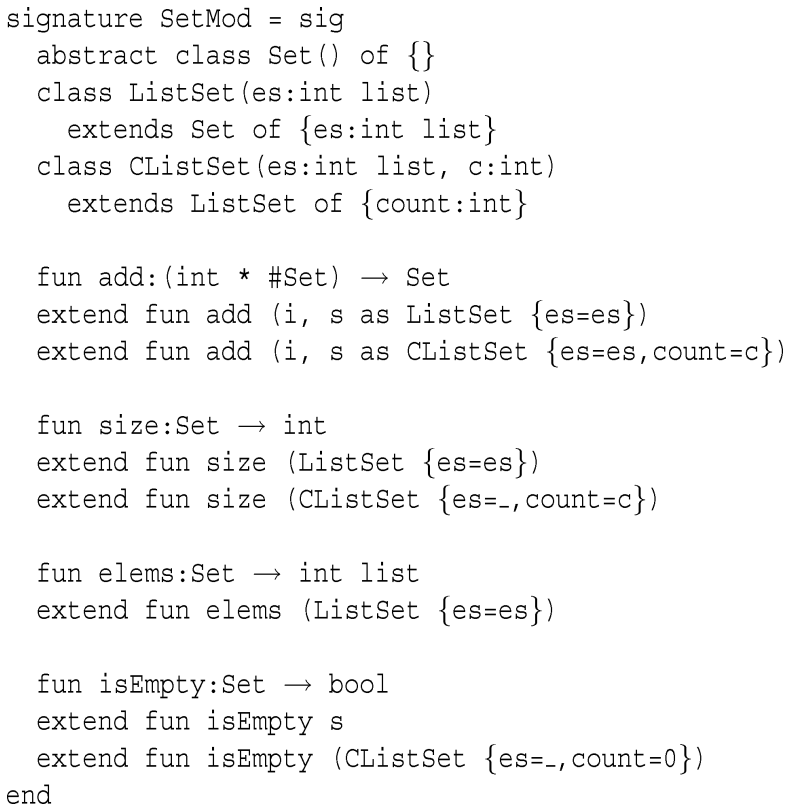

Fig. 6. The principal signature of SetMod.

EML's expressiveness makes this stronger notion of modularity infeasible. A client of a structure may need access to the patterns in a structure's extend fun declarations in order to ensure that the associated functions remain exhaustive and unambiguous in the face of the client's declared classes and function cases. Importantly, clients need never have access to the bodies of those extend fun declarations.

EML is not alone in suffering from this problem. Indeed, EML's notion of modularity generalizes the notion of modularity in traditional OO languages, which also does not meet Cardelli's definition. As mentioned above, the signature for a class in such languages includes the headers of individual methods. Importantly, the signature for a class $C$ reveals which of the operations that $C$ supports are implemented by a method that dynamically dispatches on $C$ at the receiver; methods for other operations are inherited from superclasses. This information about individual methods is necessary so that subclasses can ensure the exhaustiveness and unambiguity of all operations they support. For example, an abstract class's concrete method headers determine which operations must be implemented by concrete subclasses and which need not be. As another example, a class that inherits from multiple other classes requires knowledge of the method implementations in those classes in order to detect potential ambiguities. Revealing the patterns of function cases is the EML generalization of this revealing of individual methods' receivers in traditional OO languages.

\subsection{Implementation-Side Typechecking and Modularity}

Consider ITC for an EML module $m$. A straightforward approach to modular ITC checks each of $m$ 's available functions $f$ for exhaustiveness and unambiguity, 


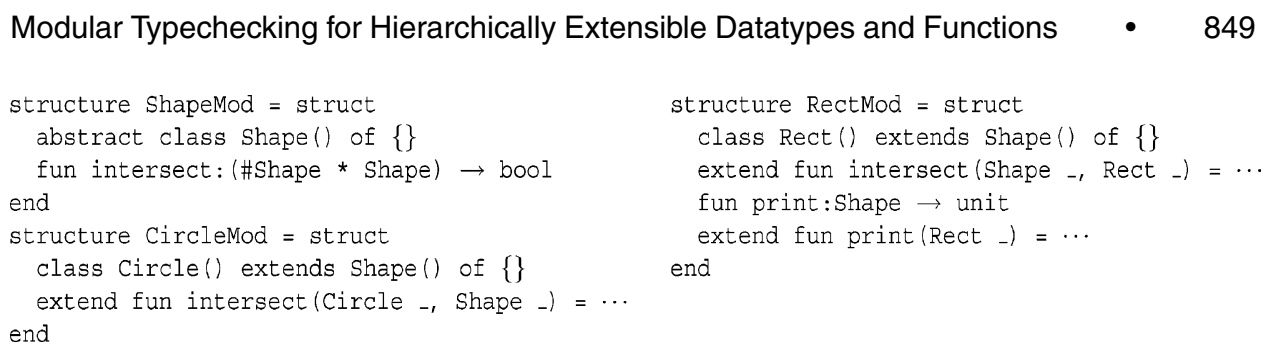

Fig. 7. Challenges for modular implementation-side typechecking.

given all available function cases and classes. We call this approach naive modular ITC. Unfortunately, naive modular ITC is unsound. The hierarchy of EML classes in Figure 7 illustrates the kinds of problems that can occur. Naive modular ITC in ShapeMod checks intersect for exhaustiveness and unambiguity. Since ShapeMod doesn't statically depend upon any signatures (other than its own), the check succeeds vacuously: Shape is abstract and so need not have an intersect implementation. Since CircleMod declares a new intersect case, intersect is again checked during naive modular ITC in CircleMod. CircleMod statically depends on the signature of ShapeMod but not that of RectMod, so CircleMod's check does not consider the Rect class. ${ }^{4}$ Therefore, the only argument to check from CircleMod is a pair of two Circles. The intersect case in CircleMod is most specific for two Circles, so intersect is found to be exhaustive and unambiguous. By similar reasoning, intersect passes the checks from RectMod, since RectMod does not statically depend on the signature of CircleMod.

Therefore each module's naive modular ITC declares the intersect function to be both exhaustive and unambiguous. However, intersect has neither of these properties. If intersect is invoked on a pair of a Rect and a Circle (in that order), a match nonexhaustive error will occur since neither intersect case is applicable. If intersect is invoked on a pair of a Circle and a Rect (in that order), a match ambiguous error will occur since both intersect cases apply but neither is more specific than the other.

A final problem concerns the print function in RectMod. Since RectMod does not statically depend on CircleMod's signature, RectMod's naive modular ITC finds print to be exhaustive and unambiguous. However, if a Circle is ever passed to print, a match nonexhaustive error will result.

\subsection{Achieving Modular ITC}

As we have seen, naive modular ITC is too permissive, allowing forms of extensibility that are not modularly typesafe. To address this problem, we impose some additional requirements on EML modules that ensure the soundness of modular ITC. This section informally describes EML's modular ITC algorithms and requirements. ITC is divided into checks for exhaustiveness and checks for unambiguity; we discuss each in turn.

A fundamental design goal for EML's modular type system is that it still allow the use of both functional and $\mathrm{OO}$ extensibility idioms in a single class hierarchy. We are willing to sacrifice other kinds of extensibility expressible with

${ }^{4}$ Indeed, RectMod may not even have been written when CircleMod is typechecked. 
extensible datatypes and functions in order to support the traditional functional and $\mathrm{OO}$ idioms in a modularly typesafe manner. Functional languages allow a new function to be added to an existing datatype. Therefore, EML must allow a new function to be added to an existing class. OO languages allow a new subclass to be added to an existing class, along with overriding methods that have the new subclass as their receiver. To formulate this idiom in EML we employ a function's owner position, which generalizes a similar notion in the Dubious language [Millstein and Chambers 2002]. The type at the owner position in a function's argument type is the function's owner. For example, Set is the owner of add in Figure 1. To express the OO extensibility idiom in EML, we must allow a new subclass to be added to an existing class $C$, along with overriding cases of functions for which $C$ is the owner.

3.3.1 Modular Exhaustiveness Checking. For the purposes of modular exhaustiveness checking, we partition functions into two categories. A function is called internal if it is declared in the same module as its owner; otherwise the function is external. An internal function is guaranteed to be available to all modules that declare subclasses of the function's owner, while that is not true of an external function. Therefore, an internal function can be thought of as part of the "initial signature" of its owner class, while an external function is a later extension to that signature. External functions have no analogue in traditional OO languages, in which a class's methods must all be declared with the class. Modular exhaustiveness checking consists in enforcing two requirements, one for external functions and the other for internal functions, which are discussed in turn.

Exhaustiveness Requirement for External Functions. Consider the exhaustiveness problem with the print function in RectMod of Figure 7. Because new subclasses can be added to existing classes, some subclasses of a function's owner may not be available in the function's module. Indeed, Circle is not available in print's module. On the other hand, because print is external, there is no guarantee that print will be available to all modules declaring subclasses of Shape. Indeed, print is not available to Circle's module. Therefore, to modularly ensure that print is exhaustive, we require its module to contain a global default case. A global default is a case whose pattern is applicable to all type-correct arguments to the function. In general, we require each module that declares an external function to include a global default case for the function.

Therefore, ITC on RectMod fails, because the global-default requirement is not satisfied for its external function print. If print had a case with the pattern (Shape \{\} ), for example, then the requirement would be satisfied and the exhaustiveness problem for Circle would be avoided. As another example, the external function union in Figure 2 satisfies the requirement because its first case is a global default, thereby handling the unavailable HashSet class of Figure 3 and any other unavailable Set subclasses.

The ability to write an appropriate global default case depends heavily on the functionality available in the "initial signature" of a class. In particular, the global-default requirement only works well for external functions whose behavior can be expressed solely in terms of that signature. For example, the 
requirement is very natural for union in Figure 2: the given global default is appropriate for all unseen Set subclasses. However, had SetMod in Figure 1 not included the elems function, it is unlikely that a reasonable default case for union could be written. Instead, the implementer may have no choice but to make the global default case simply throw an exception, which is not much different from the run-time match nonexhaustive error being prevented by the global-default requirement.

The global-default requirement does not impose an extra burden from the point of view of standard OO languages, as such languages do not even allow external functions to be declared. However, standard functional languages like ML do allow external functions, without requiring global default cases. Those languages disallow data-variant extension, so an external function can be modularly checked against all possible data variants. In contrast, EML's modular ITC must always allow for the possibility of unavailable subclasses of a function's owner. Section 5 introduces a mechanism for sealing class hierarchies, which can be used to obtain the semantics of ordinary (nonextensible) datatypes in EML. Analogous with ML, external functions on a sealed class hierarchy need not include a global default case.

Exhaustiveness Requirement for Internal Functions. Consider the exhaustiveness problem for a pair of one Rect and one Circle in the internal intersect function of Figure 7. One way to solve the problem would be to require a global default case, as we require for external functions. Indeed, if ShapeMod contained an intersect case that is applicable to any pair of Shapes, the problem would be resolved. While requiring global default cases solves the problem, it is unnecessarily burdensome. As mentioned earlier, an internal function is guaranteed to be available to all modules declaring subclasses of the function's owner. Therefore, rather than requiring the function's module to handle all unknown subclasses, we can require each module that declares a concrete subclass of the function's owner to ensure exhaustiveness for its subclass. This idea is inspired by standard OO languages, in which a method in an abstract class may safely remain unimplemented, with each concrete subclass declaring or inheriting a concrete implementation of the method.

Our requirement is that each module that declares a concrete subclass $C$ of an internal function's owner must also declare or inherit a local default case for the function. A local default case is one whose pattern accepts instances of $C$ and subclasses at the owner position, while every other argument position can be passed any value of the appropriate type. Local default cases are the EML analogue of traditional $\mathrm{OO}$ methods, which dispatch on the surrounding class at the receiver position and do not dispatch on any other argument position. A class's local default cases ensure that the class exhaustively implements all of the functions in its "initial" signature.

Given the local-default requirement, ITC on RectMod fails to typecheck because it does not declare or inherit a local default intersect case for Rect. (An isomorphic error would occur in CircleMod if the second argument position in the pair were designated the owner position.) A local default case is required regardless of what other intersect cases exist, like the one shown in RectMod. The 
requirement would be satisfied, for example, if RectMod included an intersect case with pattern (Rect _, Shape _), accepting Rects at the owner position and accepting all Shapes in the other position. That case resolves the exhaustiveness problem for a pair of one Rect and one Circle. A global default case need not be written: intersect may still be safely left unimplemented for two Shapes. As another example, the internal add function in Figure 1 does not have a global default case. Instead, it has local default cases for the two concrete Set subclasses. When HashSet is introduced in Figure 3, an associated local default for add is declared, satisfying the requirement and ensuring that add is exhaustive for HashSets.

The local-default requirement does not impose an extra burden from the point of view of standard OO languages. Whenever a local default case of some internal function $f$ is required for a class $C$, an OO language would require $C$ 's declaration to contain an $f$ method, so that $C$ is properly implemented. Therefore, the abstract-class idioms of traditional OO languages are preserved in EmL. However, standard functional languages do allow internal functions, without requiring local default cases. As above, this is possible because such languages disallow data-variant extension. In contrast, EML's ITC must always assume the possibility of unavailable concrete subclasses of classes in nonowner positions of a function's argument type. Again, we can use sealing, discussed in Section 5, to remove the burden of writing local default cases.

Precise Default Checking. One practical issue that arises with checking for (global and local) defaults in EML is the need to avoid being overly conservative. For example, a simple way to enforce the global-default requirement is to check that every external function has a case whose pattern is the wildcard pattern (_). While using this algorithm in EML would be safe, it would also cause many exhaustive functions to nonetheless be rejected. For example, the union function in Figure 2 has a global default case, but it fails the above check. To remedy this imprecision, EML instead checks that each external function has a case whose pattern is some global default pattern: any argument of the function's type matches the pattern. In the case of union, there are several possible global default patterns, including ${ }_{-},\left(_{-},-\right),\left(\right.$s1, s2), (Set _ , ${ }_{-}$), and (Set \{\} , Set _).

EML performs the revised check by generating a precise global default pattern and then requiring that this pattern be at least as specific as some case's pattern; that case is a global default. The algorithm retains precision via the notion of a pattern's depth, which is essentially the height of the pattern's abstract syntax tree representation. A valid global default case will not be overlooked by the algorithm as long as the generated global default pattern has a depth no smaller than that of the case's pattern. Therefore, EML generates the pattern to have a depth equal to the maximum depth of any available case on the function. ${ }^{5}$ In the union example, exhaustiveness checking generates the global default pattern (Set \{\} , Set \{\} ), matching the depth of the second case's pattern. The check

\footnotetext{
${ }^{5}$ Because class patterns allow pattern matching on a class's representation, which may recursively involve more class patterns, there is in general no a priori maximal depth for the patterns of a given function.
}

ACM Transactions on Programming Languages and Systems, Vol. 26, No. 5, September 2004. 
succeeds because the generated pattern is at least as specific as the first case's pattern, so that case is a valid global default. A similar algorithm is used for checking for local default cases. These algorithms are implemented in the EML prototype interpreter and are formalized in Section 4.

\subsubsection{Modular Ambiguity Checking}

Ambiguity Requirement. In Figure 7 the two intersect cases are ambiguous, but neither CircleMod nor RectMod statically depends upon the other, so the ambiguity is not modularly detected. We address this problem by restricting EML's function extensibility such that cases declared in modules that do not statically depend upon one another are guaranteed to be unambiguous. Our restriction generalizes the implicit restrictions in standard functional and $\mathrm{OO}$ languages. First we introduce the concept of a function case's owner, which is the class (if any) at the owner position of the case's pattern. For example, ListSet is the owner of the second union case in Figure 2 because it appears at the owner position, while the first union case has no owner.

In functional languages, each case must be declared in the module that declares the associated function. In OO languages, each method must be declared inside the method's receiver. Our requirement is the disjunction of these conditions: every function case must either 1) be declared in the module that declares the case's function or 2) have an owner and be declared in the module that declares that owner.

RectMod now fails to typecheck because its intersect case does not satisfy our requirement: neither intersect nor Shape, the case's owner, is declared in RectMod. (An isomorphic error would occur in CircleMod if the second argument position in the pair were designated the owner position.) Therefore, RectMod may not extend intersect in that way. Removing that intersect case resolves the ambiguity for a pair of a Circle and a Rect. As another example, the add cases in HashSetMod and SortedListSetMod of Figures 3 and 4 are never compared for ambiguity, because the two modules do not statically depend upon one another. However, each case satisfies our requirement by following the traditional $\mathrm{OO}$ idiom of implementing an overriding case for a newly declared subclass of add's owner. Therefore the two cases are guaranteed to be unambiguous.

Since our ambiguity requirement is the disjunction of the implicit requirements in standard functional and OO languages, our requirement does not restrict those programming styles and allows them to coexist. Therefore, we have achieved our design goal of allowing the functional and $\mathrm{OO}$ extensibility idioms in a single class hierarchy while preserving modular type safety. ${ }^{6}$ However, other useful kinds of extensibility are disallowed by the ambiguity requirement. For example, a client of both UnionMod and HashSetMod from Figures 2 and 3 may want to implement a more efficient version of union for HashSets. However, the new case would violate our ambiguity requirement, so HashSets

${ }^{6}$ In the presence of multiple implementation inheritance, other kinds of ambiguities that elude modular detection can arise, necessitating an extra requirement [Millstein and Chambers 2002]. However, multiple interface inheritance, as in Java and EmL, cannot cause such ambiguities. 
are forced to use the default union case (or HashSetMod must be modified in place to add the new case).

Pairwise Ambiguity Checking. The above ambiguity requirement guarantees that each case declared in a structure $S$ is not ambiguous with cases that are unavailable from $S$. To complete modular ambiguity checking, each case $c$ declared in $S$ is checked for ambiguity with each available case $c^{\prime}$ other than itself. Let the patterns of $c$ and $c^{\prime}$ be Pat and Pat', respectively. Cases $c$ and $c^{\prime}$ are checked for ambiguity as follows:

- If Pat and Pat' are congruent, meaning that they are identical when all identifier bindings are removed, then the cases are ambiguous.

- Else if the patterns are disjoint, meaning that no value can match both Pat and Pat $^{\prime}$, then the cases are unambiguous.

- Else there is some pattern Pat" that represents the intersection of Pat and Pat': all values matching both Pat and Pat' also match Pat". The cases are unambiguous only if there exists a case $c^{\prime \prime}$ whose pattern is congruent to Pat". We call case $c^{\prime \prime}$ the resolving case, because it resolves the ambiguity between $c$ and $c^{\prime}$.

A degenerate form of the third scenario above occurs when one of Pat and Pat $^{\prime}$ is strictly more specific than the other, so the resolving case is one of the original two. For example, consider checking size for ambiguity in SetMod of Figure 1. The two size cases in Figure 1 are neither congruent nor disjoint. The intersection pattern is congruent to the second case's pattern, and that case itself is the resolving case.

\subsection{Discussion}

EML supports the extensibility idioms of traditional functional and OO languages: both new subclasses and new operations can be added to existing classes without modifying existing code, resolving a longstanding tension between these two forms of extensibility. At the same time, EML retains completely modular typechecking by imposing the modularity requirements described above.

The requirements rely heavily on the notion of a function's owner position. Although the owner position of a function $f$ does not affect $f$ 's invocation semantics, it has a large impact on the ways in which clients can interact with and extend the function. Through the local-default requirement, the owner position determines the default cases that clients must implement. Through the ambiguity requirement, the owner position determines what cases clients are forbidden from implementing. Therefore, effective use of $f$ by clients requires some advance planning by the original implementer of $f$, who must choose an appropriate owner position based on the kinds of extensibility that are anticipated. This advance planning is analogous to the need to choose which argument of a method should be the receiver in traditional OO languages. That choice has the same effects as the choice of the owner position in EML and additionally determines the sole argument that may be dynamically dispatched upon. 


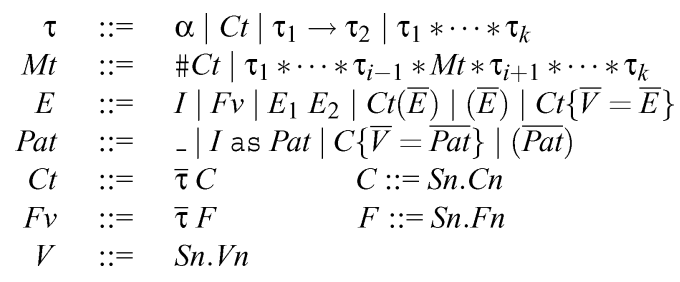

Fig. 8. Mini-EmL types, expressions, and patterns.

Intuitively, a class $C$ in $f$ 's argument type is a good choice as the function's owner if the following conditions hold:

- It is expected that $f$ 's behavior will depend on the particular subclass of $C$ that is passed as an argument.

- It is expected that clients will declare new subclasses of $C$.

The first condition implies that methods will likely need to dispatch at $C$ 's position. The second condition then implies that clients will likely need to declare overriding methods of $f$ for new subclasses of $C$. Making $C$ the owner allows these overriding methods to satisfy the ambiguity requirement. The first condition also implies that it may be difficult to implement $f$ for $C$ itself, if $C$ is abstract. Making $C$ the owner will obviate the need for such a default implementation, instead requiring concrete subclasses of $C$ to provide their own local defaults.

The EML exhaustiveness and ambiguity requirements together enforce an important monotonicity property on EML programs: the local view of a program from one structure is always consistent with the global view in a particular sense. Specifically, if the view from one structure suggests that some available type-correct argument $a$ to an available function $f$ will invoke the available case $c$, then that will be true at run time, no matter what other structures are part of the complete program. This property ensures that ITC can be safely performed piecewise on a function, from each structure's partial view of the program. It also validates a programmer's understanding given only a partial view of the program, ensuring that a type-correct argument cannot be "hijacked" by an unseen function case.

\section{MINI-EML}

This section describes Mini-EmL, a core language that formalizes EmL and its modular type system.

\subsection{Syntax}

Figure 8 defines the syntax of types, expressions, and patterns in Mini-EML. The syntax is essentially that of EML as informally presented so far, but some standard constructs are omitted, including base types, conditionals, anonymous and lexically nested functions, local variables, references, and exceptions. Metavariable $\alpha$ ranges over type variable names, $I$ over identifier names, $S n$ over structure names, $C n$ over class names, $V n$ over instance variable names, and $F n$ over 


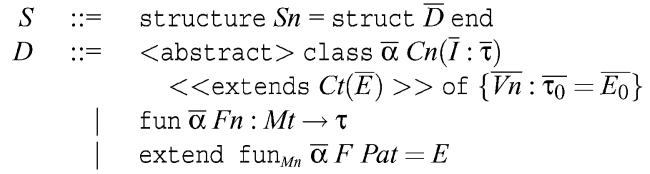

(a)

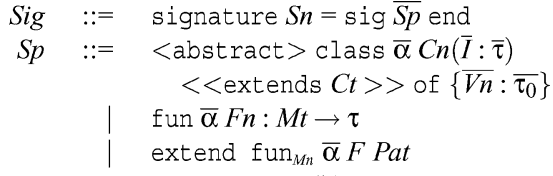

(b)

Fig. 9. (a) Mini-EmL structures and declarations. (b) Mini-EmL signatures and specifications.

function names. $\bar{X}$ denotes a comma-separated sequence of elements of the domain $X$ (and is independent of any variable named $X$ ); the empty sequence is denoted $\bullet$. The notation $\bar{V}=\bar{E}$ abbreviates $V_{1}=E_{1}, \ldots, V_{k}=E_{k}$ where $\bar{V}$ is $V_{1}, \ldots, V_{k}$ and $\bar{E}$ is $E_{1}, \ldots, E_{k}$ for some $k \geq 0$, and similarly for $\bar{V}=\overline{P a t}$.

MinI-EML types include type variables, class types, function types, and tuple types. The domain $M t$ represents marked types, which contain a \# mark on a single component class type. Expressions include identifiers, function values, function application, constructor calls, tuples, and instance expressions. The instance expression $C t\{\bar{V}=\bar{E}\}$ is not available at the source level, as instances may only be created via a constructor call $C t(\bar{E})$.

The construct $\{\bar{V}=\bar{E}\}$ differs from an ordinary record in two ways. First, the labels are scoped: the name of the structure in which an instance variable was introduced becomes part of the instance variable's name. Scoping allows a class to introduce an instance variable with the same name as one in a superclass declared in another module. While this ability provides only a minor convenience in Mini-EmL, scoping provides a foundation for allowing a class to safely hide instance variables, as described in Section 5. Instance variables in EmL use this mechanism implicitly; regular static scoping rules determine which instance variable is referred to. Second, for simplicity the components of $\{\bar{V}=\bar{E}\}$ are ordered, unlike traditional records.

Patterns include the wildcard pattern, identifier binding, class patterns, and tuple patterns. For simplicity, the representation pattern $\{\bar{V}=\overline{P a t}\}$ within a class pattern must mention all of the associated class's instance variables. A pattern of the form $I$, used in our earlier examples, is syntactic sugar for ( $I$ as _).

The notation and semantic style of Mini-EmL were influenced by Featherweight Java [Igarashi et al. 2001], a core language for Java. As in that language, classes are formally represented by their names. A class is uniquely represented as $S n$.Cn, where $C n$ is the name of the class and $S n$ is the name of the structure that declares $C n$. Functions are represented similarly.

The subset of expressions that are Mini-EmL values is described by the following grammar, which includes class instances, function values, and tuple values:

$$
v::=C t\{\bar{V}=\bar{v}\}|F v|(\bar{v})
$$

The syntax of structures and declarations is shown in Figure 9a. A structure consists of a sequence of class, extensible function, and function case declarations. The syntax of the three declarations is faithful to that of EML, except that cases now contain a case name, ranged over by metavariable $M n$. This name is used in the semantics to uniquely identify each function case 
declaration. Angle brackets $(<>)$ and double angle brackets $(<<>>)$ denote independent optional pieces of syntax. The notation $\overline{V n}: \bar{\tau}=\bar{E}$ abbreviates $V n_{1}: \tau_{1}=E_{1}, \ldots, V n_{k}: \tau_{k}=E_{k}$, and similarly for $\bar{I}: \bar{\tau}$. The declared inheritance graph is assumed to be acyclic. The class, function, and case names introduced in a given structure are assumed to be distinct. The type variables parameterizing a given declaration are assumed to be distinct. All the instance variable names introduced in a given structure are assumed to be distinct. The identifiers introduced in a given function case's pattern are assumed to be distinct.

Mini-EmL also includes an explicit notion of signature, as defined in Figure $9 \mathrm{~b}$. In ML, the name of a signature is completely independent of the names of structures that conform to the signature. However, there is a one-toone mapping between structures and signatures in a MinI-EML program (see below), so for simplicity the name of a MINI-EML structure is also used as the name of its associated signature. A signature contains a sequence of class, function, and function-case specifications. The syntax for each specification is identical to its corresponding declaration, but with all expressions removed. The notation $\overline{V n}: \bar{\tau}$ abbreviates $V n_{1}: \tau_{1}, \ldots, V n_{k}: \tau_{k}$.

Inspired by Featherweight Java, a MINI-EML program is a pair of a structure table and an expression to be evaluated. A structure table is a finite function from structure names to the associated structure declarations. The formal semantics assumes a fixed structure table $S T$. The domain of $S T$ is denoted $\operatorname{dom}(S T)$. The structure table is assumed to satisfy some sanity conditions: (1) $S T(S n)=$ (structure $S n=$ struct $\cdots$ end) for every $S n \in \operatorname{dom}(S T)$; (2) for every structure name $S n$ appearing anywhere in the program, $S n \in \operatorname{dom}(S T)$.

The static semantics also relies on a fixed signature table SigT, which maps each structure name $S n$ in $\operatorname{dom}(S T)$ to the principal signature of $S T(S n)$. SigT is easily computed from $S T$ : the principal signature of a structure $S$ is simply the signature of the same name as $S$ whose body is identical to $S$ 's body, but with all expressions removed. Each structure in the range of $S T$ is typechecked in the context of SigT, without access to $S T$, thereby ensuring that the first criterion for modular typechecking is met, as defined in Section 3.1: each structure is typechecked only against the signatures, rather than the implementations, of other structures. The domain of $\operatorname{Sig} T$ is denoted $\operatorname{dom}(\operatorname{Sig} T)$.

\subsection{Dynamic Semantics}

Mini-EmL's dynamic semantics is defined as a mostly standard small-step operational semantics. The structure table is accessed whenever information about a declaration is required in order to execute an expression. The metavariable $\rho$ ranges over environments, which are finite functions from identifiers to values. $|\bar{X}|$ denotes the length of the sequence $\bar{X}$. The notation $\left[I_{1} \mapsto\right.$ $\left.E_{1}, \ldots, I_{k} \mapsto E_{k}\right] X$ denotes the expression resulting from the simultaneous substitution of $E_{i}$ for each occurrence of $I_{i}$ in $X$, for $1 \leq i \leq k$, and similarly for $\left[\alpha_{1} \mapsto \tau_{1}, \ldots, \alpha_{k} \mapsto \tau_{k}\right] X .[\bar{I} \mapsto \bar{E}] X$ is used as a shorthand when $\bar{I}$ and $\bar{E}$ have the same length, and similarly for $[\bar{\alpha} \mapsto \bar{\tau}] X$. In a given inference rule, fragments enclosed in $<>$ must either be all present or all absent, and similarly 
$E \longrightarrow E^{\prime}$

$$
\begin{aligned}
& \frac{C t=(\bar{\tau} C) \quad \text { concrete }(C) \quad \operatorname{rep}\left(C t\left(\overline{E_{0}}\right)\right)=\left\{\bar{V}=\overline{E_{1}}\right\}}{C t\left(\overline{E_{0}}\right) \longrightarrow C t\left\{\bar{V}=\overline{E_{1}}\right\}} \text { E-NEW } \\
& \overline{C t}\left\{\overline{V_{0}}=\overline{v_{0}}, V=E, \overline{V_{1}}=\overline{E_{1}}\right\} \longrightarrow C t\left\{\overline{V_{0}}=\overline{v_{0}}, V=E^{\prime}, \overline{V_{1}}=\overline{E_{1}}\right\} \text { E-REP } \\
& \frac{E \longrightarrow E^{\prime}}{\left(\overline{v_{0}}, E, \overline{E_{1}}\right) \longrightarrow\left(\overline{v_{0}}, E^{\prime}, \overline{E_{1}}\right)} \text { E-TuP } \\
& \frac{E_{1} \longrightarrow E_{1}^{\prime}}{E_{1} E_{2} \longrightarrow E_{1}^{\prime} E_{2}} \text { E-APP1 } \frac{E_{2} \longrightarrow E_{2}^{\prime}}{v_{1} E_{2} \longrightarrow v_{1} E_{2}^{\prime}} \text { E-APP2 } \\
& \frac{\text { most-specifi c-case-for }(F v, v)=(\{(\bar{I}, \bar{v})\}, E)}{F v v \longrightarrow[\bar{I} \mapsto \bar{v}] E} \text { E-APPRED }
\end{aligned}
$$

concrete $(C)$

$$
\frac{(\operatorname{class} \bar{\alpha} C n \ldots) \in S T(S n)}{\operatorname{concrete}(S n . C n)} \text { CONCRETE }
$$

$\operatorname{rep}\left(C t\left(\overline{E_{0}}\right)\right)=\{\bar{V}=\bar{E}\}$

$\left(<<\right.$ abstract $>>$ class $\bar{\alpha} C n\left(\bar{I}: \overline{\tau_{1}}\right)<$ extends $C t\left(\overline{E_{0}}\right)>$ of $\left.\left\{\overline{V n}: \overline{\tau_{2}}=\overline{E_{2}}\right\}\right) \in S T(S n)$ $<\operatorname{rep}\left(C t\left(\overline{E_{0}}\right)\right)=\left\{\bar{V}=\overline{E_{1}}\right\}>$

$\operatorname{rep}((\bar{\tau} S n . C n)(\bar{E}))=[\bar{I} \mapsto \bar{E}][\bar{\alpha} \mapsto \bar{\tau}]\left\{<\bar{V}=\overline{E_{1}},>S n . \overline{V n}=\overline{E_{2}}\right\}$

most-specifi c-case-for $(F v, v)=(\rho, E)$

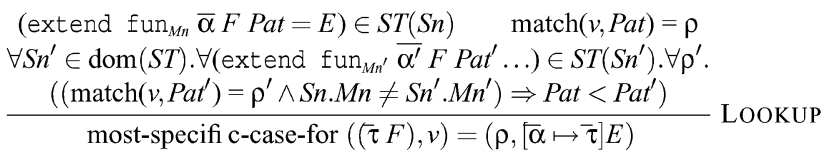

Fig. 10. Evaluation rules for MiNi-EmL expressions.

for $\langle<>>$. Sequences are sometimes treated as if they were sets. For example, $D \in \bar{D}$ means that $D$ is one of the declarations in $\bar{D}$. Finally, $D \in S T(S n)$ is shorthand for the two facts $S T(S n)=$ (structure $S n=$ struct $\bar{D}$ end) and $D \in \bar{D}$, and similarly for $S p \in \operatorname{Sig} T(S n)$.

Figure 10 contains the rules for evaluating expressions. The notation $(\bar{I}, \bar{v})$ abbreviates $\left(I_{1}, v_{1}\right), \ldots,\left(I_{k}, v_{k}\right)$, and $S n \cdot \overline{V n}=\bar{E}$ abbreviates $S n \cdot V n_{1}=$ $E_{1}, \ldots, S n . V n_{k}=E_{k}$. For simplicity in the semantics, a constructor call is treated as syntactic sugar for the instance expression obtained by expanding the constructor's definition. Rule E-New specifies this semantics, and Rule EREP evaluates instance expressions. It would be straightforward to instead use a call-by-value semantics for constructor calls, at the cost of some additional mechanism. Rule E-NEw uses the first two auxiliary rules at the bottom of the figure. Rule CONCRETE checks that the class to be instantiated was declared without the abstract keyword. Rule REP initializes the fields of the new instance as directed by the class's constructor, substituting the actual arguments to the 
constructor call for the formals. The rule also substitutes the new instance's type parameters for the class's type variables. Types have no dynamic effect in Mini-EmL, but maintaining types in the dynamic semantics eases the type system's proof of soundness.

The last rule in Figure 10 formalizes function-case lookup, used in E-APPRED. The first premise of Lookup specifies the case to invoke. The second premise ensures that this case is applicable: the argument value matches the case's pattern. That premise also produces an environment mapping each identifier in the case's pattern to the appropriate "pieces" of the argument value. This environment is used by E-APPRED to evaluate the case's body. The remaining premise ensures that the chosen case is most specific: the case is strictly more specific than any other applicable case. The condition $S n \cdot M n \neq S n^{\prime} . M n^{\prime}$ uses the case names to ensure that the chosen case is not compared for specificity with itself.

The rules for pattern matching and specificity are shown in Figure 11. The notation $\operatorname{match}(\bar{v}, \overline{P a t})=\bar{\rho}$ abbreviates $\operatorname{match}\left(v_{1}, P a t_{1}\right)=\rho_{1} \cdots \operatorname{match}\left(v_{k}\right.$, Pat $\left._{k}\right)=$ $\rho_{k}$, and similarly for $\overline{\text {Pat }_{1}} \leq \overline{\text { Pat }_{2}}$. The matching rules are straightforward except for E-MatchClass. The judgment $C \leq C^{\prime}$ is defined at the bottom of Figure 11 as the reflexive, transitive closure of the declared class extends relation. Therefore, an instance of class $C$ matches a class pattern of class $C^{\prime}$ if $C$ subclasses $C^{\prime}$ and the instance's representation recursively matches the given representation pattern. The instance may have more instance variables than are mentioned in the given representation pattern, so that subclass instances can match superclass patterns.

The judgment Pat $\leq P a t^{\prime}$ means that Pat is at least as specific as Pat'. Rule LookuP uses Pat $<$ Pat' as shorthand for the two facts Pat $\leq$ Pat $t^{\prime}$ and Pat $\not$ Pat. The pattern specificity semantics generalizes OO-style best-match semantics to support ML-style patterns. Any pattern is at least as specific as the wildcard, and identifier binding has no effect on specificity. Class pattern specificity (SPECClAsS) follows the ordering induced by subclassing. Analogous with E-MATCHCLASS, the more-specific pattern may contain extra instance variables. The natural rule SPECTup for specificity of tuple patterns is analogous to the symmetric dispatching semantics of multimethod-based OO languages like Dubious [Millstein and Chambers 2002]. When a tuple is used to send multiple arguments to a function, tuple patterns allow all arguments to be dynamically dispatched upon, and no argument position is more important than the rest.

\subsection{Static Semantics}

Figure 12 contains the rules for typechecking structures and declarations. $\Gamma$ is a type environment, mapping identifiers to types. The notation $\hat{M} t$ denotes the type $\tau$ identical to $M t$, but with the \# mark removed. The notation $\overline{S n} \vdash$ $\bar{D}$ OK in $S n$ abbreviates $\overline{S n} \vdash D_{1}$ OK in $S n \ldots \overline{S n} \vdash D_{k}$ OK in $S n ; \bar{\alpha} \vdash \bar{\tau}$ OK abbreviates $\bar{\alpha} \vdash \tau_{1}$ OK $\cdots \bar{\alpha} \vdash \tau_{k}$ OK; $(\bar{I}, \bar{\tau})$ abbreviates $\left(I_{1}, \tau_{1}\right), \ldots,\left(I_{k}, \tau_{k}\right) ; \Gamma ; \bar{\alpha} \vdash$ $\bar{E}: \bar{\tau}$ abbreviates $\Gamma ; \bar{\alpha} \vdash E_{1}: \tau_{1} \cdots \Gamma ; \bar{\alpha} \vdash E_{k}: \tau_{k} ; \bar{\tau}_{1} \leq \overline{\tau_{0}}$ abbreviates $\tau_{11} \leq$ $\tau_{01} \cdots \tau_{1 k} \leq \tau_{0 k}$. 
$\operatorname{match}(v$, Pat $)=\rho$

$$
\begin{aligned}
& \overline{\operatorname{match}\left(v,_{-}\right)=\{\}} \text {E-MATCHWILD } \\
& \frac{\operatorname{match}(v, \text { Pat })=\rho}{\operatorname{match}(v, I \text { as } \text { Pat })=\rho \cup\{(I, v)\}} \text { E-MATCHBIND } \\
& \frac{C \leq C^{\prime} \quad \operatorname{match}(\bar{v}, \overline{P a t})=\bar{\rho}}{\operatorname{match}\left(\bar{\tau} C\left\{\bar{V}=\bar{v}, \overline{V_{1}}=\overline{v_{1}}\right\}, C^{\prime}\{\bar{V}=\overline{P a t}\}\right)=\bigcup \bar{\rho}} \text { E-MATCHCLASS } \\
& \frac{\operatorname{match}(\bar{v}, \overline{P a t})=\bar{\rho}}{\operatorname{match}((\bar{v}),(\overline{P a t}))=\bigcup \bar{\rho}} \text { E-MATCHTUP }
\end{aligned}
$$

Pat $\leq$ Pat'

$$
\begin{aligned}
& \overline{\text { Pat } \leq \text { - SPECWILD }} \\
& \frac{\text { Pat }_{1} \leq \text { Pat }_{2}}{I \text { as } \text { Pat }_{1} \leq \text { Pat }_{2}} \text { SPECBIND } 1 \quad \frac{\text { Pat }_{1} \leq \text { Pat }_{2}}{\text { Pat }_{1} \leq I \text { as Pat }} \text { SPECBIND2 } \\
& \frac{C \leq C^{\prime} \quad \overline{\text { Pat }_{1}} \leq \overline{\text { Pat }_{2}}}{C\left\{\bar{V}=\overline{\text { Pat }_{1}}, \overline{V_{3}}=\overline{\text { Pat }_{3}}\right\} \leq C^{\prime}\left\{\bar{V}=\overline{\text { Pat }_{2}}\right\}} \text { SPECCLASS } \\
& \frac{\overline{\text { Pat }_{1}} \leq \overline{\text { Pat }_{2}}}{\left(\overline{\text { Pat }_{1}}\right) \leq\left(\overline{\text { Pat }_{2}}\right)} \text { SPECTUP }
\end{aligned}
$$

$C \leq C^{\prime}$

$$
\begin{gathered}
\overline{C \leq C} \text { SubReF } \\
\frac{C_{1} \leq C_{2} \quad C_{2} \leq C_{3}}{C_{1} \leq C_{3}} \text { SuBTRAnS } \\
\frac{\left(<\text { abstract }>\text { class } \bar{\alpha} C n\left(\overline{I_{1}}: \overline{\tau_{1}}\right) \text { extends } \bar{\tau} C \ldots\right) \in S T(S n)}{S n . C n \leq C} \text { SuBEXT }
\end{gathered}
$$

Fig. 11. EmL pattern matching, pattern specificity, and subclassing.

Structures are typechecked (STRUCTOK) by checking each declaration in turn. It is assumed that $S$ OK holds for each structure $S$ in the range of $S T . \overline{S n}$ in STRUCtOK denotes those signatures in SigT that may be accessed during ITC on the current structure. As defined in Section 3.1, ITC is only modular if the current structure statically depends upon each signature in $\overline{S n}$. Later judgments ensure the well-formedness of the chosen $\overline{S n}$, as described below. ${ }^{7}$

\footnotetext{
${ }^{7}$ This "guess and check" style is used for simplicity. An alternative would be to take an initial pass over each structure's declarations in the static semantics, in order to compute the appropriate $\overline{S n}$ before typechecking the structure.
}

ACM Transactions on Programming Languages and Systems, Vol. 26, No. 5, September 2004. 
$S$ OK

$$
\frac{\overline{S n} \subseteq \operatorname{dom}(\operatorname{Sig} T) \quad \overline{S n} \vdash \bar{D} \text { OK in } S n}{\text { structure } S n=\operatorname{struct} \bar{D} \text { end OK }} \text { STRUCTOK }
$$

$\overline{S n} \vdash D$ OK in $S n$

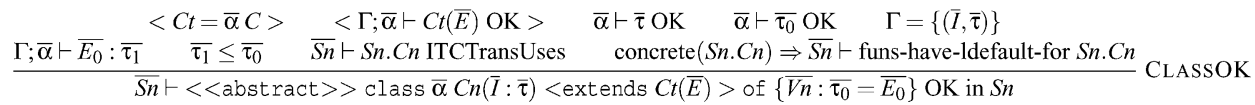

$$
\begin{aligned}
& \begin{array}{lll}
\bar{\alpha} \vdash \hat{M} t \text { OK } & \bar{\alpha} \vdash \tau \text { OK } & \text { owner }(S n . F n)=S n^{\prime} . C n \quad S n \neq S n^{\prime} \Rightarrow \overline{S n} \vdash S n . F n \text { has-gdefault } \\
\hline \overline{S n} \vdash \text { fun } \bar{\alpha} F n: M t \rightarrow \tau \text { OK in } S n
\end{array} \\
& \left(\text { fun } \overline{\alpha^{\prime}} F n: M t \rightarrow \tau\right) \in \operatorname{Sig} T\left(S n^{\prime}\right) \quad \text { matchType }\left(\left[\overline{\alpha^{\prime}} \mapsto \bar{\alpha}\right] \hat{M} t, P a t\right)=\left(\Gamma, \tau_{0}\right) \\
& \frac{\Gamma ; \bar{\alpha} \vdash E: \tau^{\prime} \quad \tau^{\prime} \leq\left[\overline{\alpha^{\prime}} \mapsto \bar{\alpha}\right] \tau \quad \overline{S n} \vdash S n^{\prime} . F n \text { ITCUses } \quad S n ; \overline{S n} \vdash \text { extend fun }{ }_{M n} \bar{\alpha} S n^{\prime} . F n \text { Pat unambiguous }}{\overline{S n} \vdash \text { extend } \text { fun }_{M n} \bar{\alpha} S n^{\prime} . F n \text { Pat }=E \text { OK in } S n} \text { CASEOK }
\end{aligned}
$$

Fig. 12. Static semantics of Mini-EmL structures and declarations.

The formalism does not explicitly enforce modularity of the rest of static typechecking, as those checks are standard and are naturally modular.

The rules for typechecking the three declaration forms are largely straightforward. Rule CLASSOK checks that a class's superclass constructor call is welltyped, that all types mentioned in the class declaration are well-formed, and that the instance-variable initializer expressions have the appropriate types. The first premise in the rule ensures that the new class has the same type variables as its superclass, as mentioned in Section 2.5. Rule FunOK checks that a function's declared type is well-formed. Rule CASEOK ensures that the case's pattern and body are compatible with the associated function's declared type. The "ITCTransUses" and "ITCUses" judgments in CLASsOK and FunOK ensure well-formedness of the signatures $\overline{S n}$ to be accessed during ITC of the enclosing structure; these judgments are described below. Finally, each rule enforces one of the three requirements for modular ITC: CLASsOK enforces the localdefault requirement ("funs-have-ldefault-for") if the class is concrete; FunOK enforces the global-default requirement ("has-gdefault") if the function is external; CASEOK performs ambiguity checking ("unambiguous") for the given case, which includes enforcement of the ambiguity requirement as described in Section 3 . The judgment for each requirement has $\overline{S n}$ in the context, to ensure that only signatures in this sequence are accessed during enforcement of the requirement.

Figure 13 contains the static semantics of types. The judgment $\bar{\alpha} \vdash \tau$ OK ensures that $\tau$ refers only to type variables in $\bar{\alpha}$ and that each class in $\tau$ has the correct number of type parameters. The subtyping relation $\tau \leq \tau^{\prime}$ is completely standard [Cardelli 1988].

Figure 14 contains the rules for typechecking expressions. The notation $S n . \overline{V n}: \bar{\tau}$ abbreviates $S n . V n_{1}: \tau_{1}, \ldots, S n . V n_{k}: \tau_{k}$. The judgment $\Gamma ; \bar{\alpha} \vdash E: \tau$ ensures that an expression is well-typed in the context of the type environment and sequence of type variables currently in scope. Most of the rules are standard. Rule T-Fun looks up a function's declared type in the signature table and substitutes the given use's type parameters for the function's type variables. Rule T-New uses T-ConsTR to check that a constructor invocation includes a 
$\bar{\alpha} \vdash \tau \mathrm{OK}$

$$
\begin{aligned}
& \frac{\alpha \in \bar{\alpha}}{\bar{\alpha} \vdash \alpha \text { OK }} \text { TVAROK } \\
& \bar{\alpha} \vdash \bar{\tau} \text { OK } \\
& \left(<\text { abstract }>\text { class } \overline{\alpha_{0}} \mathrm{Cn} \ldots\right) \in \operatorname{Sig} T(S n) \\
& \left|\overline{\alpha_{0}}\right|=|\bar{\tau}| \\
& \bar{\alpha} \vdash \bar{\tau} S n . C n \text { OK } \\
& \frac{\bar{\alpha} \vdash \tau_{1} \text { OK } \quad \bar{\alpha} \vdash \tau_{2} \text { OK }}{\bar{\alpha} \vdash \tau_{1} \rightarrow \tau_{2} \text { OK }} \text { FUNTYPEOK } \\
& \frac{\bar{\alpha} \vdash \tau_{1} \text { OK } \cdots \bar{\alpha} \vdash \tau_{k} \text { OK }}{\bar{\alpha} \vdash \tau_{1} * \cdots * \tau_{k} \text { OK }} \text { TUPTYPEOK }
\end{aligned}
$$

$\tau \leq \tau^{\prime}$

$$
\begin{aligned}
& \overline{\tau \leq \tau} \text { SUBTReF } \frac{\tau_{1} \leq \tau_{2} \quad \tau_{2} \leq \tau_{3}}{\tau_{1} \leq \tau_{3}} \text { SUbTTRANS } \\
& \text { (<abstract > class } \bar{\alpha} C n\left(\overline{I_{1}}: \overline{\tau_{1}}\right) \\
& \frac{\text { extends } C t \ldots) \in \operatorname{Sig} T(S n)}{\bar{\tau} \operatorname{Sn} . C n \leq[\bar{\alpha} \mapsto \bar{\tau}] C t} \text { SUBTEXT } \\
& \frac{\tau_{1}^{\prime} \leq \tau_{1} \quad \tau_{2} \leq \tau_{2}^{\prime}}{\tau_{1} \rightarrow \tau_{2} \leq \tau_{1}^{\prime} \rightarrow \tau_{2}^{\prime}} \text { SUBTFUN } \\
& \frac{\tau_{1} \leq \tau_{1}^{\prime} \cdots \tau_{k} \leq \tau_{k}^{\prime}}{\tau_{1} * \cdots * \tau_{k} \leq \tau_{1}^{\prime} * \cdots * \tau_{k}^{\prime}} \text { SUBTTUP }
\end{aligned}
$$

Fig. 13. Static semantics of MinI-EmL types.

$\Gamma ; \bar{\alpha} \vdash E: \tau$

$$
\begin{aligned}
& \frac{(I, \tau) \in \Gamma}{\Gamma ; \bar{\alpha} \vdash I: \tau} \text { T-ID } \\
& \frac{\left(\text { fun } \overline{\alpha_{0}} F n: M t \rightarrow \tau\right) \in \operatorname{Sig} T(S n) \quad \bar{\alpha} \vdash \overline{\tau_{0}} \text { OK }}{\Gamma ; \bar{\alpha} \vdash \overline{\tau_{0}} \operatorname{Sn} . F n:\left[\overline{\alpha_{0}} \mapsto \overline{\tau_{0}}\right](\hat{M} t \rightarrow \tau)} \text { T-FUN } \\
& \frac{\Gamma ; \bar{\alpha} \vdash E_{1}: \tau_{2} \rightarrow \tau \quad \Gamma ; \bar{\alpha} \vdash E_{2}: \tau_{2}^{\prime} \quad \tau_{2}^{\prime} \leq \tau_{2}}{\Gamma ; \bar{\alpha} \vdash E_{1} E_{2}: \tau} \text { T-APP } \\
& \frac{\Gamma ; \bar{\alpha} \vdash C t(\bar{E}) \text { OK } \quad C t=(\bar{\tau} C) \quad \text { concrete }(C)}{\Gamma ; \bar{\alpha} \vdash C t(\bar{E}): C t} \text { T-NEW } \\
& \frac{\Gamma ; \bar{\alpha} \vdash E_{1}: \tau_{1} \cdots \Gamma ; \bar{\alpha} \vdash E_{k}: \tau_{k}}{\Gamma ; \bar{\alpha} \vdash\left(E_{1}, \ldots, E_{k}\right): \tau_{1} * \cdots * \tau_{k}} \text { T-TUP } \\
& \begin{array}{ccccc}
\bar{\alpha} \vdash C t \text { OK } & C t=\left(\overline{\tau_{0}} C\right) \quad \text { concrete }(C) \quad \text { repType }(C t)=\{\bar{V}: \bar{\tau}\} & \Gamma ; \bar{\alpha} \vdash \bar{E}: \overline{\tau_{1}} & \overline{\tau_{1}} \leq \bar{\tau} \\
\Gamma ; \bar{\alpha} \vdash C t\{\bar{V}=\bar{E}\}: C t & \text { T-REP }
\end{array}
\end{aligned}
$$

$\Gamma ; \bar{\alpha} \vdash C t(\bar{E})$ OK

$$
\begin{gathered}
\bar{\alpha} \vdash C t \text { OK } \\
C t=\left(\overline{\tau_{0}} S n . C n\right) \quad\left(<\text { abstract }>\text { class } \overline{\alpha_{0}} C n(\bar{I}: \bar{\tau}) \ldots\right) \in S i g T(S n) \quad \Gamma ; \bar{\alpha} \vdash \bar{E}: \overline{\tau_{1}} \quad \overline{\tau_{1}} \leq\left[\overline{\alpha_{0}} \mapsto \overline{\tau_{0}}\right] \bar{\tau} \\
\Gamma ; \bar{\alpha} \vdash C t(\bar{E}) \text { OK }
\end{gathered}
$$

$\operatorname{repType}(C t)=\{\bar{V}: \bar{\tau}\}$

$$
\begin{gathered}
\begin{array}{c}
\left(<<\text { abstract }>>\text { class } \bar{\alpha} C n\left(\bar{I}: \overline{\tau_{1}}\right)<\text { extends } C t>\text { of }\left\{\overline{V n}: \overline{\tau_{2}}\right\}\right) \in \operatorname{Sig} T(S n) \\
<\operatorname{repType}(C t)=\left\{\bar{V}: \overline{\tau_{3}}\right\}>
\end{array} \\
\operatorname{repType}(\bar{\tau} S n . C n)=[\bar{\alpha} \mapsto \bar{\tau}]\left\{\left\langle\bar{V}: \overline{\tau_{3}},>\operatorname{Sn} \cdot \overline{V n}: \overline{\tau_{2}}\right\}\right. \\
\text { REPTYPE }
\end{gathered}
$$

Fig. 14. Static semantics of Mini-EmL expressions.

well-formed class type and that the actual arguments have appropriate types, as dictated by the class's specification. Rule T-REP ensures that an instance expression includes a well-formed class type and that the instance-variable expressions have appropriate types, as dictated by the class's specification. Rule T-REP uses REPTYPE, which computes a class's representation type.

Figure 15 contains the rules for typechecking patterns. The notation $\operatorname{matchType}(\bar{\tau}, \overline{P a t})=\left(\bar{\Gamma}, \overline{\tau^{\prime}}\right)$ abbreviates matchType $\left(\tau_{1}, P_{1} t_{1}\right)=\left(\Gamma_{1}, \tau_{1}^{\prime}\right) \ldots$ 
$\operatorname{matchType}(\tau, P a t)=\left(\Gamma, \tau^{\prime}\right)$

$$
\begin{aligned}
& \overline{\operatorname{matchType}\left(\tau,{ }_{-}\right)=(\{\}, \tau)} \text { T-MATCHWILD } \\
& \frac{\operatorname{matchType}(\tau, \text { Pat })=\left(\Gamma, \tau^{\prime}\right)}{\operatorname{matchType}(\tau, I \text { as } \text { Pat })=\left(\Gamma \cup\left\{\left(I, \tau^{\prime}\right)\right\}, \tau^{\prime}\right)} \text { T-MATChBIND } \\
& \frac{C \leq C^{\prime} \quad \operatorname{repType}(\bar{\tau} C)=\left\{\bar{V}: \overline{\tau_{0}}\right\} \quad \text { matchType }\left(\overline{\tau_{0}}, \overline{P a t}\right)=\left(\bar{\Gamma}, \overline{\tau_{1}}\right)}{\text { matchType }\left(\left(\bar{\tau} C^{\prime}\right), C\{\bar{V}=\overline{P a t}\}\right)=(\bigcup \bar{\Gamma},(\bar{\tau} C))} \text { T-MaTCHCLASS } \\
& \frac{\operatorname{matchType}\left(\tau_{1}, \text { Pat }_{1}\right)=\left(\Gamma_{1}, \tau_{1}^{\prime}\right) \cdots \text { matchType }\left(\tau_{k}, \text { Pat }_{k}\right)=\left(\Gamma_{k}, \tau_{k}^{\prime}\right)}{\operatorname{matchType}\left(\tau_{1} * \cdots * \tau_{k},\left(\text { Pat }_{1}, \ldots, \text { Pat }_{k}\right)\right)=\left(\Gamma_{1} \cup \ldots \cup \Gamma_{k}, \tau_{1}^{\prime} * \cdots * \tau_{k}^{\prime}\right)} \text { T-MATCHTUP }
\end{aligned}
$$

Fig. 15. Static semantics of Mini-EmL patterns.

$\overline{S n} \vdash$ Sn.Cn ITCTransUses

$$
\begin{gathered}
\begin{array}{c}
\left(<<\text { abstract }>>\text { class } \bar{\alpha} \operatorname{Cn}(\bar{I}: \bar{\tau})<\text { extends }\left(\overline{\tau_{0}} C\right)>\ldots\right) \in \operatorname{SigT}(S n) \\
S n \in \overline{S n} \quad<\overline{S n} \vdash C \text { ITCTransUses }>
\end{array} \\
\overline{S n} \vdash \text { Sn.Cn ITCTransUses }
\end{gathered}
$$

$\overline{S n} \vdash F$ ITCUses

$$
\frac{S n \in \overline{S n}}{\overline{S n} \vdash S n \cdot F n \text { ITCUses }} \text { FunITCUSES }
$$

Fig. 16. Well-formedness of the signatures to be accessed during ITC of a structure.

matchType $\left(\tau_{k}, P a t_{k}\right)=\left(\Gamma_{k}, \tau_{k}^{\prime}\right)$. The judgment matchType $(\tau, P a t)=\left(\Gamma, \tau^{\prime}\right)$ checks that a pattern is compatible with type $\tau$. The judgment produces a type environment mapping the identifiers bound in Pat to their types. This type environment is used in CASEOK (Figure 12) to typecheck the associated case's body. The type $\tau^{\prime}$ represents the particular subtype of $\tau$ to which Pat conforms; it is used to give precise types to any identifiers bound to Pat, as shown in rule T-MATCHBind.

Figure 16 contains the well-formedness rules for the signatures $\overline{S n}$ (which are "guessed" in STRUCTOK) to be accessed during ITC of a structure $\mathrm{Sn}$. Rule ClassITCTRansUses is used by ClassOK in Figure 12 to ensure that $\overline{S n}$ contains all the signatures that specify a (reflexive, transitive) superclass of a class declared in $S n$. Rule FunITCUSES is used by CASEOK to ensure that $\overline{S n}$ contains the signature specifying the associated function for a case declared in $S n$. In either case, if $\overline{S n}$ is required to include some signature $S n^{\prime}$, then $S n$ does indeed statically depend upon $S n^{\prime}$ according to the definition of static dependency given in Section 3.1. The rules do not ensure that all statically depended upon signatures are in $\overline{S n}$, but only those required for precise modular ITC. The rules also do not explicitly forbid $\overline{S n}$ from including signatures that are not statically depended upon. However, the type soundness proof for MinI-EML can only rely on the two properties of $\overline{S n}$ enforced by rules ClassITCTRANsUses and FunITCUses. Therefore, the proof validates the safety of modular ITC. 
$\overline{S n} \vdash F$ has-gdefault

$$
\frac{\text { owner }(F)=C \quad \overline{S n} \vdash F \text { has-default-for } C}{\overline{S n} \vdash F \text { has-gdefault }} \text { GDEFAULT }
$$

$\overline{S n} \vdash$ funs-have-ldefault-for $C$

$$
\frac{\forall F, C^{\prime} .\left[\left(\overline{S n} \vdash F \text { ITCUses } \wedge \text { owner }(F)=C^{\prime} \wedge C \leq C^{\prime}\right) \Rightarrow \overline{S n} \vdash F \text { has-default-for } C\right]}{\overline{S n} \vdash \text { funs-have-ldefault-for } C}
$$

$\overline{S n} \vdash F$ has-default-for $C$

$$
\begin{aligned}
& (\text { fun } \bar{\alpha} F n: M t \rightarrow \tau) \in \operatorname{Sig} T(S n)
\end{aligned}
$$

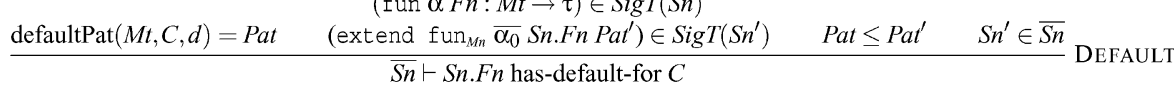

$\operatorname{defaultPat}(T, C, d)=P a t$

$$
\begin{aligned}
& \overline{\text { defaultPat }(T, C, 0){ }_{-}} \text {DEFZERO } \\
& \frac{d>0}{\operatorname{defaultPat}(\alpha, C, d)=_{-}} \text {DEFTYPEVAR } \\
& \frac{\operatorname{repType}\left(\bar{\tau} C^{\prime}\right)=\left\{\bar{V}: \overline{\tau_{0}}\right\} \quad \operatorname{defaultPat}\left(\overline{\tau_{0}}, C, d-1\right)=\overline{\text { Pat }} \quad d>0}{\operatorname{defaultPat}\left(\left(\bar{\tau} C^{\prime}\right), C, d\right)=\left(C^{\prime}\{\bar{V}=\overline{\text { Pat }}\}\right)} \text { DefClassTyPE } \\
& \frac{\operatorname{repType}(\bar{\tau} C)=\left\{\bar{V}: \overline{\tau_{0}}\right\} \quad \operatorname{defaultPat}\left(\overline{\tau_{0}}, C, d-1\right)=\overline{\text { Pat }} \quad d>0}{\operatorname{defaultPat}\left(\#\left(\bar{\tau} C^{\prime}\right), C, d\right)=(C\{\bar{V}=\overline{\text { Pat }}\})} \text { DefOwNERCLASSTYPE } \\
& \frac{\operatorname{defaultPat}\left(T_{1}, C, d-1\right)=\text { Pat }_{1} \cdots \text { defaultPat }\left(T_{k}, C, d-1\right)=\text { Pat }_{k} \quad d>0}{\operatorname{defaultPat}\left(T_{1} * \ldots * T_{k}, C, d\right)=\left(\text { Pat }_{1}, \ldots, \text { Pat }_{k}\right)} \text { DefTupTYPE } \\
& \frac{d>0}{\operatorname{defaultPat}\left(\tau_{1} \rightarrow \tau_{2}, C, d\right)=_{-}} \text {DefFunTYPE }
\end{aligned}
$$

Fig. 17. Modular exhaustiveness checking for Mini-EmL.

Figure 17 formalizes the portion of modular ITC that ensures functions are exhaustive. The notation defaultPat $(\bar{\tau}, C, d)=\overline{P a t}$ abbreviates $\operatorname{defaultPat}\left(\tau_{1}, C, d\right)=\operatorname{Pat}_{1} \cdots$ defaultPat $\left(\tau_{k}, C, d\right)=\operatorname{Pat}_{k}$. Metavariable $T$ ranges over both types and marked types, and metavariable $d$ ranges over nonnegative integers. Rule GDEFAULT checks that a given function has a global default case, and LDEFAULT checks that all available functions whose owners are superclasses of a given class $C$ have a local default case for $C$. Since a global default case of $F$ is equivalent to a local default case of $F$ for $C$, where $C$ is the owner of $F$, the two requirements are able to share the helper rule Default that performs the checks.

The global- and local-default requirements are enforced by the algorithm described in Section 3 above. Rule DEFAULT generates a (global or local) default pattern and checks that this pattern is at least as specific as the pattern of some available function case. The judgment $\operatorname{defaultPat}(T, C, d)=P a t$ generates a default pattern of (possibly marked) type $T$. The default pattern dispatches on $C$ in the marked position (if any) of $T$ and accepts any type-correct argument in the other positions. The integer $d$ represents the depth that the generated 
$S n ; \overline{S n} \vdash$ extend fun $_{M n} \bar{\alpha} F$ Pat unambiguous

$$
\begin{aligned}
& \left(\text { fun } \overline{\alpha_{1}} F n: M t \rightarrow \tau\right) \in \operatorname{Sig} T\left(S n_{1}\right) \quad S n=S n_{1} \vee \text { owner }(M t, P a t)=S n . C n \\
& \forall S n^{\prime} \in \overline{S n} . \forall\left(\text { extend } \operatorname{fun}_{M n^{\prime}} \overline{\alpha_{1}} F P a t^{\prime}\right) \in \operatorname{Sig} T\left(S n^{\prime}\right) \text {. } \\
& \left(S n . M n \neq S n^{\prime} . M n^{\prime} \Rightarrow \overline{S n} \vdash\right. \text { (Pat,Pat') unambiguous) } \\
& S n ; \overline{S n} \vdash \text { extend } \text { fun }_{M n} \bar{\alpha} S n_{1} . F n \text { Pat unambiguous }
\end{aligned}
$$

$\overline{S n} \vdash($ Pat, Pat' $)$ unambiguous

$$
\begin{aligned}
& \text { Pat } \neq \text { Pat } \\
& \forall \text { Pat }_{0} \cdot\left(\left(\text { Pat }_{\text {Pat }}{ }^{\prime}=\text { Pat }_{0}\right) \Rightarrow\right. \\
& \frac{\left.\exists S n^{\prime \prime} \in \overline{S n} \cdot \exists\left(\text { extend } \text { fun }_{M n^{\prime \prime}} \overline{\alpha_{2}} F P a t^{\prime \prime}\right) \in \operatorname{Sig} T\left(S n^{\prime \prime}\right) \cdot\left(P a t_{0} \cong P a t^{\prime \prime}\right)\right)}{\overline{S n} \vdash\left(P a t, P a t^{\prime}\right) \text { unambiguous }} \text { PAIRAMB }
\end{aligned}
$$

$\mathrm{Pat}_{1} \cap \mathrm{Pat}_{2}=\mathrm{Pat}$

$$
\begin{aligned}
& \frac{{ }_{-} \cap \text { Pat }=\text { Pat }}{\text { PatInt Wild }} \quad \frac{\text { Pat }_{1} \cap \text { Pat }_{2}=\text { Pat }}{I \text { as } \text { Pat }_{1} \cap \text { Pat }_{2}=\text { Pat }} \text { PatIntBind }
\end{aligned}
$$

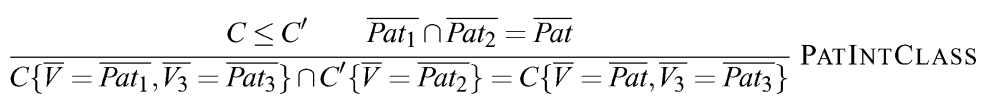

$$
\begin{aligned}
& \frac{\overline{\text { Pat }_{1}} \cap \overline{\text { Pat }_{2}}=\overline{\text { Pat }}}{\left(\overline{\text { Pat }_{1}}\right) \cap\left(\overline{\text { Pat }_{2}}\right)=(\overline{P a t})} \text { PATIntTup } \quad \frac{\text { Pat }_{2} \cap \text { Pat }_{1}=\text { Pat }}{\text { Pat }_{1} \cap P a t_{2}=\text { Pat }} \text { PATIntRev }
\end{aligned}
$$

Fig. 18. Modular ambiguity checking for Mini-EmL.

pattern should have. Rule DEFAULT chooses the depth non-deterministically, and the Mini-EmL type soundness proof implies that any depth can safely be used. However, as discussed in Section 3, an implementation of the algorithm should choose a large-enough depth to ensure precision.

Figure 18 formalizes the portion of modular ITC that ensures functions are unambiguous. The notation $\overline{P a t^{\prime}} \cap \overline{P a t^{\prime \prime}}=\overline{P a t}$ abbreviates $P a t_{1}^{\prime} \cap P a t_{1}^{\prime \prime}=P a t_{1} \ldots$ $\mathrm{Pat}_{k}^{\prime} \cap \mathrm{Pat}_{k}^{\prime \prime}=\mathrm{Pat}_{k}$. The top-level rule is Aмв. The second premise enforces the ambiguity requirement, ensuring that the given function case is declared in the same module as either its associated function or its owner. The final premise in AMB uses PAIRAmB to check that the given case is unambiguous with each available function case other than itself.

PAIRAMB uses the pairwise ambiguity algorithm described in Section 3 . $P a t \cong P a t^{\prime}$ denotes that Pat is congruent to Pat'; it is an abbreviation for the two facts Pat $\leq$ Pat $t^{\prime}$ and $P a t^{\prime} \leq$ Pat. Pattern intersection is formalized by the judgment $P a t \cap P a t^{\prime}=P^{\prime \prime} t^{\prime \prime}$. If Pat and Pat are disjoint then there is no Pat" such that $P a t \cap P a t^{\prime}=P a t^{\prime \prime}$. The rules for pattern intersection are straightforward. The intersection of class patterns (rule PATINTClass) is simplified by the fact that classes support only single inheritance. In particular, if two class patterns are applicable to a common value, then one class must be a subclass of the other.

Figure 19 contains the helper judgments for accessing the class at the owner position of a function, type, and pattern. Finally, the rules defining the 
owner $(F)=C$

$$
\frac{(\text { fun } \bar{\alpha} F n: M t \rightarrow \tau) \in \operatorname{Sig} T(S n) \quad \text { owner }(M t)=C}{\text { owner }(S n . F n)=C} \text { OWNERFun }
$$

$\operatorname{owner}(M t)=C$

$$
\begin{gathered}
\text { owner }(\# \bar{\tau} C)=C \\
\text { Owner }(M t)=C \\
\frac{\operatorname{owner}\left(\tau_{1} * \cdots * \tau_{i-1} * M t * \tau_{i+1} * \cdots * \tau_{k}\right)=C}{\text { OWNERTUP }}
\end{gathered}
$$

$\operatorname{owner}(M t$, Pat $)=C$

$$
\begin{gathered}
\frac{\text { owner }(M t, \text { Pat })=C}{\text { owner }(M t, I \text { as Pat })=C} \text { OwnerBindPat } \\
\frac{\text { owner }\left(M t, \text { Pat }_{i}\right)=C}{\text { owner }\left(\tau_{1} * \cdots * \tau_{i-1} * M t * \tau_{i+1} * \cdots * \tau_{k},\left(\text { Pat }_{1}, \ldots, \text { Pat }_{k}\right)\right)=C} \text { OWNERTuPPAT } \\
\frac{\text { owner }(\# C t, C\{\bar{V}=\overline{\text { Pat }}\})=C}{\text { OWNERCLASSPAT }}
\end{gathered}
$$

Fig. 19. Accessing the owner.

judgments concrete $(C), P a t \leq P a t^{\prime}$, and $C \leq C^{\prime}$ in Figures 10 and 11 are borrowed from the dynamic semantics. ${ }^{8}$

\subsection{Type Soundness}

Mini-EmL's type system is sound: a well-typed Mini-EmL program cannot incur type errors at run time. Mini-EmL's type errors are defined implicitly by the stuck expressions, which are those expressions that are not values but cannot be further evaluated (because there is no applicable rule in the Mini-EmL dynamic semantics). The match nonexhaustive and match ambiguous type errors are represented by the fact that function invocations lacking a most-specific applicable function case are stuck in the Mini-EmL dynamic semantics. Mini-EmL's soundness therefore validates the correctness of modular ITC, showing that it is sufficient to ensure that function-case lookup always succeeds at run time.

As is standard [Wright and Felleisen 1994], we prove type soundness via a progress theorem and a type preservation theorem. The progress theorem says that a well-typed expression can always take a step in the dynamic semantics:

Theorem (Progress). If $\vdash E: \tau$ and $E$ is not a value, then there exists $E^{\prime}$ such that $E \longrightarrow E^{\prime}$.

${ }^{8}$ Technically, the version of rules CONCRETE and SuBExT in the static semantics should access the signature table rather than the structure table. It is clear by inspection that the rules do not access anything from a structure that is not also available in its principal signature.

ACM Transactions on Programming Languages and Systems, Vol. 26, No. 5, September 2004. 


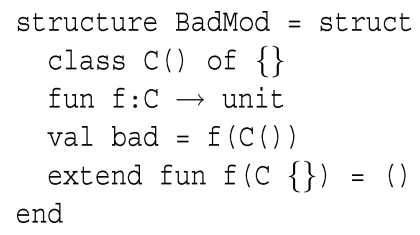

Fig. 20. Value declarations and modular ITC.

The type preservation theorem says that evaluation preserves welltypedness:

Theorem (Type Preservation). If $\vdash E: \tau$ and $E \longrightarrow E^{\prime}$, then there exists $\tau^{\prime}$ such that $\vdash E^{\prime}: \tau^{\prime}$ and $\tau^{\prime} \leq \tau$.

The proofs of these two theorems are provided in Appendix A. Proving progress requires reasoning about modular ITC, in order to show that function applications can always make progress. The key lemma says that a most-specific applicable function case exists for each type-correct function application:

Lemma. If $\vdash F v: \tau_{2} \rightarrow \tau$ and $\vdash v: \tau_{2}^{\prime}$ and $\tau_{2}^{\prime} \leq \tau_{2}$, then there exist $\rho$ and $E$ such that most-specific-case-for $(F v, v)=(\rho, E)$.

Proving type preservation is relatively straightforward, as it is completely independent of modular ITC.

\section{ML-STYLE MODULES}

This section discusses the interaction of EML with an ML-style module system, including structures, signatures, and functors [MacQueen 1984; Milner et al. 1997]. We describe the problems that can arise for modular ITC in this context and sketch some possible solutions.

\subsection{Structures}

Thus far we have assumed that EmL structures contain only a sequence of class, function, and function case declarations. EmL structures should also accommodate the ordinary ML declarations. These include the ability to bind a name to a value, provide a synonym for a type, declare an exception, and declare an inner structure. The latter three kinds of declarations can be straightforwardly incorporated, but special care is needed to handle value declarations. Figure 20 shows an example of the problems that can occur. As presented so far, ITC on BadMod succeeds, because function $f$ has an appropriate case for C. However, at run time a match nonexhaustive error occurs when the val declaration is executed, because f's function case has not yet been declared.

There are several approaches to handling this problem. One solution would be to adopt a two-pass style of structure evaluation. The first pass would evaluate all of the declarations except the value declarations, and the second pass would evaluate the value declarations. In Figure 20, this semantics would ensure that $f$ 's function case is declared before $f$ is invoked. An alternative approach would be to make the unit of modularity used in the ITC requirements more fine-grained than an entire structure, with val declarations forming the 


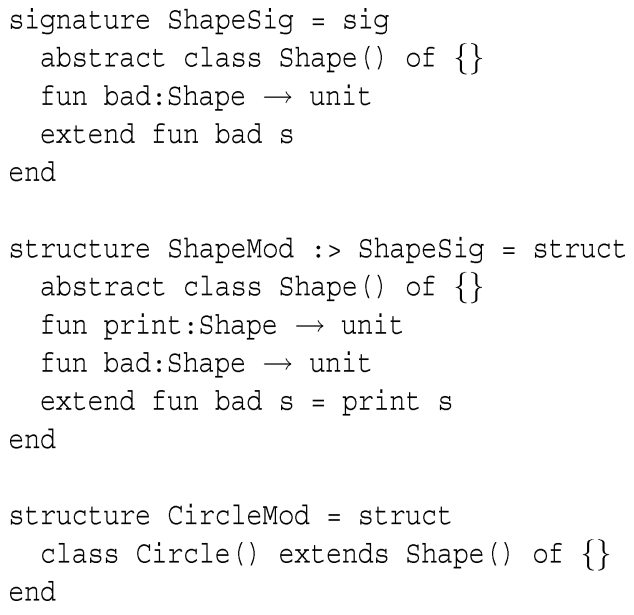

Fig. 21. Signature ascription and modular ITC.

boundaries of these units. For example, BadMod would consist of two units, one of which contains the first two declarations and the other containing the last declaration. When ITC is performed on the first unit, the incompleteness of $f$ for $C$ would result in a static error. Our prototype EmL interpreter uses this approach.

\subsection{Signature Ascription}

By default, clients of an ML structure $S n$ access its components through the view provided by $S n$ 's principal signature. Information hiding in ML is achieved by explicitly ascribing a signature to a structure. Let metavariable $S i g N$ range over signature names. Clients of a structure

$$
\text { structure } S n:>\operatorname{SigN}=\text { struct } \ldots \text { end }
$$

may only access $S n$ 's components through the view provided by the signature SigN. ${ }^{9}$ The ascribed signature may include less information than the structure's principal signature. For example, in ML an ascribed signature may omit specifications for some of the structure's declarations, making them inaccessible to clients.

Signature ascription for EML provides forms of OO-style encapsulation. For example, classes, functions, and function cases can be hidden from clients, making them private to their enclosing structure. However, these declarations cannot be hidden arbitrarily, or else modular ITC would become unsound. Figure 21 shows a simple example of the problems that can occur. ShapeMod creates the abstract Shape class and two associated functions, print and bad. ITC in ShapeMod succeeds vacuously for both functions since Shape is abstract. Because ShapeSig is ascribed to ShapeMod, print is hidden from ShapeMod's clients, who therefore cannot perform ITC on print. ITC succeeds for CircleMod, because bad has a

\footnotetext{
${ }^{9}$ Standard ML includes two kinds of ascription: transparent and opaque. Transparent ascription can be desugared into opaque ascription, so we focus exclusively on the latter form.
}

ACM Transactions on Programming Languages and Systems, Vol. 26, No. 5, September 2004. 
case handling Circles. If bad is ever invoked with a Circle instance, however, print will be invoked, causing a match nonexhaustive error.

The example is purposely similar to the print example in Figure 7. In that case, the global-default requirement ensures that the problem is modularly detected. Intuitively, the modular ITC problems of signature ascription can be solved in the same way: a set of declarations can be safely hidden if that set could have been declared in a separate structure that passes modular ITC [Millstein and Chambers 2002]. The print function in Figure 21 does not satisfy this condition. If print were declared in its own structure, the modular requirements would force the existence of a global default case for print, since print would now be an external function. If print had such a case, then the function (and that case) could be hidden via signature ascription, and the problem for Circle would be resolved.

Aside from hiding entire declarations, it is useful to hide properties of a declaration. Several properties of classes may be hidden. First, any subset of a class's instance variables may be hidden. This hiding does not preclude clients from creating instances of the class, because instances are created only by invoking constructors, and neither the class's constructor nor any constructor arguments have been hidden. As mentioned in Section 4, instance variables are scoped-the name of the structure declaring an instance variable is implicitly part of the name of the instance variable. Therefore, there is no conflict if a subclass in a new module creates an instance variable of the same name as a hidden one in the superclass. Second, a concrete class can be viewed as an abstract one, thereby disallowing clients from instantiating the class. The globaland local-default requirements ensure that clients will provide the appropriate default cases in the face of these supposedly abstract classes. Treating a concrete class as abstract could be useful, for example, to enforce the singleton pattern [Gamma et al. 1995], in which a class has a single instance.

Finally, a signature can declare a class $C$ sealed [Shalit 1997], which hides $C$ 's extensibility: classes declared outside of $C$ 's structure may not directly subclass from $C$. This construct can be used to faithfully model ML-style (nonextensible) datatypes. For example, if SetMod in Figure 1 were ascribed to a signature that specified Set, ListSet, and CListSet as sealed, then other structures would be disallowed from declaring new (direct or indirect) subclasses of Set. In that case, sets form a sealed hierarchy. As discussed in Section 3.3, functions in ML can safely omit (local and global) default cases, because datatypes are nonextensible. EML similarly need not enforce the global- and local-default requirements for functions on sealed hierarchies. For example, if the set hierarchy were sealed, then UnionMod of Figure 2 could safely omit the first union case, which is the global default. Because there would never exist concrete subclasses of Set other than ListSet and CListSet, the second union case in UnionMod would be sufficient to ensure exhaustiveness.

Hiding inheritance properties of classes is more problematic. It would be useful for a signature to expose only a transitive, rather than the direct, superclass of a class. This ability would reduce the dependence of clients on a class's particular implementation. Unfortunately, this flexibility makes modular ITC unsound. For example, a client of two classes $C$ and $C^{\prime}$ can write ambiguous 


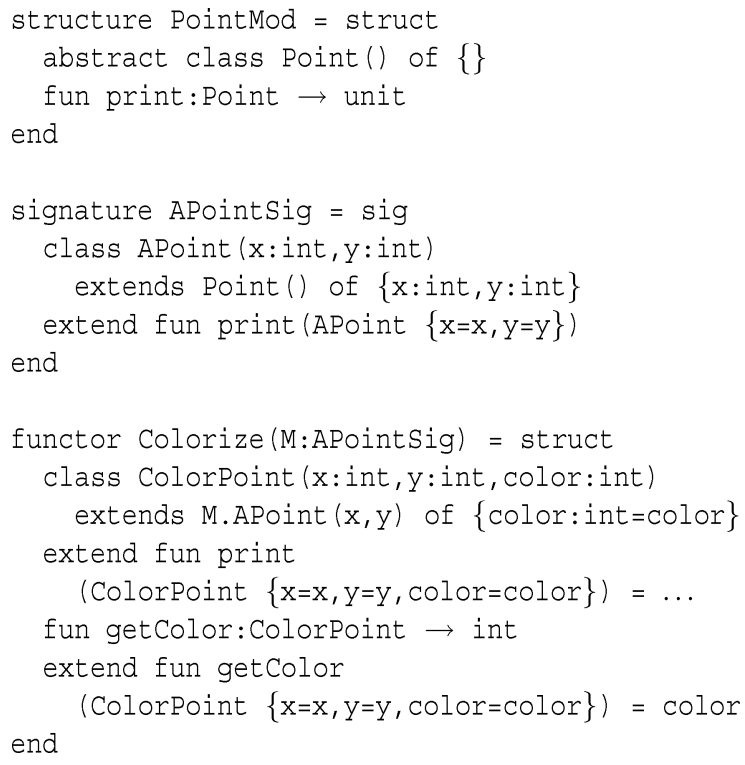

Fig. 22. Encoding mixins with EML functors.

function cases that appear to be unambiguous if the fact that $C$ subclasses $C^{\prime}$ is hidden. It would similarly be useful to ascribe an ML-style type specification to a class declaration, possibly augmenting the specification with partial revelations [Nelson 1991] to reveal some of the class's underlying structure.

Finally, a function may be sealed by ascribing an ordinary ML-style value specification to the function and its cases. For example, union and its two cases in Figure 2 could be represented in a signature by the specification val union $:$ (Set * Set) $\rightarrow$ Set. Clients can still invoke the sealed union function, but its extensibility is hidden (and the \# mark is no longer necessary): clients may not add new cases to union and do not perform ITC on it. In this way, function sealing allows us to model ML-style (nonextensible) functions. Function sealing is allowed under the same circumstances that the function and its cases may be hidden, thereby ensuring that the sealed function is exhaustive and unambiguous.

\subsection{Functors}

Standard ML supports functors, which are structures parameterized by other structures. In the presence of EmL's features, functors can provide a great deal of flexibility. Figure 22 illustrates some of the idioms that would be useful to express. The PointMod structure contains a Point base class with an associated print function. The Colorize functor implements a form of mixin [Bracha and Cook 1990; Findler and Flatt 1998; Flatt et al. 1998], which is a class parameterized by its superclass. The functor can be instantiated by applying it to any structure that conforms to the APointSig signature, thereby creating a colored version of that structure's Point subclass. An overriding case for the existing print function is given, in order to print colored points specially. The functor 


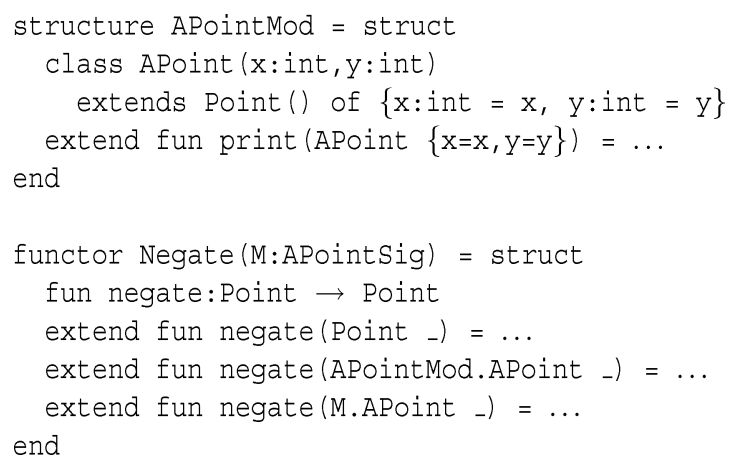

Fig. 23. Three-valued modular ITC of functor bodies.

also introduces a new function for accessing the color of a colored point, with an associated case.

In ML, each functor body can be safely typechecked once, given only the signature of the argument structure. EML should similarly perform modular ITC once on a functor body, guaranteeing exhaustiveness and unambiguity of all relevant functions, no matter how the functor will be instantiated. The major challenge for modular ITC of functors like Colorize is the fact that the identities of some classes, for example M. APoint, are unknown. Instead only partial information is known about the relationship between M. APoint and other classes. While others have investigated the integration of functors (or related forms of parameterized modules) with classes (e.g. [Fisher and Reppy 1999; Ancona and Zucca 2001; McDirmid et al. 2001]), the interaction of functors with EML's generalization of $\mathrm{OO}$ and functional dispatching semantics has not been considered previously.

A possible approach to conservatively performing ITC in the presence of partial information in EML is to generalize the subclass relation in the static semantics to be three-valued, saying "don't know" when the partial class hierarchy information is inconclusive. The pattern specificity relation is then also generalized to be three-valued, making use of the generalized subclass relation. Last, modular ITC is modified to be conservative with respect to threevalued subclassing and pattern specificity. In exhaustiveness checking, a local default should be required for any class that may subclass an available function's owner. The generated (local or global) default pattern must be at least as specific as some function case's pattern. In ambiguity checking, all tests for pattern congruence and disjointness should succeed only if the given patterns must be congruent and disjoint, respectively. Similarly, the intersection of two patterns should result in an exact intersection pattern. If the two patterns are neither definitely disjoint nor definitely intersecting, then the associated cases are conservatively considered ambiguous.

As an example, consider three-valued ITC on negate in Negate of Figure 23. The first case is definitely a global default, so negate is exhaustive. The first two cases are found to be unambiguous as usual. The first and third cases are similarly found to be unambiguous: even though the identity of M. APoint is not 
known, from APointSig in Figure 22 it is clear that M. APoint strictly subclasses Point, and this is enough information to compute the exact intersection of the two patterns. Finally, the second and third cases are found to be ambiguous: their patterns are neither definitely disjoint nor definitely intersecting. Therefore ITC on negate fails. Indeed, if Negate is ever instantiated with APointMod, the resulting negate function will be ambiguous for APoint.

The restrictions on signature ascription described earlier can severely limit the reusability of functors. For example, the Colorize and Negate functors can only be instantiated with a class APoint that is a direct subclass of Point, rather than an indirect (transitive) one. Also, APoint's module must contain a print case with the pattern specified in APointSig. While relaxing these restrictions would increase functor reusability, it would also force ITC on functor bodies to become much more conservative, in order to account for the new expressiveness of clients. The relaxation could therefore reduce the overall benefit of functors by requiring the type system to disallow too many of them.

A pragmatic way to avoid the restrictions on signature ascription could be to move some of the burden of ITC to clients of the functor. In the limit, EML would perform modular ITC once per instantiation of the functor, on the structure resulting from the instantiation. At the point of instantiation, all the identities of classes and functions in the functor's argument would be known, so ordinary modular ITC as described in Section 3.3 would suffice. It is possible that in practice most of ITC could still be performed on the functor body in isolation, with only a few additional checks performed per instantiation. Such a scheme would provide early feedback about a functor's type correctness while still safely supporting the desired expressiveness.

\section{RELATED WORK}

In previous work we defined Dubious [Millstein and Chambers 1999, 2002], an expressive multimethod-based $\mathrm{OO}$ calculus, along with requirements for modular typechecking of Dubious programs. Dubious was then used as the foundation for our work on MultiJava [Clifton et al. 2000], an extension to Java that supports multimethods as well as the addition of new operations to existing classes. MultiJava illustrates how Dubious's expressiveness and modular typechecking requirements can underlie an extension to a traditional OO language, and EML does the same for a traditional functional language. In particular, in EmL we have adapted Dubious's requirements to support modular typechecking of extensible datatypes and functions in an ML-like setting. EML also improves on Dubious's theoretical foundations in several ways. First, we have significantly simplified both the informal and formal presentations of the modularity requirements. Second, the notion of modularity in EML is stronger than that of Dubious: an EML module requires access to less of the program to soundly perform ITC than does a Dubious module. Finally, Dubious does not address modular typechecking in the face of pattern matching, parametric polymorphism, or ML-style modules.

OML [Reppy and Riecke 1996] and $\mathrm{ML}_{\leq}$[Bourdoncle and Merz 1997] were described earlier. Zenger and Odersky [2001] describe an extensible datatype 
mechanism in the context of an OO language. Extending a datatype has the effect of creating a new datatype that subtypes from the original one. To ensure exhaustiveness in the presence of datatype extension, all functions on extensible datatypes must include a global default case, while EmL often requires only local defaults. Because Zenger's functions are not extensible, if new data variants require overriding function cases, a new function must be created that inherits the existing function cases and clients must be modified to invoke the new function. Like OML, Zenger's language includes both OO-style methods and ML-style functions. Garrigue shows how to use polymorphic variants, which are variants defined independent of any particular datatype, to obtain both modular data-variant and function extensibility in ML [Garrigue 2000]. As in Zenger's language, functions are not extensible, so existing clients must be modified as new variants with overriding function cases are introduced. Unlike EML, polymorphic variants preserve ML-style type inference.

The mixin modules of Duggan and Sourelis [1996] allow datatype and function declarations to be split across multiple modules, thereby providing a form of extensible datatypes and functions. ${ }^{10}$ Mixin modules must be explicitly composed with one another and "closed" to form an ordinary ML module containing nonextensible datatypes and functions. Therefore, adding a new mixin module that extends an existing datatype or function requires modifying existing code to explicitly include the new mixin module in the composition. In contrast, EML datatypes and functions may be extended by new modules without modifying existing code. Mixin modules are also not hierarchical and do not support subtyping. Follow-on work [Duggan and Techaubol 2001] incorporates mixin modules into a traditional class-based $\mathrm{OO}$ language. A distinction is made between class-based and mixin-based objects; mixin-based objects do not support subtyping. Mixin-based objects support a form of type specialization for methods, which EmL lacks.

Jiazzi [McDirmid et al. 2001] is an extension to Java based on units [Findler and Flatt 1998; Flatt and Felleisen 1998], a form of parameterized module with recursive linking. The authors show how to encode an open class pattern in Jiazzi, whereby a module imports a class and exports a version of that class modified to contain a new method or field. In this way, Jiazzi supports adding both new subclasses and new methods to existing classes. Similar to mixin modules, the final version of a class must be explicitly created by composing the relevant modules.

Work on predicate dispatching [Ernst et al. 1998] describes ITC for patterns that are more general than those in EML, including conjunctions, disjunctions, and negations of arbitrary predicates in the host language. An OO-style dispatching semantics is used, with predicate implication as the specificity relation among patterns, and functions are extensible. However, the ITC algorithm is nonmodular, requiring access to the entire program.

${ }^{10}$ Other proposals that also use the name "mixin modules" [Ancona and Zucca 2002; Hirschowitz and Leroy 2002] lack extensible datatypes and functions and are not directly related to our work. 


\section{CONCLUSIONS AND FUTURE WORK}

We described Extensible ML, an ML-like language that supports hierarchical, extensible datatypes and functions. Such constructs allow for the easy addition of both new data variants and new operations to existing abstractions, resolving a longstanding tension between the functional and object-oriented styles. At the same time, EML retains completely modular typechecking of function implementations. This contrasts with previous languages based on extensible datatypes and functions, which require link-time checks to ensure type safety. We have formalized EmL in Mini-EmL and proven its type system sound.

There are several directions for future work. First, Section 2.5 discussed future work related to EmL's polymorphic type system. Second, more work is needed to integrate EML with ML-style modules, particularly functors. Section 5 sketched some of the challenges and solutions, but additional study and experience are necessary to find practical requirements that balance expressiveness and modular typechecking. Third, EmL's extensibility is currently second class: only statically known classes and functions may be extended. Allowing lexically nested classes and functions to be created and extended would enhance EmL's expressiveness and would better integrate its constructs with the ML style. A form of linear types [Wadler 1990] could possibly be used to statically track classes and functions for the purpose of ITC. Finally, it would be useful to investigate practical compilation techniques for EmL's extensible datatypes and functions. These techniques can likely build on efficient implementation strategies for multimethod and predicate dispatch [Chambers and Chen 1999].

\section{APPENDIX}

\section{A. TYPE SOUNDNESS FOR MINI-EML}

This appendix provides the proof of type soundness for Mini-EmL. As discussed in Section 4.4, progress and type preservation theorems are proven. The first section below contains a proof of the progress theorem and the key technical lemmas that it depends upon. The second section does the same for the type preservation theorem. Both theorems also rely on several basic facts about Mini-EmL, which are stated in the final section.

As in the formal dynamic and static semantics, the proof assumes a fixed structure table $S T$ satisfying the two sanity conditions given in Section 4 and a fixed signature table SigT containing the principal signatures of the structures in $S T$. Also as in the formal rules, it is assumed that $S$ OK holds for each structure $S$ in $S T$.

\section{A.1 Progress}

The progress theorem is straightforward except for the case when $E$ is a function application. That case follows easily from lemma 1, which is given below.

Theorem 1 (Progress). If $\vdash E: \tau$ and $E$ is not a value, then there exists an $E^{\prime}$ such that $E \longrightarrow E^{\prime}$. 
Proof. By (strong) induction on the depth of the derivation of $\vdash E: \tau$. Case analysis of the last rule used in the derivation.

- Case T-ID. Then $E=I$ and $(I, \tau) \in\{\}$, so we have a contradiction. Therefore this rule could not be the last rule used in the derivation.

- Case T-New. Then $E=C t(\bar{E})$ and $C t=(\bar{\tau} S n . C n)$ and \{\}$; \bullet \vdash C t(\bar{E})$ OK and concrete $(S n . C n)$. Therefore by Lemma 32 , there exist $\overline{V_{1}}$ and $\overline{E_{1}}$ such that $\operatorname{rep}(C t(\bar{E}))=\left\{\overline{V_{1}}=\overline{E_{1}}\right\}$. Then by E-New we have $E \longrightarrow C t\left\{\overline{V_{1}}=\overline{E_{1}}\right\}$.

- Case T-Rep. Then $E=C t\left\{V_{1}=E_{1}, \ldots, V_{k}=E_{k}\right\}$ and for all $1 \leq i \leq k$ we have $\vdash E_{i}: \tau_{i}$ for some $\tau_{i}$. We have two subcases:

-For all $1 \leq i \leq k, E_{i}$ is a value. Then $E$ is a value, contradicting our assumption.

-There exists some $j$ such that $1 \leq j \leq k$ and $E_{j}$ is not a value. Without loss of generality, let $j$ be the smallest integer satisfying this condition, so for all $1 \leq q<j$ we have that $E_{q}$ is a value. By induction, there exists an $E_{j}^{\prime}$ such that $E_{j} \longrightarrow E_{j}^{\prime}$. Therefore by E-REP we have $C t\left\{V_{1}=E_{1}, \ldots, V_{k}=\right.$ $\left.E_{k}\right\} \longrightarrow C t\left\{V_{1}=E_{1}, \ldots, V_{j-1}=E_{j-1}, V_{j}=E_{j}^{\prime}, V_{j+1}=E_{j+1}, \ldots, V_{k}=\right.$ $\left.E_{k}\right\}$.

- Case T-Fun. Then $E=\bar{\tau}$ Sn.Fn. Then $E$ is a value, contradicting our assumption.

- Case T-Tup. Then $E=\left(E_{1}, \ldots, E_{k}\right)$ and $\tau=\tau_{1} * \cdots * \tau_{k}$ and for all $1 \leq i \leq k$ we have $\vdash E_{i}: \tau_{i}$. We have two subcases:

-For all $1 \leq i \leq k, E_{i}$ is a value. Then $E$ is a value, contradicting our assumption.

- There exists some $j$ such that $1 \leq j \leq k$ and $E_{j}$ is not a value. Without loss of generality, let $j$ be the smallest integer satisfying this condition, so for all $1 \leq q<j$ we have that $E_{q}$ is a value. By induction, there exists an $E_{j}^{\prime}$ such that $E_{j} \longrightarrow E_{j}^{\prime}$. Therefore by E-Tup we have $\left(E_{1}, \ldots, E_{k}\right) \longrightarrow$ $\left(E_{1}, \ldots, E_{j-1}, E_{j}^{\prime}, E_{j+1}, \ldots, E_{k}\right)$.

- Case T-App. Then $E=E_{1} E_{2}$ and $\vdash E_{1}: \tau_{2} \rightarrow \tau$ and $\vdash E_{2}: \tau_{2}^{\prime}$ and $\tau_{2}^{\prime} \leq \tau_{2}$. We have three subcases:

$-E_{1}$ is not a value. Then by induction, there exists an $E_{1}^{\prime}$ such that $E_{1} \longrightarrow$ $E_{1}^{\prime}$. Therefore by E-App 1 we have $E_{1} E_{2} \longrightarrow E_{1}^{\prime} E_{2}$.

$-E_{1}$ is a value, but $E_{2}$ is not a value. Then by induction, there exists an $E_{2}^{\prime}$ such that $E_{2} \longrightarrow E_{2}^{\prime}$. Therefore by E-ApP2 we have $E_{1} E_{2} \longrightarrow E_{1} E_{2}^{\prime}$.

-Both $E_{1}$ and $E_{2}$ are values. Since $\vdash E_{1}: \tau_{2} \rightarrow \tau$ and $E_{1}$ is a value, the last rule in the derivation of $\vdash E_{1}: \tau_{2} \rightarrow \tau$ must be T-Fun, so $E_{1}$ has the form $F v$. Therefore by Lemma 1 we have that there exist $\rho_{0}$ and $E_{0}$ such that most-specific-case-for $\left(F v, E_{2}\right)=\left(\rho_{0}, E_{0}\right)$. Let $\rho_{0}=\{(\bar{I}, \bar{v})\}$. Then by E-AppRED we have $F v E_{2} \longrightarrow[\bar{I} \mapsto \bar{v}] E_{0}$.

This lemma says that a most-specific applicable function case exists for each type-correct function application. It follows easily from Lemmas 2 and 7, which say that all functions are exhaustive and unambiguous, respectively.

Lemma 1. If $\vdash F v: \tau_{2} \rightarrow \tau$ and $\vdash v: \tau_{2}^{\prime}$ and $\tau_{2}^{\prime} \leq \tau_{2}$ then there exist $\rho$ and $E$ such that most-specific-case-for $(F v, v)=(\rho, E)$. 
Proof. Let $F v=(\bar{\tau} F)$. By Lemma 2, there exists some $S n \in \operatorname{dom}(\operatorname{Sig} T)$, some (extend $\operatorname{fun}_{M n} \bar{\alpha} F$ Pat) $\in \operatorname{Sig} T(S n)$, and some environment $\rho_{0}$ such that $\operatorname{match}(v, P a t)=\rho_{0}$. Then by Lemma 7 there exists some $S n^{\prime} \in \operatorname{dom}(\operatorname{Sig} T)$, some (extend $\left.\operatorname{fun}_{M n^{\prime}} \overline{\alpha_{1}} F P a t^{\prime}\right) \in \operatorname{Sig} T\left(S n^{\prime}\right)$, and some $\rho^{\prime} \operatorname{such}$ that $\operatorname{match}\left(v, \operatorname{Pat}^{\prime}\right)=$ $\rho^{\prime}$ and $\forall S n^{\prime \prime} \in \operatorname{dom}(\operatorname{Sig} T) . \forall\left(\right.$ extend $\left.\operatorname{fun}_{M n^{\prime \prime}} \overline{\alpha_{2}} F P a t^{\prime \prime}\right) \in \operatorname{Sig} T\left(\operatorname{Sn}^{\prime \prime}\right) . \forall \rho^{\prime \prime}$. $\left(\left(\operatorname{match}\left(v, P a t^{\prime \prime}\right)=\rho^{\prime \prime} \wedge S n^{\prime} . M n^{\prime} \neq S n^{\prime \prime} . M n^{\prime \prime}\right) \Rightarrow P a t^{\prime}<P a t^{\prime \prime}\right)$.

By the definition of $\operatorname{Sig} T$ we have that $S n^{\prime} \in \operatorname{dom}(S T)$ and there exists some $E^{\prime}$ such that (extend $\left.f_{n_{M n^{\prime}}} \overline{\alpha_{1}} F P a t^{\prime}\right) \in S T\left(S n^{\prime}\right)$ and $\forall S n^{\prime \prime} \in$ $\operatorname{dom}(S T) . \forall\left(\right.$ extend $\left.\operatorname{fun}_{M n^{\prime \prime}} \overline{\alpha_{2}} F P a t^{\prime \prime}=E^{\prime \prime}\right) \in S T\left(S n^{\prime \prime}\right) \cdot \forall \rho^{\prime \prime} .\left(\left(\operatorname{match}\left(v, P a t^{\prime \prime}\right)=\right.\right.$ $\left.\left.\rho^{\prime \prime} \wedge S n^{\prime} . M n^{\prime} \neq S n^{\prime \prime} . M n^{\prime \prime}\right) \Rightarrow P a t^{\prime}<P a t^{\prime \prime}\right)$.

Since $\vdash F v: \tau_{2} \rightarrow \tau$, by T-Fun we have $F=S n_{0} \cdot F n_{0}$ and (fun $\overline{\alpha_{0}} F n_{0}: M t_{0} \rightarrow$ $\left.\tau_{0}\right) \in \operatorname{Sig} T\left(S n_{0}\right)$ and $\left|\overline{\alpha_{0}}\right|=|\bar{\tau}|$. Since (extend $\left.\operatorname{fun}_{M n^{\prime}} \overline{\alpha_{1}} F P a t^{\prime}\right) \in \operatorname{Sig} T\left(S n^{\prime}\right)$, by CASEOK we have $\left|\overline{\alpha_{1}}\right|=\left|\overline{\alpha_{0}}\right|$. Therefore we have $\left|\overline{\alpha_{1}}\right|=|\bar{\tau}|$. Then by Lookup there exist $\rho$ and $E$ such that most-specific-case-for $((\bar{\tau} F), v)=(\rho, E)$.

A.1.1 Exhaustiveness. These lemmas prove that all functions are exhaustive. They make use of the notion of the owner of a value $v$ with respect to a marked type $M t$. Intuitively, $v$ 's owner is the class in $v$ located at the owner position as specified by $M t$. It is defined via the following two inference rules:

$$
\begin{gathered}
\text { owner }(M t, v)=C \quad \operatorname{owner}\left(M t, v_{i}\right)=C \\
\frac{\operatorname{owner}\left(\tau_{1} * \ldots * \tau_{i-1} * M t * \tau_{i+1} * \ldots * \tau_{k}\left(v_{1}, \ldots, v_{k}\right)\right)=C}{\operatorname{owner}(\# C t,(\bar{\tau} C)\{\bar{V}=\bar{v}\})=C} \text { OwnERTUPVAL } \\
\frac{\text { OwnERINSTANCE }}{}
\end{gathered}
$$

This is the main exhaustiveness lemma, which says that every type-correct argument value for a function has at least one applicable function case. Its proof follows from the global- and local-default requirements. If the function has a global default case, then the result follows from the definition of a global default. Otherwise, the function must be internal. Then there must be a local default case for each type-correct argument value's owner, and the result follows from the definition of a local default case.

Lemma 2 (Exhaustiveness). If $\vdash(\bar{\tau} F): \tau_{2} \rightarrow \tau$ and $\vdash v: \tau_{2}^{\prime}$ and $\tau_{2}^{\prime} \leq \tau_{2}$, then there exist some $S n \in \operatorname{dom}(\operatorname{SigT})$, some (extend $\left.\operatorname{fun}_{\mathrm{Mn}} \overline{\alpha_{1}} F P a t\right) \in \operatorname{Sig} T(S n)$, and some environment $\rho$ such that match $(v$, Pat $)=\rho$.

Proof. Since $\vdash(\bar{\tau} F): \tau_{2} \rightarrow \tau$, by T-Fun we have $F=S n^{\prime} . F n$ and (fun $\bar{\alpha}$ $\left.F n: M t \rightarrow \tau_{0}\right) \in \operatorname{Sig} T\left(S n^{\prime}\right)$ and $|\bar{\alpha}|=|\bar{\tau}|$ and $\tau_{2} \rightarrow \tau=[\bar{\alpha} \mapsto \bar{\tau}]\left(\hat{M} t \rightarrow \tau_{0}\right)$. By the definition of $\operatorname{Sig} T$, also (fun $\left.\bar{\alpha} F n: M t \rightarrow \tau_{0}\right) \in S T\left(S n^{\prime}\right)$. Let $S T\left(S n^{\prime}\right)=$ (structure $S n^{\prime}=$ struct $\bar{D}$ end). Then by $\operatorname{StructOK}$ we have $\overline{S n} \subseteq \operatorname{dom}(\operatorname{SigT})$ and $\overline{S n} \vdash\left(\right.$ fun $\left.\bar{\alpha} F n: M t \rightarrow \tau_{0}\right)$ OK in $S n^{\prime}$, so by FunOK we have that owner $(M t)=$ $S n^{\prime \prime}$.Cn. Then by Lemma 3 there exists some class $C$ such that owner $(M t, v)=$ $C$ and concrete $(C)$ and $C \leq S n^{\prime \prime} . C n$. Also by FunOK we have either $\overline{S n} \vdash$ $F$ has-gdefault or $S n^{\prime}=S n^{\prime \prime}$. We consider these cases separately. 
- Case $\overline{S n} \vdash F$ has-gdefault. By GDefault we have owner $(F)=C^{\prime}$ and $\overline{S n} \vdash$ $F$ has-default-for $C^{\prime}$. By OwnerFun, $C^{\prime}=S n^{\prime \prime} . C n$. Then by Lemma 4 there exists some $S n \in \overline{S n}$, some (extend $\operatorname{fun}_{M n} \overline{\alpha_{1}} F$ Pat) $\in \operatorname{Sig} T(S n)$, and some environment $\rho$ such that $\operatorname{match}(v, P a t)=\rho$. Since $\overline{\operatorname{Sn}} \subseteq \operatorname{dom}(\operatorname{Sig} T)$, we have $S n \in \operatorname{dom}(\operatorname{Sig} T)$ and the result is shown.

- Case $S n^{\prime}=S n^{\prime \prime}$. Let $C=S n_{0} \cdot C n_{0}$. Since concrete $(C)$, by ConCRETe we have (class $\left.\overline{\alpha_{0}} C n_{0} \ldots\right) \in S T\left(S n_{0}\right)$. Let $S T\left(S n_{0}\right)=$ (structure $S n_{0}=$ struct $\overline{D_{0}}$ end). Then by StructOK we have $\overline{S n_{0}} \subseteq \operatorname{dom}(\operatorname{SigT})$ and $\overline{S n_{0}} \vdash$ class $\overline{\alpha_{0}} C n_{0} \ldots$ OK in $S n_{0}$, so by ClassOK we have concrete $(C) \Rightarrow \overline{S n_{0}} \vdash$ funs-have-ldefault-for $C$. Since concrete $(C)$ holds, we have $\overline{S n_{0}} \vdash$ funs-have-ldefault-for $C$.

Also by ClassOK we have $\overline{S n_{0}} \vdash C$ ITCTransUses. Since $C \leq S n^{\prime \prime} . C n$ and $S n^{\prime}=S n^{\prime \prime}$, by Lemma 37 we have $S n^{\prime} \in \overline{S n_{0}}$.

Since $F=S n^{\prime} . F n$ and $S n^{\prime} \in \overline{S n_{0}}$, by FunITCUses we have $\overline{S n_{0}} \vdash$ $F$ ITCUses. Since (fun $\left.\bar{\alpha} F n: M t \rightarrow \tau_{0}\right) \in \operatorname{Sig} T\left(S n^{\prime}\right)$ and owner $(M t)=$ $S n^{\prime}$.Cn, by OwnerFun we have owner $(F)=S n^{\prime}$.Cn. Also, we showed above that $C \leq S n^{\prime}$.Cn. Therefore, since $\overline{S n_{0}} \vdash$ funs-have-ldefault-for $C$, by LDEFAUlT we have $\overline{S n_{0}} \vdash F$ has-default-for $C$. By SubReF $C \leq C$, so by Lemma 4 there exists some $S n \in \overline{S n_{0}}$, some (extend fun $\left._{M n} \overline{\alpha_{1}} S n . F n P a t\right) \in \operatorname{SigT}(S n)$, and some environment $\rho$ such that $\operatorname{match}(v, P a t)=\rho$. Since $\overline{S n_{0}} \subseteq \operatorname{dom}(\operatorname{Sig} T)$, we have $S n \in \operatorname{dom}(\operatorname{Sig} T)$, and the result is shown.

This lemma says that a value conforming to a marked type has a well-defined owner (with respect to that marked type), which is a subclass of the marked type's owner.

Lemma 3. If $\vdash v: \tau^{\prime}$ and $\tau^{\prime} \leq \tau$ and $\tau=[\bar{\alpha} \mapsto \bar{\tau}] \hat{M t}$ and owner $(M t)=C^{\prime}$, then there exists some class $C$ such that owner $(M t, v)=C$ and concrete $(C)$ and $C \leq C^{\prime}$.

Proof. By induction on the depth of the derivation of $\vdash v: \tau^{\prime}$. Case analysis of the last rule used in the derivation.

- Case T-Rep. Then $v$ has the form $\left(\overline{\tau_{0}} C\right)\{\bar{V}=\bar{v}\}$ and $\tau^{\prime}=\left(\overline{\tau_{0}} C\right)$ and concrete $(C)$. Since $\tau^{\prime} \leq \tau$, by Lemma $13 \tau$ has the form $\left(\overline{\tau_{1}} C^{\prime \prime}\right)$. Since $\tau=[\bar{\alpha} \mapsto \bar{\tau}] \hat{M} t, \hat{M} t$ has the form $\left(\overline{\tau_{2}} C^{\prime \prime}\right)$, and by the grammar for marked types $M t$ must be \#( $\left.\overline{\tau_{2}} C^{\prime \prime}\right)$. Then by OwneRInSTANCE we have owner $\left(\#\left(\overline{\tau_{2}} C^{\prime \prime}\right),\left(\overline{\tau_{0}} C\right)\{\bar{V}=\bar{v}\}\right)=C$. We're given $\tau^{\prime} \leq \tau$, so by lemma 15 we have $C \leq C^{\prime \prime}$. Since owner $(M t)=C^{\prime}$, by OwnerClass we have $C^{\prime}=C^{\prime \prime}$, so $C \leq C^{\prime}$.

- Case T-Fun. Then $v$ has the form $\left(\overline{\tau_{0}} F\right)$ and $\tau^{\prime}$ has the form $\tau_{1} \rightarrow \tau_{2}$. Therefore by Lemma $18 \tau$ has the form $\tau_{1}^{\prime} \rightarrow \tau_{2}^{\prime}$. Since $\tau=[\bar{\alpha} \mapsto \bar{\tau}] \hat{M} t, \hat{M} t$ has the form $\tau_{1}^{\prime \prime} \rightarrow \tau_{2}^{\prime \prime}$, but this contradicts the grammar of marked types. Therefore, T-FuN cannot be the last rule in the derivation.

- Case T-Tup: Then $v$ has the form $\left(v_{1}, \ldots, v_{k}\right)$ and $\tau^{\prime}$ has the form $\tau_{1}^{\prime} * \cdots * \tau_{k}^{\prime}$ and for all $1 \leq j \leq k$ we have $\vdash v_{j}: \tau_{j}^{\prime}$. Therefore by Lemma $20 \tau$ has the form $\tau_{1} * \cdots * \tau_{k}$, where for all $1 \leq j \leq k$ we have $\tau_{j}^{\prime} \leq \tau_{j}$. Since $\tau=[\bar{\alpha} \mapsto \bar{\tau}] \hat{M} t$, $\hat{M} t$ has the form $\tau_{1}^{\prime \prime} * \cdots * \tau_{k}^{\prime \prime}$, and by the grammar for marked types $M t$ is 
$\tau_{1}^{\prime \prime} * \cdots * \tau_{i-1}^{\prime \prime} * M t_{i} * \tau_{i+1}^{\prime \prime} * \cdots * \tau_{k}^{\prime \prime}$, where $\hat{M} t_{i}=\tau_{i}^{\prime \prime}$. We're given owner $(M t)=$ $C^{\prime}$, so by OwnerTup we have owner $\left(M t_{i}\right)=C^{\prime}$.

Therefore we have $\vdash v_{i}: \tau_{i}^{\prime}$ and $\tau_{i}^{\prime} \leq \tau_{i}$ and $\tau_{i}=[\bar{\alpha} \mapsto \bar{\tau}] \hat{M} t_{i}$ and owner $\left(M t_{i}\right)=C^{\prime}$, so by induction there exists $C$ such that owner $\left(M t_{i}, v_{i}\right)=C$ and concrete $(C)$ and $C \leq C^{\prime}$. By OwnerTupVAL we have owner $\left(\tau_{1}^{\prime \prime} * \cdots * \tau_{i-1}^{\prime \prime} *\right.$ $\left.M t_{i} * \tau_{i+1}^{\prime \prime} * \cdots * \tau_{k}^{\prime \prime},\left(v_{1}, \ldots, v_{k}\right)\right)=C$, so the result follows.

This lemma validates the has-default-for judgment: if a function $F$ has a default for class $C$, then $F$ has at least one applicable function case for each type-correct argument value whose owner is a subclass of $C$.

Lemma 4. If $\vdash v: \tau_{2}^{\prime}$ and $\tau_{2}^{\prime} \leq \tau_{2}$ and $\tau_{2}=[\bar{\alpha} \mapsto \bar{\tau}] \hat{M} t$ and (fun $\bar{\alpha}$ $\left.F n: M t \rightarrow \tau_{0}\right) \in \operatorname{SigT}(S n)$ and owner $(M t, v)=C_{0}$ and $C_{0} \leq C$ and $\overline{S n} \vdash$ $S n . F n$ has-default-for $C$, then there exists some $S n^{\prime} \in \overline{S n}$, some (extend fun ${ }_{M n}$ $\overline{\alpha_{1}} \operatorname{Sn} . F n$ Pat $) \in \operatorname{SigT}\left(S^{\prime}\right)$, and some environment $\rho$ such that match $(v, P a t)=$ $\rho$.

Proof. Since $\overline{S n} \vdash S n . F n$ has-default-for $C$, by Default we have defaultPat $(M t, C, d)=P a t^{\prime}$. Therefore by Lemma 5 there exists $\rho^{\prime}$ such that $\operatorname{match}\left(v, P_{a} t^{\prime}\right)=\rho^{\prime}$. Also by Default we have (extend fun $_{M n} \overline{\alpha_{1}}$ Sn.Fn Pat) $\in S i g T\left(S n^{\prime}\right)$ and $P a t^{\prime} \leq P a t$ and $S n^{\prime} \in \overline{S n}$. By Lemma 25 there exists $\rho$ such that $\operatorname{match}(v, P a t)=\rho$, so the result follows.

The next two lemmas validate the defaultPat judgment, by showing that the generated pattern is in fact a (global or local) default pattern: each type-correct argument value matches the generated pattern. The first lemma handles patterns generated with respect to marked types, and the second lemma handles patterns generated with respect to ordinary unmarked types.

Lemma 5. If $\vdash v: \tau^{\prime}$ and $\tau^{\prime} \leq \tau$ and $\tau=[\bar{\alpha} \mapsto \bar{\tau}] \hat{M} t$ and owner $(M t, v)=$ $C_{0}$ and $C_{0} \leq C$ and defaultPat $(M t, C, d)=$ Pat, then there exists $\rho$ such that match $(v$, Pat $)=\rho$.

PRoof. By strong induction on the depth of the derivation of $\operatorname{defaultPat}(M t, C, d)=$ Pat. Case analysis of the last rule in the derivation.

- Case DefZero. Then Pat has the form _, so by E-MatchWild we have $\operatorname{match}\left(v,{ }_{-}\right)=\{\}$.

- Case DefOwnerClassType. Then $M t$ has the form \#( $\left.\bar{\tau}_{1} C^{\prime}\right)$ and Pat has the form $(C\{\bar{V}=\overline{P a t}\})$ and repType $\left(\overline{\tau_{1}} C\right)=\left\{\bar{V}: \overline{\tau^{\prime}}\right\}$ and defaultPat $\left(\overline{\tau^{\prime}}, C, d-1\right)=$ $\overline{P a t}$ and $d>0$. By Lemma 23 we have repType $\left([\bar{\alpha} \mapsto \bar{\tau}] \overline{\tau_{1}} C\right)=[\bar{\alpha} \mapsto \bar{\tau}]\left\{\bar{V}: \overline{\tau^{\prime}}\right\}$. Since owner $\left(\#\left(\bar{\tau}_{1} C^{\prime}\right), v\right)=C_{0}$, by OwNERINSTANCE we have that $v$ is of the form $\left(\overline{\tau_{0}} C_{0}\right)\left\{\overline{V_{2}}=\overline{v_{2}}\right\}$.

Since we're given that $\vdash v: \tau^{\prime}$, by T-REP we have that $\tau^{\prime}=\left(\overline{\tau_{0}} C_{0}\right)$ and - $\vdash\left(\overline{\tau_{0}} C_{0}\right)$ OK and repType $\left(\overline{\tau_{0}} C_{0}\right)=\left\{\overline{V_{2}}: \overline{\tau_{2}}\right\}$ and $\vdash \overline{v_{1}}: \overline{\tau_{2}^{\prime}}$ and $\overline{\tau_{2}^{\prime}} \leq \overline{\tau_{2}}$. We're given that $\tau^{\prime} \leq \tau$, so that means $\left(\overline{\tau_{0}} C_{0}\right) \leq\left([\bar{\alpha} \mapsto \bar{\tau}] \overline{\tau_{1}} C^{\prime}\right)$, and by Lemma 14 we have $\overline{\tau_{0}}=[\bar{\alpha} \mapsto \bar{\tau}] \overline{\tau_{1}}$. Since $C_{0} \leq C$ and $\bullet \vdash\left(\overline{\tau_{0}} C_{0}\right)$ OK, by Lemma 16 we 
have $\left(\overline{\tau_{0}} C_{0}\right) \leq\left(\overline{\tau_{0}} C\right)$. Therefore by Lemma 36 we have $\left\{\overline{V_{2}}: \overline{\tau_{2}}\right\}=\{\bar{V}:[\bar{\alpha} \mapsto$ $\left.\bar{\tau}] \overline{\tau^{\prime}}, \overline{V_{3}}: \overline{\tau_{3}}\right\}$.

Therefore there is some prefix $\overline{v_{4}}$ of $\overline{v_{2}}$ and some prefix $\overline{\tau_{4}}$ of $\overline{\tau_{2}^{\prime}}$ such that $\vdash \overline{v_{4}}: \overline{\tau_{4}}$ and $\overline{\tau_{4}} \leq[\bar{\alpha} \mapsto \bar{\tau}] \overline{\tau^{\prime}}$ and defaultPat $\left(\overline{\tau^{\prime}}, C, d-1\right)=\overline{P a t}$, so by Lemma 6 , there exists $\bar{\rho}$ such that match $\left(\overline{v_{4}}, \overline{P a t}\right)=\bigcup \bar{\rho}$. Finally, we're given $C_{0} \leq C$, so by E-MatchClass we have $\operatorname{match}\left(\left(\overline{\tau_{0}} C_{0}\right)\left\{\overline{V_{2}}=\overline{v_{2}}\right\},(C\{\bar{V}=\overline{P a t}\})\right)=\bigcup \bar{\rho}$.

- Case DefTupType. Then $M t$ has the form $\tau_{1} * \cdots * \tau_{i-1} * M t_{i} * \tau_{i+1} * \cdots * \tau_{k}$ and Pat has the form $\left(\right.$ Pat $_{1}, \ldots$, Pat $\left._{k}\right)$ and for all $1 \leq j \leq k$ such that $j \neq i$ we have $\operatorname{defaultPat}\left(\tau_{j}, C, d-1\right)=P a t_{j}$ and we have defaultPat $\left(M t_{i}, C, d-1\right)=\operatorname{Pat}_{i}$. Let $\tau_{i}=\hat{M} t_{i}$. Since $\tau^{\prime} \leq[\bar{\alpha} \mapsto \bar{\tau}]\left(\tau_{1} * \cdots * \tau_{k}\right)$, by Lemma 19 we have that $\tau^{\prime}$ has the form $\tau_{1}^{\prime} * \cdots * \tau_{k}^{\prime}$, where for all $1 \leq j \leq k$ we have $\tau_{j}^{\prime} \leq[\bar{\alpha} \mapsto \bar{\tau}] \tau_{j}$. Since $\vdash v: \tau^{\prime}$, by T-Tup we have that $v$ has the form $\left(v_{1}, \ldots, v_{k}\right)$ and for all $1 \leq j \leq k$ we have $\vdash v_{j}: \tau_{j}^{\prime}$. Therefore by Lemma 6 , for all $1 \leq j \leq k$ such that $j \neq i$ we have that there exists some $\rho_{j}$ such that $\operatorname{match}\left(v_{j}, P a t_{j}\right)=\rho_{j}$. We're given that owner $(M t, v)=C_{0}$, so by OwnerTupVAL we have owner $\left(M t_{i}, v_{i}\right)=$ $C_{0}$. Therefore by induction we have that there exists some $\rho_{i}$ such that $\operatorname{match}\left(v_{i}, P_{a t}\right)=\rho_{i}$. Then by E-MatchTup we have $\operatorname{match}(v, P a t)=\rho_{1} \cup \cdots \cup$ $\rho_{k}$.

Lemma 6. If $\vdash v: \tau^{\prime}$ and $\tau^{\prime} \leq \tau$ and $\tau=[\bar{\alpha} \mapsto \bar{\tau}] \tau_{0}$ and $\operatorname{defaultPat}\left(\tau_{0}\right.$, $\left.C_{0}, d\right)=$ Pat, then there exists $\rho$ such that match $(v$, Pat $)=\rho$.

Proof. By strong induction on the depth of the derivation of $\operatorname{defaultPat}\left(\tau_{0}, C_{0}, d\right)=$ Pat. Case analysis of the last rule in the derivation.

- Case DefZero or DefTypeVar or DefFunType. Then Pat has the form -, so by E-MatchWild we have $\operatorname{match}\left(v,{ }_{-}\right)=\{\}$.

- Case DefClassType. Then $\tau_{0}$ has the form $\left(\bar{\tau}_{0} C\right)$ and Pat has the form $(C\{\bar{V}=$ $\overline{P a t}\})$ and repType $\left(\overline{\tau_{0}} C\right)=\left\{\bar{V}: \overline{\tau^{\prime}}\right\}$ and defaultPat $\left(\overline{\tau^{\prime}}, C_{0}, d-1\right)=\overline{P a t}$ and $d>$ 0 . Since $\tau=[\bar{\alpha} \mapsto \bar{\tau}] \tau_{0}$, by Lemma 23 we have repType $(\tau)=[\bar{\alpha} \mapsto \bar{\tau}]\left\{\bar{V}: \overline{\tau^{\prime}}\right\}$. Further, $\tau=[\bar{\alpha} \mapsto \bar{\tau}]\left(\overline{\tau_{0}} C\right)=\left([\bar{\alpha} \mapsto \bar{\tau}] \overline{\tau_{0}} C\right)$. Since $\tau^{\prime} \leq \tau$, by Lemma $12 \tau^{\prime}$ has the form $\left(\overline{\tau_{1}} C^{\prime}\right)$. Since $\vdash v: \tau^{\prime}$, by T-REP $v$ has the form $\left(\overline{\tau_{1}} C^{\prime}\right)\left\{\overline{V_{1}}=\overline{v_{1}}\right\}$ and $\bullet \vdash\left(\overline{\tau_{1}} C^{\prime}\right)$ OK and repType $\left(\overline{\tau_{1}} C^{\prime}\right)=\left\{\overline{V_{1}}: \overline{\tau_{1}}\right\}$ and $\vdash \overline{v_{1}}: \overline{\tau_{1}^{\prime}}$ and $\overline{\tau_{1}^{\prime}} \leq \overline{\tau_{1}}$.

Since $\left(\overline{\tau_{1}} C^{\prime}\right) \leq\left([\bar{\alpha} \mapsto \bar{\tau}] \overline{\tau_{0}} C\right)$, by Lemma 15 we have $C^{\prime} \leq C$. Further, by Lemma 36 we have that $\left\{\overline{V_{1}}: \overline{\tau_{1}}\right\}=\left\{\bar{V}:[\bar{\alpha} \mapsto \bar{\tau}] \overline{\tau^{\prime}}, \overline{V_{2}}: \overline{\tau_{2}}\right\}$. Therefore there is some prefix $\overline{v_{3}}$ of $\overline{v_{1}}$ and some prefix $\overline{\tau_{3}}$ of $\overline{\tau_{1}^{\prime}}$ such that $\vdash \overline{v_{3}}: \overline{\tau_{3}}$ and $\overline{\tau_{3}} \leq[\bar{\alpha} \mapsto \bar{\tau}] \overline{\tau^{\prime}}$ and defaultPat $\left(\overline{\tau^{\prime}}, C_{0}, d-1\right)=\overline{\text { Pat }}$. Therefore by induction, $\operatorname{match}\left(\overline{v_{3}}, \overline{P a t}\right)=\bar{\rho}$. Therefore by E-MAtchClass we have $\operatorname{match}\left(\left(\overline{\tau_{1}} C^{\prime}\right)\left\{\overline{V_{1}}=\overline{v_{1}}\right\},(C\{\bar{V}=\overline{P a t}\})\right)=\bigcup \bar{\rho}$.

- Case DefTupType. Then $\tau_{0}$ has the form $\tau_{1} * \cdots * \tau_{k}$ and Pat has the form $\left(\right.$ Pat $_{1}, \ldots$, Pat $\left._{k}\right)$ and for all $1 \leq i \leq k$ we have defaultPat $\left(\tau_{i}, C_{0}, d-1\right)=$ Pat $_{i}$ and $d>0$. Since $\tau^{\prime} \leq[\bar{\alpha} \mapsto \bar{\tau}]\left(\tau_{1} * \cdots * \tau_{k}\right)$, by Lemma 19 we have that $\tau^{\prime}$ has the form $\tau_{1}^{\prime} * \cdots * \tau_{k}^{\prime}$, where for all $1 \leq i \leq k$ we have $\tau_{i}^{\prime} \leq[\bar{\alpha} \mapsto \bar{\tau}] \tau_{i}$. Since $\vdash v: \tau^{\prime}$, by T-Tup we have that $v$ has the form $\left(v_{1}, \ldots, v_{k}\right)$ and for all $1 \leq i \leq k$ we have $\vdash v_{i}: \tau_{i}^{\prime}$. Therefore by induction, for all $1 \leq i \leq k$ we have that there exists some $\rho_{i}$ such that $\operatorname{match}\left(v_{i}, P a t_{i}\right)=\rho_{i}$. Then by E-MatchTup we have $\operatorname{match}(v, P a t)=\rho_{1} \cup \cdots \cup \rho_{k}$. 
A.1.2 Ambiguity. The following lemma says that if a value has at least one applicable function case then it has a most-specific applicable case. The lemma thereby validates our modular ambiguity checking algorithm and requirement. The lemma is proven by induction on the number of applicable function cases that are not overridden by the given one. If there are none (other than the given case itself), then the given case is the most-specific applicable case. Otherwise, another applicable function case is not overridden by the given one. Then we show that, by modular ambiguity checking, there must exist a resolving case, and the result follows by induction on the resolving case.

Lemma 7 (Unambiguity). If $\vdash v: \tau$ and $S n \in \operatorname{dom}(\operatorname{Sig} T)$ and (extend fun $_{\mathrm{Mn}} \bar{\alpha} F$ Pat $) \in \operatorname{Sig} T(S n)$ and $\operatorname{match}(v, P a t)=\rho$, then there exists some $S n^{\prime} \in \operatorname{dom}(\operatorname{SigT})$, some (extend $\left.\mathrm{fun}_{M n} \overline{\alpha_{1}} F \mathrm{Pat}{ }^{\prime}\right) \in \operatorname{Sig} T\left(S n^{\prime}\right)$, and some $\rho^{\prime}$ such that $\operatorname{match}\left(v, P a t^{\prime}\right)=\rho^{\prime}$ and $\forall S^{\prime \prime} \in \operatorname{dom}(\operatorname{SigT}) . \forall\left(\right.$ extend fun $_{M n} \overline{\alpha_{2}} F$ Pat $\left.{ }^{\prime \prime}\right) \in$ $\operatorname{Sig} T\left(S n^{\prime \prime}\right) . \forall \rho^{\prime \prime} .\left(\left(\operatorname{match}\left(v, P a t^{\prime \prime}\right)=\rho^{\prime \prime} \wedge S n^{\prime} \cdot M n^{\prime} \neq S n^{\prime \prime} . M n^{\prime \prime}\right) \Rightarrow P a t^{\prime}<P a t^{\prime \prime}\right)$.

Proof. By (strong) induction on the number of function cases of $F$ that are applicable to $v$ but are not overridden by $S n . M n$. These are the cases of the form (extend fun $_{M n_{0}} \overline{\alpha_{0}} F P a t_{0}$ ) such that (extend fun $\left._{M n_{0}} \overline{\alpha_{0}} F P a t_{0}\right) \in \operatorname{Sig} T\left(S n_{0}\right)$ for some $S n_{0} \in \operatorname{dom}(\operatorname{SigT})$, and $\operatorname{match}\left(v, P a t_{0}\right)=\rho_{0}$ for some $\rho_{0}$, and Pat $\nless P a t_{0}$.

- Case there are zero function cases of $F$ that are applicable to $v$ but are not overridden by $S n . M n$. We're given that $S n \in \operatorname{dom}(\operatorname{SigT})$ and (extend $\operatorname{fun}_{M n} \bar{\alpha}$ $F P a t) \in \operatorname{SigT}(S n)$ and $\operatorname{match}(v, P a t)=\rho$. Further, since it cannot both be the case that Pat $\leq$ Pat and Pat $\not$ Pat, we have Pat $\nless$ Pat . Therefore, Sn.Mn itself is applicable to $v$ but is not overridden by $S n . M n$, so we have a contradiction.

- Case there is exactly one function case of $F$ that is applicable to $v$ but is not overridden by $S n . M n$. Then from the previous case we know that $S n . M n$ must itself be that function case. Therefore it follows that $\forall S n^{\prime \prime} \in \operatorname{dom}(\operatorname{Sig} T) . \forall\left(\right.$ extend $\left.\operatorname{fun}_{M n^{\prime \prime}} \overline{\alpha_{2}} F P a t^{\prime \prime}\right) \in \operatorname{Sig} T\left(S n^{\prime \prime}\right) . \forall \rho^{\prime \prime}$. $\left(\left(\operatorname{match}\left(v, P a t^{\prime \prime}\right)=\rho^{\prime \prime} \wedge S n \cdot M n \neq S n^{\prime \prime} \cdot M n^{\prime \prime}\right) \Rightarrow P a t<P a t^{\prime \prime}\right)$. Then the result follows.

- There are $k>1$ function cases of $F$ that are applicable to $v$ but are not overridden by Sn.Mn. Let (extend $\mathrm{fun}_{M n_{1}} \overline{\alpha_{3}} F \mathrm{Pat}_{1}$ ) be one such function case, so (extend $\left.\operatorname{fun}_{M n_{1}} \overline{\alpha_{3}} F P a t_{1}\right) \in \operatorname{Sig} T\left(S_{1} n_{1}\right)$ for some $S n_{1} \in \operatorname{dom}(\operatorname{SigT})$, and $\operatorname{match}\left(v, P a t_{1}\right)=\rho_{1}$ for some $\rho_{1}$, and Pat $\nless P a t_{1}$. Since $k>1$, at least one of the function cases satisfying the conditions is not $S n . M n$, so assume without loss of generality that $S n \cdot M n \neq S n_{1} \cdot M n_{1}$.

By CASEOK we have matchType $\left(\tau_{0}, P a t\right)=\left(\Gamma_{0}, \tau_{0}^{\prime}\right)$ and matchType $\left(\tau_{1}\right.$, $\left.P a t_{1}\right)=\left(\Gamma_{1}, \tau_{1}^{\prime}\right)$. We're given that $\vdash v: \tau$. Finally, we saw above that $\operatorname{match}(v, P a t)=\rho$ and $\operatorname{match}\left(v\right.$, Pat $\left._{1}\right)=\rho_{1}$. Therefore by Lemma 30 there exists some Pat int such that Pat $\cap P a t_{1}=P a t_{\text {int }}$. Further, by Lemma 8 we have $\operatorname{dom}(\operatorname{Sig} T) \vdash\left(P a t, P a t_{1}\right)$ unambiguous. Therefore by PAIRAMB we have that $P a t \not P a t_{1}$ and there exists some $S n_{2} \in \operatorname{dom}(\operatorname{Sig} T)$ and some (extend fun $\left._{M n_{2}} \overline{\alpha_{4}} F P a t_{2}\right) \in \operatorname{Sig} T\left(S_{2}\right)$ such that $P a t_{i n t} \cong P a t_{2}$. Since match $(v, P a t)=$ $\rho$ and $\operatorname{match}\left(v, P_{a t}\right)=\rho_{1}$ and Pat $\cap P a t_{1}=P_{a t} t_{i n t}$, by Lemma 31 there exists some $\rho_{\text {int }}$ such that $\operatorname{match}\left(v, P a t_{i n t}\right)=\rho_{\text {int }}$. Then since Pat $t_{i n t} \leq P_{\text {Pat }}$, by Lemma 25 there exists $\rho_{2} \operatorname{such}$ that $\operatorname{match}\left(v, \operatorname{Pat}_{2}\right)=\rho_{2}$. 
So we have shown there exists some $S n_{2} \in \operatorname{dom}(\operatorname{Sig} T)$ and some (extend fun $\left._{\mathrm{Mn}_{2}} \overline{\alpha_{4}} F P a t_{2}\right) \in \operatorname{Sig} T\left(S n_{2}\right)$ and some $\rho_{2} \operatorname{such}$ that $\operatorname{match}\left(v, P a t_{2}\right)=\rho_{2}$. Let $l$ be the number of function cases of $F$ that are applicable to $v$ but are not overridden by $S n_{2} \cdot M n_{2}$. This case is finished by showing that $l<k$, so that the result follows by induction with respect to $S n_{2} \cdot M n_{2}$.

First we show that $l \leq k$. Consider some $S n_{0} \in \operatorname{dom}(\operatorname{SigT})$ ), some (extend fun $\left._{M n_{0}} \overline{\alpha_{0}} F P a t_{0}\right) \in \operatorname{Sig} T\left(S n_{0}\right)$, and some $\rho_{0} \operatorname{such}$ that match $\left(v, P a t_{0}\right)=\rho_{0}$ and $\mathrm{Pat}_{2} \nless \mathrm{Pat}_{0}$. We will show that also Pat $\nless P a t_{0}$.

Since Pat $\cap$ Pat $_{1}=$ Pat $_{\text {int }}$, by Lemma 28 we have that Pat ${ }_{\text {int }} \leq P a t$ and Pat $_{\text {int }} \leq$ Pat $_{1}$. Since Pat $t_{2} \leq$ Pat $_{\text {int }}$, by Lemma 26 also Pat $2 \leq$ Pat and Pat $t_{2} \leq$ $P a t_{1}$. Since $\mathrm{Pat}_{2} \nless P a t_{0}$, either Pat $t_{2} \nless \mathrm{Pat}_{0}$ or $\mathrm{Pat}_{0} \leq \mathrm{Pat}_{2}$. We consider these cases in turn.

- Case Pat $t_{2} \not \leq P a t_{0}$. Suppose Pat $\leq P a t_{0}$. Since Pat $t_{2} \leq$ Pat, by Lemma 26 we have $\mathrm{Pat}_{2} \leq \mathrm{Pat}_{0}$, contradicting the assumption of this case. Therefore Pat $\not$ Pat $_{0}$, so also Pat $\nless P a t_{0}$.

- Case Pat $t_{0} \leq$ Pat $_{2}$. We showed above that Pat $2 \leq$ Pat. Then by Lemma 26

Pat $_{0} \leq$ Pat, so Pat $\nless$ Pat ${ }_{0}$.

Therefore we have shown that every function case of $F$ that is applicable to $v$ and is not overridden by $S n_{2} \cdot M n_{2}$ is also not overridden by $S n . M n$, so $l \leq k$. To finish the proof, we show that there exists a function case of $F$ that is applicable to $v$ and is not overridden by $S n . M n$ but is overridden by $S n_{2} \cdot M n_{2}$. We showed in the first case above that $S n . M n$ is not overridden by itself. We will show that $S n . M n$ is overridden by $S n_{2} \cdot M n_{2}$. We do this by proving that $\mathrm{Pat}_{2}<$ Pat. We showed above that Pat $t_{2} \leq$ Pat, so we simply need to prove that $P a t \nless P a t_{2}$. Suppose Pat $\leq P a t_{2}$. We're given that Pat $\nless P a t_{1}$ and $P a t ¥ P a t_{1}$. Therefore, Pat $\not P a t_{1}$. On the other hand, since Pat $\leq P_{a t}$ and Pat $t_{2} \leq P a t_{1}$, by Lemma 26 we have Pat $\leq P a t_{1}$, and we have a contradiction.

This lemma says that every pair of function cases belonging to the same function is unambiguous. If the two cases are both available during some structure's modular ITC, then the result follows from that structure's ambiguity checks. Otherwise, the modular ambiguity requirement ensures that the cases are disjoint, so they are also unambiguous.

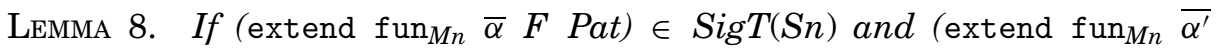

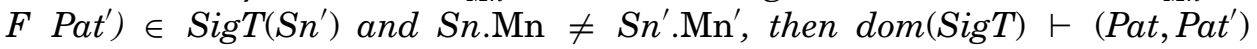
unambiguous.

Proof. Since (extend $\mathrm{fun}_{M n} \bar{\alpha} F$ Pat) $\in \operatorname{SigT}(\mathrm{Sn})$, by StructOK we have $\overline{S n} \subseteq \operatorname{dom}(\operatorname{Sig} T)$ and $\overline{S n} \vdash$ (extend fun $_{M n} \bar{\alpha} F$ Pat $=E$ ) OK in $S n$ for some $\overline{S n}$ and $E$, so by CASEOK we have $S n ; \overline{S n} \vdash$ extend fun $_{M n} \bar{\alpha} F$ Pat unambiguous. Similarly, $\overline{S n^{\prime}} \subseteq \operatorname{dom}(S i g T)$ and $S n^{\prime} ; \overline{S n^{\prime}} \vdash$ extend fun $_{M n^{\prime}} \overline{\alpha^{\prime}} F$ Pat' unambiguous, for some $\overline{S n^{\prime}}$. There are several cases.

- Case $S n^{\prime} \in \overline{S n}$. Since $S n \cdot M n \neq S n^{\prime} \cdot M n^{\prime}$ and $S n ; \overline{S n} \vdash$ extend $\operatorname{fun}_{M n} \bar{\alpha} F$ Pat unambiguous, by AmB we have $\overline{S n} \vdash\left(\right.$ pat, pat $\left.t^{\prime}\right)$ unambiguous. Since $\overline{S n} \subseteq$ $\operatorname{dom}(\operatorname{SigT})$, by AмB also $\operatorname{dom}(\operatorname{Sig} T) \vdash\left(\right.$ Pat, $\left.P a t^{\prime}\right)$ unambiguous.

- Case $S n \in \overline{S n}$. Symmetric to the above case. 
- Case $S n^{\prime} \notin \overline{S n}$ and $S n \notin \overline{S n^{\prime}}$. Since $S n ; \overline{S n} \vdash$ extend fun fun $_{M n} \bar{\alpha}$ Pat unambiguous, by Amb we have $F=S n_{1} . F n$ and (fun $\left.\overline{\alpha_{1}} F n: M t \rightarrow \tau\right) \in S i g T\left(S n_{1}\right)$ and $S n=S n_{1} \vee$ owner $(M t, P a t)=S n . C n$. Similarly, $S n^{\prime}=S n_{1} \vee\left(\right.$ owner $\left(M t, P a t^{\prime}\right)=$ $S n^{\prime} . \mathrm{Cn}^{\prime}$. We have three sub-cases.

-Case $S n^{\prime}=S n_{1}$. Since $\overline{S n} \vdash$ (extend fun $_{M n} \bar{\alpha} F P a t=E$ ) OK in $S n$, by CASEOK we have $\overline{S n} \vdash S n^{\prime}$.Fn ITCUses. Then by FunITCUses $S n^{\prime} \in \overline{S n}$, so we have a contradiction.

- Case $S n=S n_{1}$. Symmetric to the above case.

- Case owner $(M t, P a t)=S n . C n$ and owner $\left(M t, P a t^{\prime}\right)=S n^{\prime} . C n^{\prime}$. First we show that Pat and Pat' are disjoint: there does not exist Pat $t_{0}$ such that Pat $\cap$ $P a t^{\prime}=P^{\prime} t_{0}$. Suppose not. Then by Lemma 29 either $\mathrm{Sn} . \mathrm{Cn} \leq \mathrm{Sn}^{\prime} . \mathrm{Cn}^{\prime}$ or $S n^{\prime} . C n^{\prime} \leq S n . C n$. There are two subcases.

- Case $S n . C n \leq S n^{\prime} . C n^{\prime}$. By StRuctOK $\overline{S n} \vdash\left(<\right.$ abstract $>$ class $\left.\overline{\alpha_{4}} C n \ldots\right)$ $\mathrm{OK}$ in $S n$, so by ClassOK $\overline{S n} \vdash S n . C n$ ITCTransUses. Since $S n . C n \leq$ $S n^{\prime} . C n^{\prime}$, by Lemma 37 we have $S n^{\prime} \in \overline{S n}$, which is a contradiction.

- Case $S n^{\prime} . C n^{\prime} \leq S n$.Cn. Symmetric to the above case.

So we have shown that there does not exist Pat $t_{0}$ such that Pat $\cap$ Pat $^{\prime}=$ Pat $_{0}$. Then by the contrapositive of Lemma 27, Pat $\Varangle P a t^{\prime}$, so also Pat $¥$ Pat'. Then by PaIRAmb we have $\operatorname{dom}(\operatorname{Sig} T) \vdash\left(P a t, P a t^{\prime}\right)$ unambiguous.

\section{A.2 Type Preservation}

The type preservation theorem is straightforward except for the case when $E$ is a function application. That case follows easily from Lemmas 9 and 10.

Theorem 2 (Type Preservation). If $\vdash E: \tau$ and $E \longrightarrow E^{\prime}$ then $\vdash E^{\prime}: \tau^{\prime}$, for some $\tau^{\prime}$ such that $\tau^{\prime} \leq \tau$.

Proof. By (strong) induction on the depth of the derivation of $E \longrightarrow E^{\prime}$. Case analysis of the last rule used in the derivation.

- Case E-New. Then $E$ has the form $C t(\bar{E})$ and $E^{\prime}$ has the form $C t\left\{\overline{V_{0}}=\overline{E_{0}}\right\}$ and $C t=(\bar{\tau} C)$ and concrete $(C)$ and $\operatorname{rep}(C t(\bar{E}))=\left\{\overline{V_{0}}=\overline{E_{0}}\right\}$. Since $\vdash E$ : $\tau$, by T-New we have $\tau=C t$ and \{\}$; \bullet \vdash C t(\bar{E})$ OK. Then by T-Constr we have $\bullet \vdash C t$ OK. Therefore by Lemmas 33 and 34 there exists $\overline{\tau_{0}}$ such that repType $(C t)=\left\{\overline{V_{0}}: \overline{\tau_{0}}\right\}$, and by Lemma 35 we have $\vdash \overline{E_{0}}: \overline{\tau_{0}^{\prime}}$, where $\overline{\tau_{0}^{\prime}} \leq \overline{\tau_{0}}$. Then by T-Rep we have $\vdash C t\left\{\overline{V_{0}}=\overline{E_{0}}\right\}: C t$, and by SubTRef we have $C t \leq C t$.

- Case E-REP. Then $E$ has the form $C t\left\{\overline{V_{0}}=\overline{v_{0}}, V_{0}=E_{0}, \overline{V_{1}}=\overline{E_{1}}\right\}$ and $E^{\prime}$ has the form $C t\left\{\overline{V_{0}}=\overline{v_{0}}, V_{0}=E_{0}^{\prime}, \overline{V_{1}}=\overline{E_{1}}\right\}$ and $E_{0} \longrightarrow E_{0}^{\prime}$. Since $\vdash E$ : $\tau$, by T-ReP we have $\tau=C t=(\bar{\tau} C)$ and concrete $(C)$ and $\bullet \vdash C t$ OK and repType $(C t)=\left\{\overline{V_{0}}: \overline{\tau_{0}}, V_{0}: \tau_{0}, \overline{V_{1}}: \overline{\tau_{1}}\right\}$ and $\vdash \overline{v_{0}}: \overline{\tau_{0}^{\prime}}$ and $\overline{\tau_{0}^{\prime}} \leq \overline{\tau_{0}}$ and $\vdash E_{0}: \tau_{0}^{\prime}$ and $\tau_{0}^{\prime} \leq \tau_{0}$ and $\vdash \overline{E_{1}}: \overline{\tau_{1}^{\prime}}$ and $\overline{\tau_{1}^{\prime}} \leq \overline{\tau_{1}}$. By induction we have $\vdash E_{0}^{\prime}: \tau_{0}^{\prime \prime}$, for some $\tau_{0}^{\prime \prime}$ such that $\tau_{0}^{\prime \prime} \leq \tau_{0}^{\prime}$. Therefore by SubTTrans $\tau_{0}^{\prime \prime} \leq \tau_{0}$. Then by T-REP $\vdash C t\left\{\overline{V_{0}}=\overline{v_{0}}, V_{0}=E_{0}^{\prime}, \overline{V_{1}}=\overline{E_{1}}\right\}: C t$, and by SubTReF $C t \leq C t$.

- Case E-Tup. Then $E$ has the form $\left(v_{1}, \ldots, v_{i-1}, E_{i}, \ldots, E_{k}\right)$ and $E^{\prime}$ has the form $\left(v_{1}, \ldots, v_{i-1}, E_{i}^{\prime}, E_{i+1}, \ldots, E_{k}\right)$ and $E_{i} \longrightarrow E_{i}^{\prime}$, where $1 \leq i \leq k$. Since $\vdash E: \tau$, by T-Tup $\tau$ has the form $\tau_{1} * \cdots * \tau_{k}$ and $\vdash v_{j}: \tau_{j}$ for all $1 \leq j<i$ 
and $\vdash E_{j}: \tau_{j}$ for all $i \leq j \leq k$. By induction we have $\vdash E_{i}^{\prime}: \tau_{i}^{\prime}$ for some $\tau_{i}^{\prime}$ such that $\tau_{i}^{\prime} \leq \tau_{i}$. Then by T-Tup we have $\vdash\left(v_{1}, \ldots, v_{i-1}, E_{i}^{\prime}, E_{i+1}, \ldots, E_{k}\right)$ : $\tau_{1} * \cdots * \tau_{i-1} * \tau_{i}^{\prime} * \tau_{i+1} * \cdots * \tau_{k}$. By SUBTREF we have $\tau_{j} \leq \tau_{j}$ for all $1 \leq j \leq k$, so by SuBTTup we have $\tau_{1} * \cdots * \tau_{i-1} * \tau_{i}^{\prime} * \tau_{i+1} * \cdots * \tau_{k} \leq \tau_{1} * \cdots * \tau_{k}$.

- Case E-App1. Then $E$ has the form $E_{1} E_{2}$ and $E^{\prime}$ has the form $E_{1}^{\prime} E_{2}$ and $E_{1} \longrightarrow E_{1}^{\prime}$. Since $\vdash E: \tau$, by (T-App) we have $\vdash E_{1}: \tau_{2} \rightarrow \tau$ and $\vdash E_{2}: \tau_{2}^{\prime}$ and $\tau_{2}^{\prime} \leq \tau_{2}$. By induction $\vdash E_{1}^{\prime}: \tau^{\prime}$, for some $\tau^{\prime}$ such that $\tau^{\prime} \leq \tau_{2} \rightarrow \tau$. By Lemma $17 \tau^{\prime}$ has the form $\tau_{2}^{\prime \prime} \rightarrow \tau^{\prime \prime}$, where $\tau_{2} \leq \tau_{2}^{\prime \prime}$ and $\tau^{\prime \prime} \leq \tau$. Therefore by SubTTRans we have $\tau_{2}^{\prime} \leq \tau_{2}^{\prime \prime}$, so by T-ApP we have $\vdash E_{1}^{\prime} E_{2}: \tau^{\prime \prime}$, where $\tau^{\prime \prime} \leq \tau$.

- Case E-App2. Then $E$ has the form $v_{1} E_{2}$ and $E^{\prime}$ has the form $v_{1} E_{2}^{\prime}$ and $E_{2} \longrightarrow$ $E_{2}^{\prime}$. Since $\vdash E: \tau$, by T-App we have $\vdash v_{1}: \tau_{2} \rightarrow \tau$ and $\vdash E_{2}: \tau_{2}^{\prime}$ and $\tau_{2}^{\prime} \leq \tau_{2}$. By induction $\vdash E_{2}^{\prime}: \tau_{2}^{\prime \prime}$, for some $\tau_{2}^{\prime \prime}$ such that $\tau_{2}^{\prime \prime} \leq \tau_{2}^{\prime}$. By SuBTTrans we have $\tau_{2}^{\prime \prime} \leq \tau_{2}$, so by T-ApP we have $\vdash v_{1} E_{2}^{\prime}: \tau$ and by SuBTREF we have $\tau \leq \tau$.

- Case E-AppRed. Then $E=(\bar{\tau} F) v$ and $E^{\prime}=\left[\overline{I_{0}} \mapsto \overline{v_{0}}\right] E_{0}$ and most-specificcase-for $((\bar{\tau} F), v)=\left(\left\{\left(\overline{I_{0}}, \overline{v_{0}}\right)\right\}, E_{0}\right)$. Since $\vdash E: \tau$, by T-App we have $\vdash(\bar{\tau} F)$ : $\tau_{2} \rightarrow \tau$ and $\vdash v: \tau_{2}^{\prime}$ and $\tau_{2}^{\prime} \leq \tau_{2}$. Then by T-Fun we have and $F=S n . F n$ and $\tau_{2} \rightarrow \tau=[\bar{\alpha} \mapsto \bar{\tau}]\left(\hat{M} t \rightarrow \tau_{0}\right)$ and (fun $\left.\bar{\alpha} F n: M t \rightarrow \tau_{0}\right) \in \operatorname{SigT}(S n)$ and - $\vdash \bar{\tau}$ OK. By Lookup we have $E_{0}=\left[\overline{\alpha_{0}} \mapsto \bar{\tau}\right] E_{0}^{\prime}$ and (extend fun Mn $_{\overline{\alpha_{0}}} F$ $\left.P a t=E_{0}^{\prime}\right) \in S T\left(S n^{\prime}\right)$ and $\operatorname{match}(v, P a t)=\left\{\left(\overline{I_{0}}, \overline{v_{0}}\right)\right\}$. Then by CASEOK we have matchType $\left(\left[\bar{\alpha} \mapsto \overline{\alpha_{0}}\right] \hat{M} t, P a t\right)=\left(\Gamma, \tau^{\prime \prime}\right)$ and $\Gamma ; \overline{\alpha_{0}} \vdash E_{0}^{\prime}: \tau_{0}^{\prime}$ and $\tau_{0}^{\prime} \leq\left[\bar{\alpha} \mapsto \overline{\alpha_{0}}\right] \tau_{0}$.

By Lemma 24 we have matchType $\left(\left[\overline{\alpha_{0}} \mapsto \bar{\tau}\right]\left[\bar{\alpha} \mapsto \overline{\alpha_{0}}\right] \hat{M} t\right.$, Pat $)=\left(\left[\overline{\alpha_{0}} \mapsto\right.\right.$ $\left.\bar{\tau}] \Gamma,\left[\overline{\alpha_{0}} \mapsto \bar{\tau}\right] \tau^{\prime \prime}\right)$. By FunOK we have $\bar{\alpha} \vdash \hat{M} t$ OK, so by Lemma 11 all type variables in $\hat{M} t$ are in $\bar{\alpha}$. Therefore $\left[\overline{\alpha_{0}} \mapsto \bar{\tau}\right]\left[\bar{\alpha} \mapsto \overline{\alpha_{0}}\right] \hat{M} t$ is equivalent to $[\bar{\alpha} \mapsto$ $\bar{\tau}] \hat{M} t=\tau_{2}$, so we have matchType $\left(\tau_{2}\right.$, Pat $)=\left(\left[\overline{\alpha_{0}} \mapsto \bar{\tau}\right] \Gamma,\left[\overline{\alpha_{0}} \mapsto \bar{\tau}\right] \tau^{\prime \prime}\right)$. Then by Lemma 10 we have $\tau_{2}^{\prime} \leq\left[\overline{\alpha_{0}} \mapsto \bar{\tau}\right] \tau^{\prime \prime}$ and $\operatorname{dom}\left(\left[\overline{\alpha_{0}} \mapsto \bar{\tau}\right] \Gamma\right)=\operatorname{dom}\left(\left\{\left(\overline{I_{0}}, \overline{v_{0}}\right)\right\}\right)$ and for each $\left(I_{x}, \tau_{x}\right) \in\left[\overline{\alpha_{0}} \mapsto \bar{\tau}\right] \Gamma$, there exists $\left(I_{x}, v_{x}\right) \in\left\{\left(\overline{I_{0}}, \overline{v_{0}}\right)\right\}$ such that $\vdash v_{x}: \tau_{x}^{\prime}$, where $\tau_{x}^{\prime} \leq \tau_{x}$.

By Lemma 22 we have $\left[\overline{\alpha_{0}} \mapsto \bar{\tau}\right] \Gamma ; \bullet \vdash\left[\overline{\alpha_{0}} \mapsto \bar{\tau}\right] E_{0}^{\prime}:\left[\overline{\alpha_{0}} \mapsto \bar{\tau}\right] \tau_{0}^{\prime}$. Then by Lemma $9 \vdash\left[\overline{I_{0}} \mapsto \overline{v_{0}}\right]\left[\overline{\alpha_{0}} \mapsto \bar{\tau}\right] E_{0}^{\prime}: \tau_{s u b}$ and $\tau_{\text {sub }} \leq\left[\overline{\alpha_{0}} \mapsto \bar{\tau}\right] \tau_{0}^{\prime}$. By Lemma 21 we have $\left[\overline{\alpha_{0}} \mapsto \bar{\tau}\right] \tau_{0}^{\prime} \leq\left[\overline{\alpha_{0}} \mapsto \bar{\tau}\right]\left[\bar{\alpha} \mapsto \overline{\alpha_{0}}\right] \tau_{0}$. By FunOK we have $\bar{\alpha} \vdash \tau_{0}$ OK, so by Lemma 11 all type variables in $\tau_{0}$ are in $\bar{\alpha}$. Therefore $\left[\overline{\alpha_{0}} \mapsto \bar{\tau}\right]\left[\bar{\alpha} \mapsto \overline{\alpha_{0}}\right] \tau_{0}$ is equivalent to $[\bar{\alpha} \mapsto \bar{\tau}] \tau_{0}=\tau$, so we have $\left[\overline{\alpha_{0}} \mapsto \bar{\tau}\right] \tau_{0}^{\prime} \leq \tau$. Then by SubTTRANS we have $\tau_{\text {sub }} \leq \tau$. Therefore we have shown $\vdash E^{\prime}: \tau_{\text {sub }}$ and $\tau_{\text {sub }} \leq \tau$.

This is a standard lemma showing that type-correct substitution preserves the well-typedness of an expression.

Lemma 9 (Substitution). If $\Gamma ; \overline{\alpha_{0}} \vdash E: \tau$ and $\Gamma=\left\{\left(\overline{I_{0}}, \overline{\tau_{0}}\right)\right\}$ and $\Gamma_{0} ; \overline{\alpha_{0}} \vdash \overline{E_{0}}$ : $\overline{\tau_{0}}$ and $\overline{\tau_{0}^{\prime}} \leq \overline{\tau_{0}}$, then $\Gamma_{0} ; \overline{\alpha_{0}} \vdash\left[\overline{I_{0}} \mapsto \overline{E_{0}}\right] E: \tau^{\prime}$, for some $\tau^{\prime}$ such that $\tau^{\prime} \leq \tau$.

Proof. By (strong) induction on the depth of the derivation of $\Gamma ; \overline{\alpha_{0}} \vdash E: \tau$. Case analysis of the last rule used in the derivation.

- Case T-ID. Then $E=I$ and $(I, \tau) \in \Gamma$, so $I=I_{j}$ and $\tau=\tau_{j}$ for some $1 \leq j \leq k$, where $\overline{I_{0}}=I_{1}, \ldots, I_{k}$ and $\overline{\tau_{0}}=\tau_{1}, \ldots, \tau_{k}$ and $\overline{E_{0}}=E_{1}, \ldots, E_{k}$. Therefore $\left[\overline{I_{0}} \mapsto \overline{E_{0}}\right] E=E_{j}$. Since we're given that $\Gamma_{0} ; \overline{\alpha_{0}} \vdash E_{j}: \tau_{j}^{\prime}$ and $\tau_{j}^{\prime} \leq \tau_{j}$, the result is shown. 
- Case T-New. Then $E=C t(\bar{E})$ and $\tau=C t$ and $\Gamma ; \overline{\alpha_{0}} \vdash C t(\bar{E})$ OK and $C t=$ $\left(\overline{\tau_{1}}\right.$ Sn.Cn $)$ and concrete(Sn.Cn). Then by T-Constr we have $\overline{\alpha_{0}} \vdash C t$ OK and (<abstract > class $\left.\overline{\alpha_{1}} \operatorname{Cn}(\bar{I}: \bar{\tau}) \ldots\right) \in \operatorname{SigT}(S n)$ and $\Gamma ; \overline{\alpha_{0}} \vdash \bar{E}: \overline{\tau^{\prime}}$ and $\overline{\tau^{\prime}} \leq\left[\overline{\alpha_{1}} \mapsto \overline{\tau_{1}}\right] \bar{\tau}$. Since $\left[\overline{I_{0}} \mapsto \overline{E_{0}}\right] C t=C t$ and $\left[\overline{I_{0}} \mapsto \overline{E_{0}}\right]$ Sn.Cn $=$ Sn.Cn, we have $\overline{\alpha_{0}} \vdash\left[\overline{I_{0}} \mapsto \overline{E_{0}}\right] C t$ OK and concrete $\left(\left[\overline{I_{0}} \mapsto \overline{E_{0}}\right] S n . C n\right)$. By induction we have $\Gamma_{0} ; \overline{\alpha_{0}} \vdash\left[\overline{I_{0}} \mapsto \overline{E_{0}}\right] \bar{E}: \overline{\tau^{\prime \prime}}$ and $\overline{\tau^{\prime \prime}} \leq \overline{\tau^{\prime}}$. Then by SubTTRans we have $\overline{\tau^{\prime \prime}} \leq\left[\overline{\alpha_{1}} \mapsto \overline{\tau_{1}}\right] \bar{\tau}$. Therefore by T-ConstR we have $\Gamma_{0} ; \overline{\alpha_{0}} \vdash\left[\overline{I_{0}} \mapsto \overline{E_{0}}\right] E$ OK, so by T-New we have $\Gamma_{0} ; \overline{\alpha_{0}} \vdash\left[\overline{I_{0}} \mapsto \overline{E_{0}}\right] E: \tau$. By SubTREF we have $\tau \leq \tau$, so the result is shown.

- Case T-Rep. Then $E=C t\{\bar{V}=\bar{E}\}$ and $\tau=C t$ and $\overline{\alpha_{0}} \vdash C t$ OK and $C t=$ $\left(\overline{\tau_{1}}\right.$ Sn.Cn) and concrete $(S n . C n)$ and repType $(C t)=\{\bar{V}: \bar{\tau}\}$ and $\Gamma ; \overline{\alpha_{0}} \vdash \bar{E}: \overline{\tau^{\prime}}$ and $\overline{\tau^{\prime}} \leq \bar{\tau}$. Since $\left[\overline{I_{0}} \mapsto \overline{E_{0}}\right] C t=C t$ and $\left[\overline{I_{0}} \mapsto \overline{E_{0}}\right] S n . C n=S n . C n$, we have $\overline{\alpha_{0}} \vdash\left[\overline{I_{0}} \mapsto \overline{E_{0}}\right] C t$ OK and concrete $\left(\left[\overline{I_{0}} \mapsto \overline{E_{0}}\right] S n . C n\right)$ and repType $\left(\left[\overline{I_{0}} \mapsto\right.\right.$ $\left.\left.\overline{E_{0}}\right] C t\right)=\{\bar{V}: \bar{\tau}\}$. By induction we have $\Gamma_{0} ; \overline{\alpha_{0}} \vdash\left[\overline{I_{0}} \mapsto \overline{E_{0}}\right] \bar{E}: \overline{\tau^{\prime \prime}}$ and $\overline{\tau^{\prime \prime}} \leq \overline{\tau^{\prime}}$. Then by SubTTRans we have $\overline{\tau^{\prime \prime}} \leq \bar{\tau}$, so by T-Rep we have $\Gamma_{0} ; \overline{\alpha_{0}} \vdash\left[\overline{I_{0}} \mapsto\right.$ $\left.\overline{E_{0}}\right] E: \tau$. By SubTREF we have $\tau \leq \tau$, so the result is shown.

- Case T-Fun. Then since $\Gamma$ is not used in T-Fun and $\Gamma ; \overline{\alpha_{0}} \vdash E: \tau$, also $\Gamma_{0} ; \overline{\alpha_{0}} \vdash$ $E: \tau$. Further, we have $E=F v$, so $\left[\overline{I_{0}} \mapsto \overline{E_{0}}\right] E=E$. Therefore $\Gamma_{0} ; \overline{\alpha_{0}} \vdash\left[\overline{I_{0}} \mapsto\right.$ $\left.\overline{E_{0}}\right] E: \tau$, and by SuBTREF $\tau \leq \tau$, so the result is shown.

- Case T-Tup. Then $E=\left(E_{1}, \ldots, E_{k}\right)$ and $\tau=\tau_{1} * \cdots * \tau_{k}$ and for all $1 \leq j \leq k$ we have $\Gamma ; \overline{\alpha_{0}} \vdash E_{j}: \tau_{j}$. Then by induction, for all $1 \leq j \leq k$ we have $\Gamma_{0} ; \overline{\alpha_{0}} \vdash\left[\overline{I_{0}} \mapsto \overline{E_{0}}\right] E_{j}: \tau_{j}^{\prime}$ and $\tau_{j}^{\prime} \leq \tau_{j}$. Then by T-Tup we have $\Gamma_{0} ; \overline{\alpha_{0}} \vdash\left[\overline{I_{0}} \mapsto\right.$ $\left.\overline{E_{0}}\right]\left(E_{1}, \ldots, E_{k}\right): \tau_{1}^{\prime} * \cdots * \tau_{k}^{\prime}$. Finally, by SuBTTup we have $\tau_{1}^{\prime} * \cdots * \tau_{k}^{\prime} \leq \tau_{1} * \cdots * \tau_{k}$.

- Case T-Apr. Then $E=E_{1} E_{2}$ and $\Gamma ; \overline{\alpha_{0}} \vdash E_{1}: \tau_{2} \rightarrow \tau$ and $\Gamma ; \overline{\alpha_{0}} \vdash E_{2}: \tau_{2}^{\prime}$ and $\tau_{2}^{\prime} \leq \tau_{2}$. By induction we have $\Gamma_{0} ; \overline{\alpha_{0}} \vdash\left[\overline{I_{0}} \mapsto \overline{E_{0}}\right] E_{1}: \tau_{0}$ and $\tau_{0} \leq \tau_{2} \rightarrow \tau$. Also by induction we have $\Gamma_{0} ; \overline{\alpha_{0}} \vdash\left[\overline{I_{0}} \mapsto \overline{E_{0}}\right] E_{2}: \tau_{2}^{\prime \prime}$ and $\tau_{2}^{\prime \prime} \leq \tau_{2}^{\prime}$. Then by SubTTRans we have $\tau_{2}^{\prime \prime} \leq \tau_{2}$. By Lemma $17 \tau_{0}$ has the form $\tau_{\text {arg }} \rightarrow \tau_{\text {res }}$, where $\tau_{2} \leq \tau_{\text {arg }}$ and $\tau_{\text {res }} \leq \tau$. Therefore by SuBTTRans we have $\tau_{2}^{\prime \prime} \leq \tau_{\text {arg }}$. Therefore by T-ApP we have $\Gamma_{0} ; \overline{\alpha_{0}} \vdash\left[\overline{I_{0}} \mapsto \overline{E_{0}}\right]\left(E_{1}^{\prime} E_{2}^{\prime}\right): \tau_{\text {res }}$. We saw above that $\tau_{\text {res }} \leq \tau$, so the result is shown.

This lemma relates the results of pattern matching to its static approximation. In particular, the type of any value matching a pattern is a subtype of the pattern's type, and similarly for values bound to identifiers in the pattern. The proof is straightforward.

Lemma 10. If $\vdash v: \tau^{\prime \prime}$ and $\tau^{\prime \prime} \leq \tau$ and $\operatorname{match}(v$, Pat $)=\rho$ and matchType $(\tau$, Pat $)=\left(\Gamma, \tau^{\prime}\right)$, then (1) $\tau^{\prime \prime} \leq \tau^{\prime}$; and $(2) \operatorname{dom}(\Gamma)=\operatorname{dom}(\rho)$ and (3) for each $\left(I_{0}, \tau_{0}\right) \in \Gamma$, there exists $\left(I_{0}, v_{0}\right) \in \rho$ such that $\vdash v_{0}: \tau_{0}^{\prime}$, for some $\tau_{0}^{\prime}$ such that $\tau_{0}^{\prime} \leq \tau_{0}$.

PRoof. By (strong) induction on the length of the derivation of match $(v, P a t)=\rho$. Case analysis of the last rule used in the derivation:

- Case E-MatchWild. Then Pat has the form - and $\rho=\{\}$. By T-MatchWild we have $\Gamma=\{\}$ and $\tau^{\prime}=\tau$. Therefore, conditions 1 and 2 are shown, and condition 3 holds vacuously. 
- Case E-MatchBind. Then Pat has the form $I$ as Pat and $\rho=\rho^{\prime} \cup\{(I, v)\}$ and $\operatorname{match}\left(v, P a t^{\prime}\right)=\rho^{\prime}$. By T-MatchBind we have $\Gamma=\Gamma^{\prime} \cup\left\{\left(I, \tau^{\prime}\right)\right\}$ and matchType $\left(\tau, P a t^{\prime}\right)=\left(\Gamma^{\prime}, \tau^{\prime}\right)$. By induction we have $\tau^{\prime \prime} \leq \tau^{\prime}$ and $\operatorname{dom}\left(\Gamma^{\prime}\right)=$ $\operatorname{dom}\left(\rho^{\prime}\right)$ and for each $\left(I_{0}, \tau_{0}\right) \in \Gamma^{\prime}$, there exists $\left(I_{0}, v_{0}\right) \in \rho^{\prime}$ such that $\vdash v_{0}: \tau_{0}^{\prime}$, where $\tau_{0}^{\prime} \leq \tau_{0}$. Therefore, we have $\tau^{\prime \prime} \leq \tau^{\prime}$ and $\operatorname{dom}\left(\Gamma^{\prime} \cup\left\{\left(I, \tau^{\prime}\right)\right\}\right)=\operatorname{dom}\left(\rho^{\prime} \cup\right.$ $\{(I, v)\})$ and for each $\left(I_{0}, \tau_{0}\right) \in \Gamma^{\prime} \cup\left\{\left(I, \tau^{\prime}\right)\right\}$, there exists $\left(I_{0}, v_{0}\right) \in \rho^{\prime} \cup\{(I, v)\}$ such that $\vdash v_{0}: \tau_{0}^{\prime}$, where $\tau_{0}^{\prime} \leq \tau_{0}$.

- Case E-MatchTup. Then $v=\left(v_{1}, \ldots, v_{k}\right)$ and Pat has the form $\left(\right.$ Pat $_{1}, \ldots$, Pat $\left._{k}\right)$ and $\rho=\rho_{1} \cup \cdots \cup \rho_{k}$ and for all $1 \leq i \leq k$ we have $\operatorname{match}\left(v_{i}\right.$, Pat $\left._{i}\right)=\rho_{i}$. By T-MatchTup we have $\tau=\tau_{1} * \cdots * \tau_{k}$ and $\Gamma=\Gamma_{1} \cup \ldots \cup \Gamma_{k}$ and $\tau^{\prime}=\tau_{1}^{\prime} \cdots * \tau_{k}^{\prime}$ and for all $1 \leq i \leq k$ we have $\operatorname{match}\left(\tau_{i}, P a t_{i}\right)=\left(\Gamma_{i}, \tau_{i}^{\prime}\right)$.

Since $\vdash v: \tau^{\prime \prime}$, by T-Tup we have that $\tau^{\prime \prime}=\tau_{1}^{\prime \prime} * \cdots * \tau_{k}^{\prime \prime}$ and for all $1 \leq i \leq k$ we have $\vdash v_{i}: \tau_{i}^{\prime \prime}$. Since we're given that $\tau^{\prime \prime} \leq \tau$, by Lemma 19 we have $\tau_{i}^{\prime \prime} \leq \tau_{i}$ for all $1 \leq i \leq k$. Then by induction, for all $1 \leq i \leq k$ we have $\tau_{i}^{\prime \prime} \leq \tau_{i}^{\prime}$. Then by SuBTTup we have $\tau_{1}^{\prime \prime} * \cdots * \tau_{k}^{\prime \prime} \leq \tau_{1}^{\prime} * \ldots * \tau_{k}^{\prime}$, proving condition 1 . Also by induction, for all $1 \leq i \leq k$ we have $\operatorname{dom}\left(\Gamma_{i}\right)=\operatorname{dom}\left(\rho_{i}\right)$ and for each $\left(I_{0}, \tau_{0}\right) \in \Gamma_{i}$, there exists $\left(I_{0}, v_{0}\right) \in \rho_{i}$ such that $\vdash v_{0}: \tau_{0}^{\prime}$, where $\tau_{0}^{\prime} \leq \tau_{0}$. Therefore conditions 2 and 3 follow.

- Case E-MatchClass. Then $v=\left((\bar{\tau} C)\left\{\overline{V_{1}}=\overline{v_{1}}, \overline{V_{2}}=\overline{v_{2}}\right\}\right)$ and Pat has the form $\left(C^{\prime}\left\{\overline{V_{1}}=\overline{P_{a t}}\right)\right.$ and $C \leq C^{\prime}$ and $\rho=\bigcup \overline{\rho_{1}}$ and $\operatorname{match}\left(\overline{v_{1}}, \overline{P_{a t_{1}}}\right)=\overline{\rho_{1}}$. By TMatchClass we have $\tau=\left(\overline{\tau^{\prime}} C^{\prime \prime}\right)$ and $\tau^{\prime}=\left(\overline{\tau^{\prime}} C^{\prime}\right)$ and $\Gamma=\bigcup \overline{\Gamma_{1}}$ and $C^{\prime} \leq C^{\prime \prime}$ and repType $\left(\overline{\tau^{\prime}} C^{\prime}\right)=\left\{\overline{V_{1}}: \overline{\tau_{1}}\right\}$ and matchType $\left(\overline{\tau_{1}}, \overline{P_{\text {Pat }}}\right)=\left(\overline{\Gamma_{1}}, \overline{\tau_{1}^{\prime}}\right)$.

Since $\vdash v: \tau^{\prime \prime}$ and $v=\left((\bar{\tau} C)\left\{\overline{V_{1}}=\overline{v_{1}}, \overline{V_{2}}=\overline{v_{2}}\right\}\right)$, by T-REP we have that $\tau^{\prime \prime}=(\bar{\tau} C)$ and $\bullet \vdash(\bar{\tau} C)$ OK and and repType $(\bar{\tau} C)=\left\{\overline{V_{1}}: \overline{\tau_{1}^{\prime \prime}}, \overline{V_{2}}: \overline{\tau_{2}^{\prime \prime}}\right\}$ and $\vdash \overline{v_{1}}: \overline{\tau_{1}^{\prime \prime \prime}}$ and $\overline{\tau_{1}^{\prime \prime \prime}} \leq \overline{\tau_{1}^{\prime \prime}}$. Since $\tau^{\prime \prime} \leq \tau$, we have $(\bar{\tau} C) \leq\left(\overline{\tau^{\prime}} C^{\prime \prime}\right)$, so by Lemma 14 we have $\bar{\tau}=\overline{\tau^{\prime}}$. Since $C \leq C^{\prime}$ and $\bullet \vdash(\bar{\tau} C)$ OK, by Lemma 16 we have $(\bar{\tau} C) \leq\left(\bar{\tau} C^{\prime}\right)$, and since $\bar{\tau}=\overline{\tau^{\prime}}$, condition 1 is shown. By Lemma 36 we have $\overline{\tau_{1}^{\prime \prime}}=\overline{\tau_{1}}$. Therefore $\vdash \overline{v_{1}}: \overline{\tau_{1}^{\prime \prime \prime}}$ and $\overline{\tau_{1}^{\prime \prime \prime}} \leq \overline{\tau_{1}}$ and $\operatorname{match}\left(\overline{v_{1}}, \overline{P a t_{1}}\right)=\overline{\rho_{1}}$ and matchType $\left(\overline{\tau_{1}}, \overline{P_{1} t_{1}}\right)=\left(\overline{\Gamma_{1}}, \overline{\tau_{1}^{\prime}}\right)$, so by induction it follows that $\operatorname{dom}\left(\bigcup \overline{\Gamma_{1}}\right)=$ $\operatorname{dom}\left(\bigcup \overline{\rho_{1}}\right)$ and for each $\left(I_{0}, \tau_{0}\right) \in \bigcup \overline{\Gamma_{1}}$, there exists $\left(I_{0}, v_{0}\right) \in \bigcup \overline{\rho_{1}}$ such that $\vdash v_{0}: \tau_{0}^{\prime}$, where $\tau_{0}^{\prime} \leq \tau_{0}$. Therefore, conditions 2 and 3 are shown.

\section{A.3 Basic Lemmas}

These lemmas state some simple and intuitive properties about EmL's semantics. The proofs are all straightforward and can be found in the first author's dissertation [Millstein 2003].

\section{A.3.1 Type Well-formedness}

LEMma 11. If $\bar{\alpha} \vdash \tau$ OK, then all type variables in $\tau$ are in $\bar{\alpha}$.

\section{A.3.2 Subclassing and Subtyping}

Lemma 12. If $\tau \leq(\bar{\tau} C)$, then $\tau$ has the form $\left(\overline{\tau_{1}} C^{\prime}\right)$.

Lemma 13. If $(\bar{\tau} C) \leq \tau$, then $\tau$ has the form $\left(\overline{\tau_{1}} C^{\prime}\right)$.

Lemma 14. If $(\bar{\tau} C) \leq\left(\overline{\tau_{1}} C^{\prime}\right)$, then $\bar{\tau}=\overline{\tau_{1}}$. 
Lemma 15. If $(\bar{\tau} C) \leq\left(\overline{\tau_{1}} C^{\prime}\right)$ then $C \leq C^{\prime}$.

Lemma 16. If $C_{1} \leq C_{2}$ and $\bar{\alpha} \vdash\left(\bar{\tau} C_{1}\right)$ OK then (1) $\left(\bar{\tau} C_{1}\right) \leq\left(\bar{\tau} C_{2}\right)$; and (2) $\bar{\alpha} \vdash\left(\bar{\tau} C_{2}\right) O K$.

Lemma 17. If $\tau \leq \tau_{1} \rightarrow \tau_{2}$, then $\tau$ has the form $\tau_{1}^{\prime} \rightarrow \tau_{2}^{\prime}$, where $\tau_{1} \leq \tau_{1}^{\prime}$ and $\tau_{2}^{\prime} \leq \tau_{2}$

Lemma 18. If $\tau_{1} \rightarrow \tau_{2} \leq \tau$, then $\tau$ has the form $\tau_{1}^{\prime} \rightarrow \tau_{2}^{\prime}$.

Lemma 19. If $\tau \leq \tau_{1} * \cdots * \tau_{k}$, then $\tau$ has the form $\tau_{1}^{\prime} * \cdots * \tau_{k}^{\prime}$, where for all $1 \leq i \leq k$ we have $\tau_{i}^{\prime} \leq \tau_{i}$.

LEMMA 20. If $\tau_{1} * \cdots * \tau_{k} \leq \tau$, then $\tau$ has the form $\tau_{1}^{\prime} * \cdots * \tau_{k}^{\prime}$, where for all $1 \leq i \leq k$ we have $\tau_{i} \leq \tau_{i}^{\prime}$.

\section{A.3.3 Type Substitution}

Lemma 21. If $\tau \leq \tau^{\prime}$ and $|\bar{\alpha}|=|\bar{\tau}|$, then $[\bar{\alpha} \mapsto \bar{\tau}] \tau \leq[\bar{\alpha} \mapsto \bar{\tau}] \tau^{\prime}$.

Lemma 22. If $\Gamma ; \bar{\alpha} \vdash E: \tau$ and $|\bar{\alpha}|=|\bar{\tau}|$ and $\overline{\alpha_{0}} \vdash \bar{\tau} O K$, then $[\bar{\alpha} \mapsto \bar{\tau}] \Gamma ; \overline{\alpha_{0}} \vdash$ $[\bar{\alpha} \mapsto \bar{\tau}] E:[\bar{\alpha} \mapsto \bar{\tau}] \tau$.

Lemma 23. If repType $(C t)=\{\bar{V}: \bar{\tau}\}$ and $|\bar{\alpha}|=|\bar{\tau}|$, then repType $([\bar{\alpha} \mapsto$ $\bar{\tau}] C t)=[\bar{\alpha} \mapsto \bar{\tau}]\{\bar{V}: \bar{\tau}\}$.

Lemma 24. If matchType $(\tau$, Pat $)=\left(\Gamma, \tau^{\prime}\right)$ and $|\bar{\alpha}|=|\bar{\tau}|$, then matchType $([\bar{\alpha} \mapsto \bar{\tau}] \tau$, Pat $)=\left([\bar{\alpha} \mapsto \bar{\tau}] \Gamma,[\bar{\alpha} \mapsto \bar{\tau}] \tau^{\prime}\right)$.

A.3.4 Pattern Matching, Specificity, and Intersection

Lemma 25. If match $(v$, Pat $)=\rho$ and Pat $\leq$ Pat ${ }^{\prime}$, then there exists $\rho^{\prime}$ such that $\operatorname{match}\left(v, \operatorname{Pat}^{\prime}\right)=\rho^{\prime}$.

Lemma 26. If Pat $\leq$ Pat $^{\prime}$ and Pat $^{\prime} \leq$ Pat $^{\prime \prime}$ then Pat $\leq$ Pat".

Lemma 27. If Pat $\leq$ Pat then there exists some Pat $t_{0}$ such that Pat $\cap$ Pat ${ }^{\prime}=$ Pat $_{0}$.

Lemma 28. If Pat $\cap$ Pat $^{\prime}=$ Pat $_{0}$ then Pat $t_{0} \leq$ Pat and Pat $t_{0} \leq$ Pat $^{\prime}$.

Lemma 29. If owner $\left(M t\right.$, Pat $\left.^{\prime}\right)=C^{\prime}$ and owner $\left(M t\right.$, Pat $\left.^{\prime \prime}\right)=C^{\prime \prime}$ and Pat $\cap$ Pat ${ }^{\prime \prime}=$ Pat, then either $C^{\prime} \leq C^{\prime \prime}$ or $C^{\prime \prime} \leq C^{\prime}$.

Lemma 30. If $\vdash v: \tau$ and match $\left(v, P^{\prime} t^{\prime}\right)=\rho^{\prime}$ and match $\left(v, P a t^{\prime \prime}\right)=\rho^{\prime \prime}$ and matchType $\left(\tau^{\prime}, P a t^{\prime}\right)=\left(\Gamma^{\prime}, \tau_{0}^{\prime}\right)$ and matchType $\left(\tau^{\prime \prime}, P a t^{\prime \prime}\right)=\left(\Gamma^{\prime \prime}, \tau_{0}^{\prime \prime}\right)$, then there exists some Pat such that Pat $\cap$ Pat ${ }^{\prime \prime}=$ Pat.

Lemma 31. If $\operatorname{match}\left(v, P_{a t}\right)=\rho^{\prime}$ and $\operatorname{match}\left(v, P^{\prime \prime} t^{\prime \prime}\right)=\rho^{\prime \prime}$ and $\operatorname{Pat}^{\prime} \cap \operatorname{Pat}^{\prime \prime}=$ Pat, then there exists some $\rho$ such that match $(v$, Pat $)=\rho$.

A.3.5 Representations and Representation Types

Lemma 32. If $\Gamma ; \bar{\alpha} \vdash C t\left(\overline{E_{0}}\right)$ OK then there exist $\bar{V}$ and $\bar{E}$ such that $\operatorname{rep}\left(\operatorname{Ct}\left(\overline{E_{0}}\right)\right)=\{\bar{V}=\bar{E}\}$.

ACM Transactions on Programming Languages and Systems, Vol. 26, No. 5, September 2004. 
Lemma 33. If $\bar{\alpha} \vdash$ Ct $O K$ then there exist $\overline{V_{0}}$ and $\overline{\tau_{0}}$ such that repType $(C t)=$ $\left\{\overline{V_{0}}: \overline{\tau_{0}}\right\}$ and $\bar{\alpha} \vdash \overline{\tau_{0}} O K$.

Lemma 34. If $\operatorname{rep}(C t(\bar{E}))=\left\{\overline{V_{1}}=\overline{E_{1}}\right\}$ and repType $(C t)=\left\{\overline{V_{2}}: \overline{\tau_{2}}\right\}$ then $\overline{V_{1}}=\overline{V_{2}}$

Lemma 35. If $\Gamma_{0} ; \overline{\alpha_{0}} \vdash C t(\bar{E}) O K$ and $\operatorname{rep}(C t(\bar{E}))=\left\{\overline{V_{0}}=\overline{E_{0}}\right\}$ and repType $(C t)=\left\{\overline{V_{0}}: \overline{\tau_{0}}\right\}$, then $\Gamma_{0} ; \overline{\alpha_{0}} \vdash \overline{E_{0}}: \overline{\tau_{0}^{\prime}}$, for some $\overline{\tau_{0}^{\prime}}$ such that $\overline{\tau_{0}^{\prime}} \leq \overline{\tau_{0}}$.

Lemma 36. If $\bullet \vdash C t O K$ and $C t \leq C t^{\prime}$ then repType $(C t)$ has the form $\left\{\overline{V_{1}}\right.$ : $\left.\overline{\tau_{1}}, \overline{V_{2}}: \overline{\tau_{2}}\right\}$, where repType $\left(C t^{\prime}\right)=\left\{\overline{V_{1}}: \overline{\tau_{1}}\right\}$.

\section{A.3.6 Module Dependency Relation}

Lemma 37. If $\overline{S n} \vdash$ ITCTransUses and $C \leq C^{\prime}$, then $\overline{S n} \vdash$ $C^{\prime}$ ITCTransUses.

\section{ACKNOWLEDGMENTS}

Thanks to Jonathan Aldrich, Sorin Lerner, and Vass Litvinov for helpful comments on the paper.

\section{REFERENCES}

Ancona, D., Lagorio, G., AND ZuCCA, E. 2002. A formal framework for Java separate compilation. In Proceedings of the 2002 European Conference on Object-Oriented Programming, Lecture Notes in Computer Science, vol. 2374, Malaga, Spain (June). Springer-Verlag.

Ancona, D. And Zucca, E. 2001. True modules for Java-like languages. In J. L. Knudsen, Ed. ECOOP 2001-Object-Oriented Programming, Lecture Notes in Computer Science, vol. 2072. Springer.

Ancona, D. And Zucca, E. 2002. A calculus of module systems. J. Funct. Prog. 12, 2, 91-132 (March).

Arnold, K., Gosling, J., And Holmes, D. 2000. The Java Programming Language Third Edition. Addison-Wesley, Reading, MA, third edition.

BonNiot, D. 2002. Type-checking multi-methods in ML (a modular approach). In The Ninth International Workshop on Foundations of Object-Oriented Languages, FOOL 9, Portland, Oregon, USA (January).

BouRdoncle, F. ANd Merz, S. 1997. Type-checking higher-order polymorphic multi-methods. In Conference Record of POPL '97: The 24th ACM SIGPLAN-SIGACT Symposium on Principles of Programming Languages, 302-315, Paris, France, 15-17 (Jan).

BRACHA, G. AND CoOK, W. 1990. Mixin-based inheritance. In ECOOP/OOPSLA '90, 303-311.

CARDELli, L. 1988. A semantics of multiple inheritance. Information and Computation 76, 2/3, 138-164 (Feb).

CARDELli, L. 1997. Program fragments, linking, and modularization. In Conference Record of POPL '97: The 24th ACM SIGPLAN-SIGACT Symposium on Principles of Programming Languages, pages 266-277, Paris, France, 15-17 (Jan.).

CARDELli, L. and Wegner, P. 1985. On understanding types, data abstraction, and polymorphism. ACM Comput. Surv. 17, 4, 471-522 (Dec.).

Chambers, C. and Chen, W. 1999. Efficient multiple and predicate dispatching. In L. Meissner, Ed. In Proceeings of the 1999 ACM SIGPLAN Conference on Object-Oriented Programming, Systems, Languages \& Applications (OOPSLA'99), volume 34.10 of ACM Sigplan Notices, 238-255, N.Y., Nov. 1-5. ACM Press.

Chambers, C. and Leavens, G. T. 1995. Typechecking and modules for multimethods. ACM Trans. Prog. Lang. Sys. 17, 6, 805-843 (Nov.). 
Clifton, C., Leavens, G. T., Chambers, C., and Millstein, T. 2000. MultiJava: Modular open classes and symmetric multiple dispatch for Java. In OOPSLA 2000 Conference on Object-Oriented Programming, Systems, Languages, and Applications, Minneapolis, Minnesota, volume 35(10) of ACM SIGPLAN Notices, 130-145 (Oct.).

Соок, W. R. 1991. Object-oriented programming versus abstract data types. In J. W. de Bakker, W. P. de Roever, and G. Rozenberg, Eds. Foundations of Object-Oriented Languages, REX School / Workshop, Noordwijkerhout, The Netherlands, May/June, LNCS 489, 151-178. SpringerVerlag, New York, NY.

Drossopoulou, S., Eisenbach, S., and WragG, D. 1999. A fragment calculus-towards a model of separate compilation, linking and binary compatibility. In Logic in Computer Science, 147156.

DugGan, D. AND Sourelis, C. 1996. Mixin modules. In Proceedings of the 1996 ACM SIGPLAN International Conference on Functional Programming, 262-273, Philadelphia, Pennsylvania (May).

DugGan, D. and Techaubol, C.-C. 2001. Modular mixin-based inheritance for application frameworks. In Proceedings of the OOPSLA '01 conference on Object Oriented Programming Systems Languages and Applications, 223-240. ACM Press.

ERnst, M., Kaplan, C., And Chambers, C. 1998. Predicate dispatching: A unified theory of dispatch. In E. Jul, Ed. ECOOP '98-Object-Oriented Programming, Lecture Notes in Computer Science, vol. 1445, 186-211. Springer.

FindleR, R. B. ANd Flatt, M. 1998. Modular object-oriented programming with units and mixins. In Proceedings of the ACM SIGPLAN International Conference on Functional Programming (ICFP' 98), 34(1) of ACM SIGPLAN Notices, 94-104. ACM (June).

Fisher, K. AND REPPY, J. 1999. The design of a class mechanism for MOBY. In Proceedings of the ACM SIGPLAN'99 Conference on Programming Language Design and Implementation, 37-49, Atlanta, Georgia (May 1-4).

Fisher, K. AND RePpy, J. 2000. Extending Moby with inheritance-based subtyping. In 14th European Conference on Object-Oriented Programming, LNCS 1850, 83-107 (June).

Flatt, M. and Felleisen, M. 1998. Units: Cool modules for HOT languages. In Proceedings of the ACM SIGPLAN'98 Conference on Programming Language Design and Implementation (PLDI), 236-248, Montreal, Canada (17-19 June).

Flatt, M., Krishnamurthi, S., and Felleisen, M. 1998. Classes and mixins. In Conference Record of POPL 98: The 25TH ACM SIGPLAN-SIGACT Symposium on Principles of Programming Languages, San Diego, California, 171-183, New York, NY.

Fun, Y.-C. C. And Mishra, P. 1990. Type inference with subtypes. Theoretical Computer Science 73, 2, 155-175 (June).

Gamma, E., Helm, R., Johnson, R. E., And Vlissides, J. 1995. Design Patterns: Elements of Reusable Object-Oriented Software. Addison-Wesley, Massachusetts.

GARRIGUE, J. 2000. Code reuse through polymorphic variants. In Workshop on Foundations of Software Engineering, November.

Gosling, J., Joy, B., Steele, G., and Bracha, G. 2000. The Java Language Specification Second Edition. The Java Series. Addison-Wesley, Boston, Mass.

Hirschowitz, T. And Leroy, X. 2002. Mixin modules in a call-by-value setting. In D. Le Métayer, Ed, Programming Languages and Systems, ESOP'2002, volume 2305 of Lecture Notes in Computer Science, 6-20. Springer-Verlag.

Hoang, M. and Mitchell, J. C. 1995. Lower bounds on type inference with subtypes. In Conference Record of POPL '95: 22nd Annual ACM SIGPLAN-SIGACT Symposium on Principles of Programming Languages, San Francisco, Calif., 176-185, New York, NY (Jan.) ACM.

IgARAshi, A., Pierce, B. C., AND Wadler, P. 2001. Featherweight Java: A minimal core calculus for Java and GJ. ACM Transactions on Programming Languages and Systems 23, 3, 396-450, May.

Kahrs, S., Sannella, D., and Tarlecki, A. 1997. The definition of extended ML: A gentle introduction. Theoretical Computer Science 173, 2, 445-484, 28(Feb.).

Krishnamurthi, S., Felleisen, M., and Friedman, D. P. 1998. Synthesizing object-oriented and functional design to promote re-use. In E. Jul, Ed. ECOOP'98-Object-Oriented Programming, 12th European Conference, Brussels, Belgium, Lecture Notes in Computer Science, vol. 1445, 91-113. Springer-Verlag (July). 
MacQueEn, D. 1984. Modules for standard ML. In Conference Record of the 1984 ACM Symposium on Lisp and Functional Programming, 198-207. ACM (Aug.).

McDirmid, S., Flatt, M., ANd Hsieh, W. C. 2001. Jiazzi: new-age components for old-fashioned java. In Proceedings of the OOPSLA '01 conference on Object Oriented Programming Systems Languages and Applications, 211-222. ACM Press.

Millstein, T. 2003. Reconciling Software Extensibility with Modular Program Reasoning. Ph.D. dissertation, Department of Computer Science \& Engineering, University of Washington.

Millstein, T., Bleckner, C., And Chambers, C. 2002. Modular typechecking for hierarchically extensible datatypes and functions. In Proceedings of the ACM SIGPLAN International Conference on Functional Programming (ICFP '02), volume 37(9) of ACM SIGPLAN Notices, 110-122, New York, NY (Sept.) ACM.

Millstein, T. AND Chambers, C. 1999. Modular statically typed multimethods. In R. Guerraoui, editor, ECOOP '99 - Object-Oriented Programming 13th European Conference, Lisbon Portugal, Lecture Notes in Computer Science, vol. 1628, 279-303. Springer-Verlag, New York, NY (June).

Millstein, T. ANd Chambers, C. 2002. Modular statically typed multimethods. Information and Computation 175, 1, 76-118 (May).

Milner, R., Tofte, M., Harper, R., and MacQueen, D. 1997. The Definition of Standard ML (Revised). The MIT Press.

Nelson, G. 1991. Systems Programming with Modula-3. Prentice Hall.

NoRdLANDER, J. 1999. Pragmatic subtyping in polymorphic languages. In Proceedings of the ACM SIGPLAN International Conference on Functional Programming (ICFP '98), 34, 1, 216-227.

Odersky, M. AND WadLER, P. 1997. Pizza into Java: Translating theory into practice. In Conference Record of POPL '97: The 24th ACM SIGPLAN-SIGACT Symposium on Principles of Programming Languages, 146-159, Paris, France, 15-17 (Jan.).

Pierce, B. C. And Turner, D. N. 2000. Local type inference. ACM Trans. Prog. Lang. Syst. 22, 1, 1-44 (Jan.).

RÉmy, D. ANd Voulllon, J. 1998. Objective ML: An effective object-oriented extension of ML. Theory and Practice of Object Systems 4, 1, 27-52.

Reppy, J. AND RIECKe, J. 1996. Simple objects for Standard ML. In Proceedings of the ACM SIGPLAN '96 Conference on Programming Language Design and Implementation, 171-180, Philadelphia, Pennsylvania, 21-24 (May).

REYNOLDS, J. C. 1978. User defined types and procedural data structures as complementary approaches to data abstraction. In D. Gries, editor, Programming Methodology, A Collection of Articles by IFIP WG2.3, 309-317. Springer-Verlag, New York, NY.

Shalit, A. 1997. The Dylan Reference Manual: The Definitive Guide to the New Object-Oriented Dynamic Language. Addison-Wesley, Reading, Mass.

WADLER, P. 1990. Linear types can change the world! In M. Broy and C. Jones, editors, IFIP TC 2 Working Conference on Programming Concepts and Methods, Sea of Galilee, Israel, 7-359. North Holland (Apr.).

Wright, A. K. ANd Felleisen, M. 1994. A syntactic approach to type soundness. Information and Computation 115, 1, 38-94, 15 (Nov.).

Zenger, M., AND Odersky, M. 2001. Extensible algebraic datatypes with defaults. In Proceedings of the 2001 ACM SIGPLAN International Conference on Functional Programming. ACM (September) 3-5.

Received August 2002; revised September 2003; accepted February 2004 